\title{
WestVirginiaUniversity
}

THE RESEARCH REPOSITORY @ WVU

Graduate Theses, Dissertations, and Problem Reports

2011

\section{Intelligent reservoir modeling of Lower Huron Shale}

Ognjen Grujic

West Virginia University

Follow this and additional works at: https://researchrepository.wvu.edu/etd

\section{Recommended Citation}

Grujic, Ognjen, "Intelligent reservoir modeling of Lower Huron Shale" (2011). Graduate Theses,

Dissertations, and Problem Reports. 4725.

https://researchrepository.wvu.edu/etd/4725

This Thesis is protected by copyright and/or related rights. It has been brought to you by the The Research Repository @ WVU with permission from the rights-holder(s). You are free to use this Thesis in any way that is permitted by the copyright and related rights legislation that applies to your use. For other uses you must obtain permission from the rights-holder(s) directly, unless additional rights are indicated by a Creative Commons license in the record and/ or on the work itself. This Thesis has been accepted for inclusion in WVU Graduate Theses, Dissertations, and Problem Reports collection by an authorized administrator of The Research Repository @ WVU. For more information, please contact researchrepository@mail.wvu.edu. 


\title{
INTELLIGENT RESERVOIR MODELING OF LOWER HURON SHALE
}

\author{
Ognjen Grujić
}

\author{
Thesis submitted to the \\ College of Engineering and Mineral Resources \\ at West Virginia University \\ in partial fulfillment of the requirements \\ for the degree of \\ Master Of Science \\ In \\ Petroleum And Natural Gas Engineering \\ Shahab D. Mohaghegh, PhD., Chair. \\ Samuel Ameri, MS. \\ Grant Bromhal, PhD.
}

Morgantown, West Virginia

2011

Keywords: Lower Huron Shale, Intelligent Reservoir Modeling, Artificial Intelligence, Production Data Analysis, Reservoir Simulation 


\section{ABSTRACT \\ Intelligent Reservoir Modeling of Lower Huron Shale}

\section{Ognjen Grujić}

The work presented in this thesis is an intelligent reservoir modeling and analysis of Lower Huron Shale in eastern Kentucky. Methodology used for this analysis is a recently developed Top Down Intelligent Reservoir Modeling, that couples artificial intelligence and data mining techniques with conventional reservoir engineering methods. A total of 77 wells completed in Lower Huron Shale in eastern Kentucky were used in this study. Well production data was obtained from the company operating the wells, while completion reports and well logs were downloaded from publicly available database at Kentucky Geological Surveys website. The downloaded well logs were digitized, and detailed geological interpretation of the studied area was performed. More information about the reservoir was acquired through decline and type curve analyses and single well history matching. Single well history matching was performed with publicly available shale and tight gas reservoir simulator. All of the acquired data was used in the development of spatiotemporal dataset that was further analyzed with state of the art in artificial intelligence and data mining (fuzzy pattern recognition, artificial neural networks). Finally, reservoir was divided into zones of different relative reservoir quality and full field artificial intelligence empowered predictive model of the reservoir was developed and verified. 
To my parents, Srećko and Nada Grujić 


\section{ACKNOWLEDGEMENTS}

First of all I would like to thank my family for all the love and support they have provided me with throughout the years.

Secondly I would like to thank my advisor professor Shahab D. Mohaghegh for his endless guidance, patience, help, and support which he has been giving me for the last two years. I will always remember and appreciate what he has done for me. I would also like to thank my committee members: Professor Samuel Ameri, and Dr. Grant Bromhal for accepting to be in my committee, for their support and valuable suggestions.

I would like to thank Mr. and Mrs. Jikich, who have been helping me ever since the day when I decided to pursue my MS degree at WVU. During my stay in Morgantown they were like a second family to me. I will always keep a special place in my heart for them. I would also like to use this opportunity to thank my professors Dr. Dusan Danilovic and Dr. Vesna Karovic Maricic from the University of Belgrade for all their help, support and friendship.

For the last two years I was very lucky to have worked with such wonderful people at PEARL ${ }^{1}$. Specifically I would like to thank: Mr. Yorgi Gomez, Mr. Camilo Calderon, Ms. Vida Gholami, Mrs. Yasaman Khazaeni, Mr. Abdullahi Yusuf, Ms. Shohreh Amini, Mrs Najmeh Borouzee, Mr. Ali Naser, Mrs. Qin He, Mr. Ike Olunokuokua, Mr. Alireza Sharakhami, Mr. Alireza Hagighat, Mr. Eduardo Delgado, and Mrs. Soodabeh Esmaili for all their friendship, help and great moments we had while working together. I am very thankful to my friends and colleagues at Intelligent Solutions Inc. Mr. Razi Gaskari and Mr. Mohammad Maysami, I will always remember with great pleasure the time spent working with them. I would also like to use this opportunity to thank Mr. Chris Zinn and EQT Company for providing me with the production data and for being very helpful and patient in answering all my questions. Also I would like to thank DOE/NETL for financially supporting my research, the outcome of which is exactly this thesis.

\footnotetext{
${ }^{1}$ Petroleum Engineering and Analytics Laboratory at WVU
} 
Many thanks to my friends Ms. Liliana Acosta Santacruz, Mr. Alexandar Filipovic, Ms. Ivana Vukotic, Mr. Francisco de Borja Caballero De La Cuadra, Mr. Alejandro Posada and his fiancée Isabella, for their endless friendship and support.

I must use this opportunity to thank Ms Jovana Cirkovic and Mr. Milos Lomovic for their true friendship and all the moments spent talking to me when I was missing home or needed a piece of advice. I feel truly blessed for having them as my friends.

Special thanks to Dr. Vladislav Kecojevic for his true friendship and support. For always being there for me with the best advices and support that anyone could give.

Finally I would like to thank Miss Karla Vargas for her endless love, compassion, friendship, support and assistance with reviewing this manuscript. 


\section{CONTENTS}

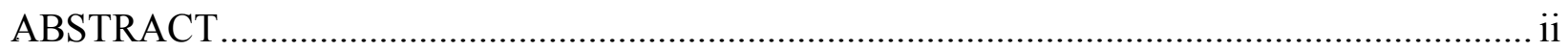

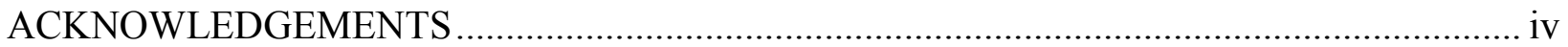

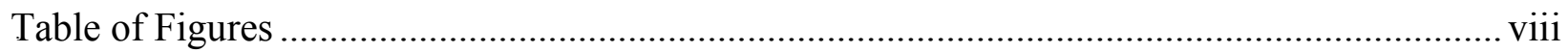

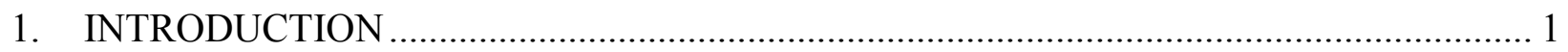

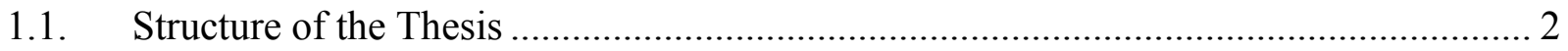

2. LITERATURE REVIEW AND THEORETICAL FRAMEWORK ...................................... 4

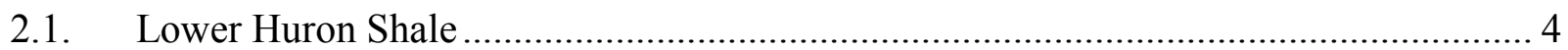

2.2. Artificial Intelligence ………………………................................................. 10

2.2.1. Biological Neural Networks .......................................................................... 11

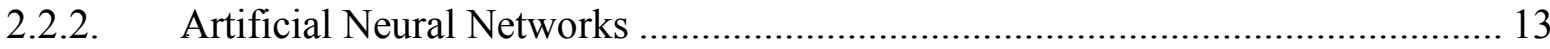

2.2.3. Back-propagation Neural Network (BACKPROP) ………................................ 17

3. METHODOLOGY OF THE TOP DOWN INTELLIGENT RESERVOIR MODELING... 19

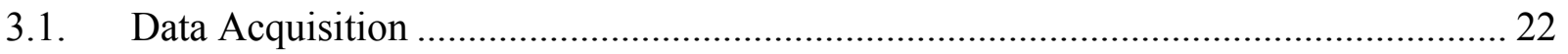

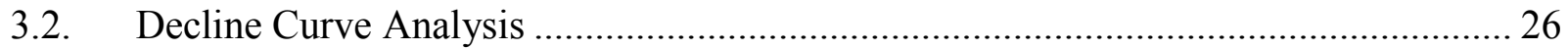

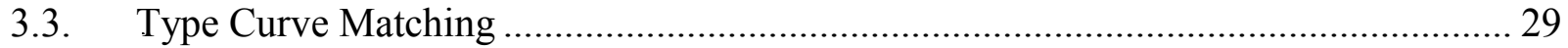

3.4. Single Well Numerical Simulation ......................................................................... 32

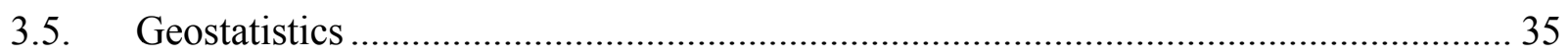

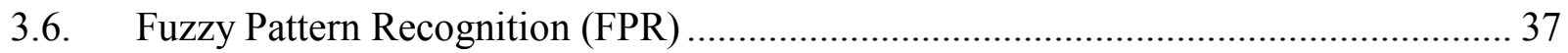

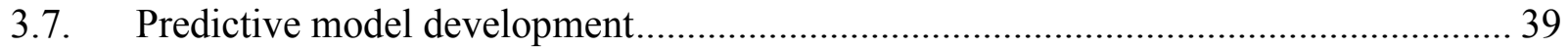




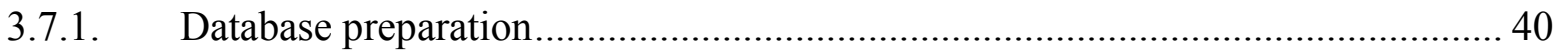

3.7.2. Key Performance Indicators Analysis (KPI Analysis) . ................................ 40

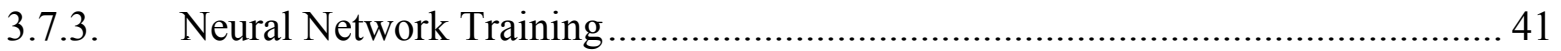

3.8. Model Implementation and Verification............................................................ 43

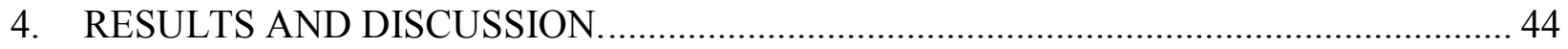

4.1. Fuzzy Pattern Recognition (FPR) Analysis .................................................. 44

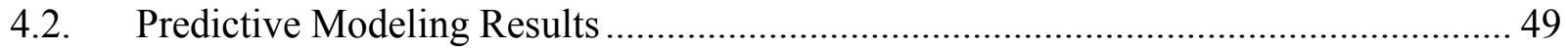

4.3. Predictive Modeling - Sensitivity Analysis.................................................... 55

5. CONCLUDING REMARKS \& FUTURE WORK................................................... 58

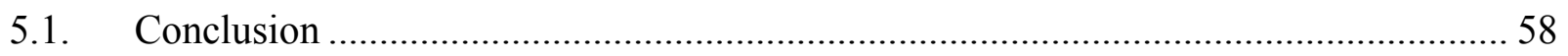

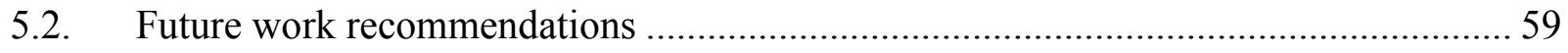

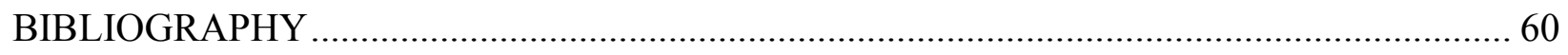

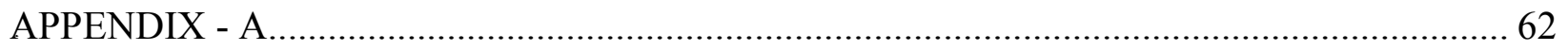

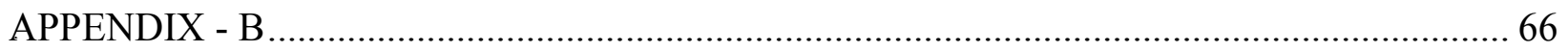




\section{Table of Figures}

Figure 1. Stratigraphic correlation of the Devonian shales. (Adopted from [11]) ......................... 5

Figure 2 Big Sandy Gas field (blue). (Modified from [11]) ……............................................... 6

Figure 3 Results of Kubik's (1993) research (Modified from [11]) …………............................ 7

Figure 4 Interval isolation results of the Kubik's study (Modified form [11]) …………................ 8

Figure 5 Well log response of the Lower Huron Shale. ......................................................... 10

Figure 6 An example of a neural cell, neuron (Adopted from [14]) .......................................... 12

Figure 7 Schematic representation of artificial neuron (Adopted from [8]) ……....................... 14

Figure 8 Most commonly used activation functions (Adopted from [8]) ………....................... 14

Figure 9 Layered structure of the Artificial Neural Networks (ANN) (Adopted from [14]) ….... 15

Figure 10 Supervised neural network training scheme ............................................................ 16

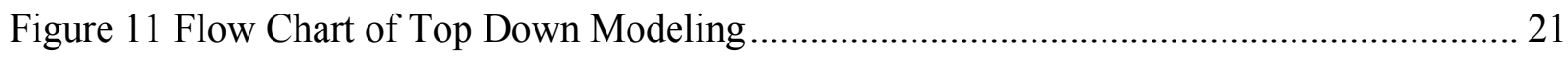

Figure 12 Gamma Ray (left) and Density (right), response distribution throughout the study area

Figure 13 Typical well log response of the Lower Huron shale in the study area. (from10])...... 24

Figure 14 East West Cross Section of the Study area (Lower Huron Shale is painted Brown) ... 25

Figure 15 Cartesian plot of all three cases (types) of Arp's decline curve equation...................... 28

Figure 16 Semi-Log plot of all three cases (types) of Arp's decline curve equation ..................... 28

Figure 17 Example of type curve matching procedure (adopted from IMAGINE software)........ 31

Figure 18 Flow Chart of Decline - Type curve analysis for a single well. ................................. 32 
Figure 19 Representation of the most probable natural fracture network within the Lower Huron Shale in eastern Kentucky (generated with Fracgen). ....................................................... 34

Figure 20 Example of a history matched production data with the Fracgen/Nfflow simulator. .. 35

Figure 21 Geostatistically produced map of formation depth (left) and formation thickness (right) 36

Figure 22 Spatial distribution maps of Log Porosity (left) and Formation permeability from type

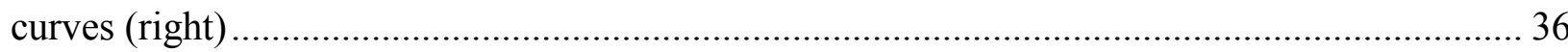

Figure 23 Detailed explanation of Fuzzy Pattern Recognition Methodology ........................... 38

Figure 24 Example of the KPI analysis performed in this study ........................................ 41

Figure 25 Neural Network selection of inputs (left), and example of training (right)............... 42

Figure 26 Example of database parsing for neural network training ................................... 42

Figure 27 Fuzzy Pattern Recognition Analysis of First 3 Months of Production...................... 45

Figure 28 Fuzzy Pattern Recognition Analysis of First 6 months of production ...................... 45

Figure 29 Fuzzy Pattern Recognition Analysis of the first year cumulative production............. 46

Figure 30 Fuzzy Pattern Recognition Analysis of First 3 years of production......................... 46

Figure 31 Fuzzy Pattern Recognition Analysis of First 5 Years of Cumulative production ........ 47

Figure 32 Fuzzy Pattern Recognition Analysis of First 10 Years of Cumulative production ...... 47

Figure 33 Fuzzy Pattern Recognition Analysis of Cumulative Gas production ........................ 48

Figure 34 Fuzzy Pattern Recognition Analysis of Total Gas Recovery (with existing wells) ..... 48

Figure 35 Neural Network Training Results (Y Axis - Real Data, X Axis -Neural Network

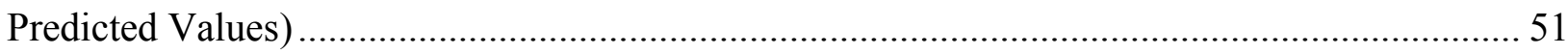

Figure 36 Real field production rate vs Top Down predictive models field production rate ....... 51

Figure 37 Example of Top-Down predictive model results for a single well.......................... 52 
Figure 38 Example of Top-Down predictive model results for a single well .......................... 52

Figure 39 Example of Top-Down predictive model results for a single well........................... 53

Figure 40 Example of Top-Down predictive model results for a single well.......................... 53

Figure 41 Example of Top-Down predictive model results for a single well........................... 54

Figure 42 Example of Top-Down predictive model results for a single well......................... 54

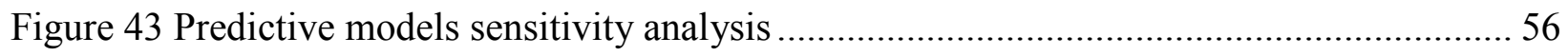

Figure 44 Predictive models sensitivity analysis .......................................................... 56

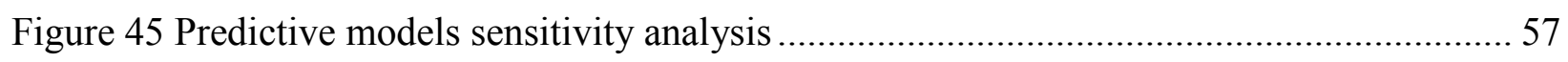

Figure 46 WVU - FGI Fracture Set Selection Screen....................................................... 63

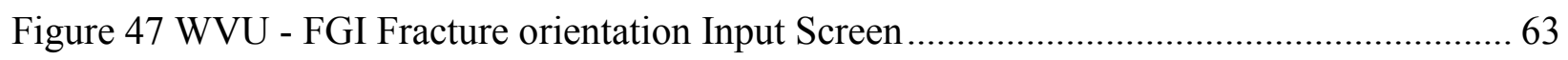

Figure 48 WVU - FGI Fracture properties - Input Screen .................................................. 64

Figure 49 WVU - FGI Fracture Network Visualization Screen ........................................... 64

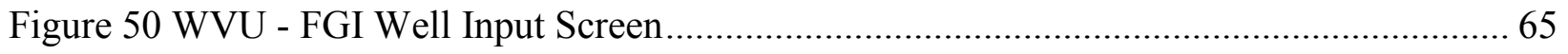




\section{INTRODUCTION}

In this thesis Top Down Intelligent Reservoir Modeling of the Lower Huron shale in eastern Kentucky was performed. Top Down Intelligent Reservoir Modeling is a methodology that utilizes artificial neural networks in deducting complex patterns of reservoir productivity indices and producing highly accurate full field predictive models.

All of the wells used in this study were completed in Lower Huron shale in eastern Kentucky and are a part of the well known Big Sandy gas field. Work presented here is one of the first Top Down Intelligent Reservoir Modeling studies performed on Devonian age shale gas reservoir in Appalachian basin.

As a part of this work Geographix Discovery geological model of the studied area was developed. 65 out of 77 well logs were digitized and interpreted, along with the accompanying completion reports. This study also included detailed literature review of the Lower Huron shale and shale gas reservoirs in general. Since emphasis of the applied methodology is on utilization of the artificial neural networks, significant amount of time was spent on the familiarization with such concept and previous applications of this technology to similar problems.

Most of the studied wells have production profiles lasting for more than twenty years. Porosity and thickness data was acquired from the available well logs, while additional data was acquired through Cox type curve matching procedure and single well history matching with Fracgen/Nfflow simulator package. As a part of this work a small contribution to the Fracgen/Nfflow simulation methodology was provided through the development of a custom build interface for Fracgen software. Computer application developed in this study significantly 
reduces the time needed for fracture network generation and overall reservoir simulation with Fracgen/Nfflow package.

Top Down Intelligent Reservoir Modeling methodology started by performing conventional reservoir engineering analyses on individual well basis, such as decline curve analysis, and type curve matching. Statistical techniques along with the information generated from the reservoir engineering analyses yielded to an extensive spatiotemporal database of reservoir behavior. Developed database was further used for the development of a cohesive model of the field by using fuzzy pattern recognition and artificial neural network based predictive model.

The predictive model developed in this study was verified vs. the existing production history, and also by performing predictions of future well performance of the existing wells. Based on the Fuzzy pattern recognition analysis, identification of the "sweet spots" in the reservoir was performed, and future infill drilling locations were proposed.

\subsection{Structure of the Thesis}

This thesis is organized into five chapters.

- Chapter 1 is the Introduction chapter which provides summary of the presented work and structure of this thesis.

- Chapter 2 is Literature Review and Theoretical framework where more information about the Lower Huron shale in eastern Kentucky is presented. The focus of this chapter is on the Lower Huron shale in eastern Kentucky because wells analyzed in this study were all completed in this formation. The second part of this chapter focuses on the basic concepts and theory behind the artificial neural networks, since the objective of the performed 
study was the development of full field reservoir model empowered by artificial intelligence.

- Chapter 3 - This chapter gives detailed description of the Top Down Intelligent Reservoir Methodology that was used in this study.

- Chapter 4 is reserved for the results obtained from Top Down Intelligent Reservoir Modeling. This chapter is focused on the fuzzy pattern recognition results in identification of the reservoirs "sweet spots" and predictive models results which are presented in the second section of this chapter.

- Chapter 5 is the Concluding chapter in which concluding remarks about the study are presented along with the proposed future actions. 


\section{LITERATURE REVIEW AND THEORETICAL FRAMEWORK}

\subsection{Lower Huron Shale}

It has been nearly 200 years since the first wells completed in Devonian age black shales started producing natural gas. What first began as accidental discovery, while drilling for other more prolific plays, nowadays represents the most important source of natural gas. The very first wells, completed in the Marcellus shale near New York in the 1920's had production in the amount that was high enough only to power street lamps of nearby towns [11]. "Low Productivity" of these reservoirs made them completely unattractive to the gas companies at the time, which moved their focus on to the other more productive reservoirs.

However, the situation was not the same with all the shale gas plays in the Appalachian basin. In 1921, accidental discovery of highly productive Devonian shale sequence well (over 1000 mscf/day) while drilling for deeper more prolific Coniferous reservoirs initiated the development of eastern Kentucky's Devonian shale.

This well belonged to what's later going to be called the Big Sandy gas field. Most of the production from the wells completed in the Big Sandy comes from Lower Huron member of the Ohio shale. Stratigraphic correlation of Devonian age rocks from eastern Kentucky through West Virginia is given in Figure 1. 


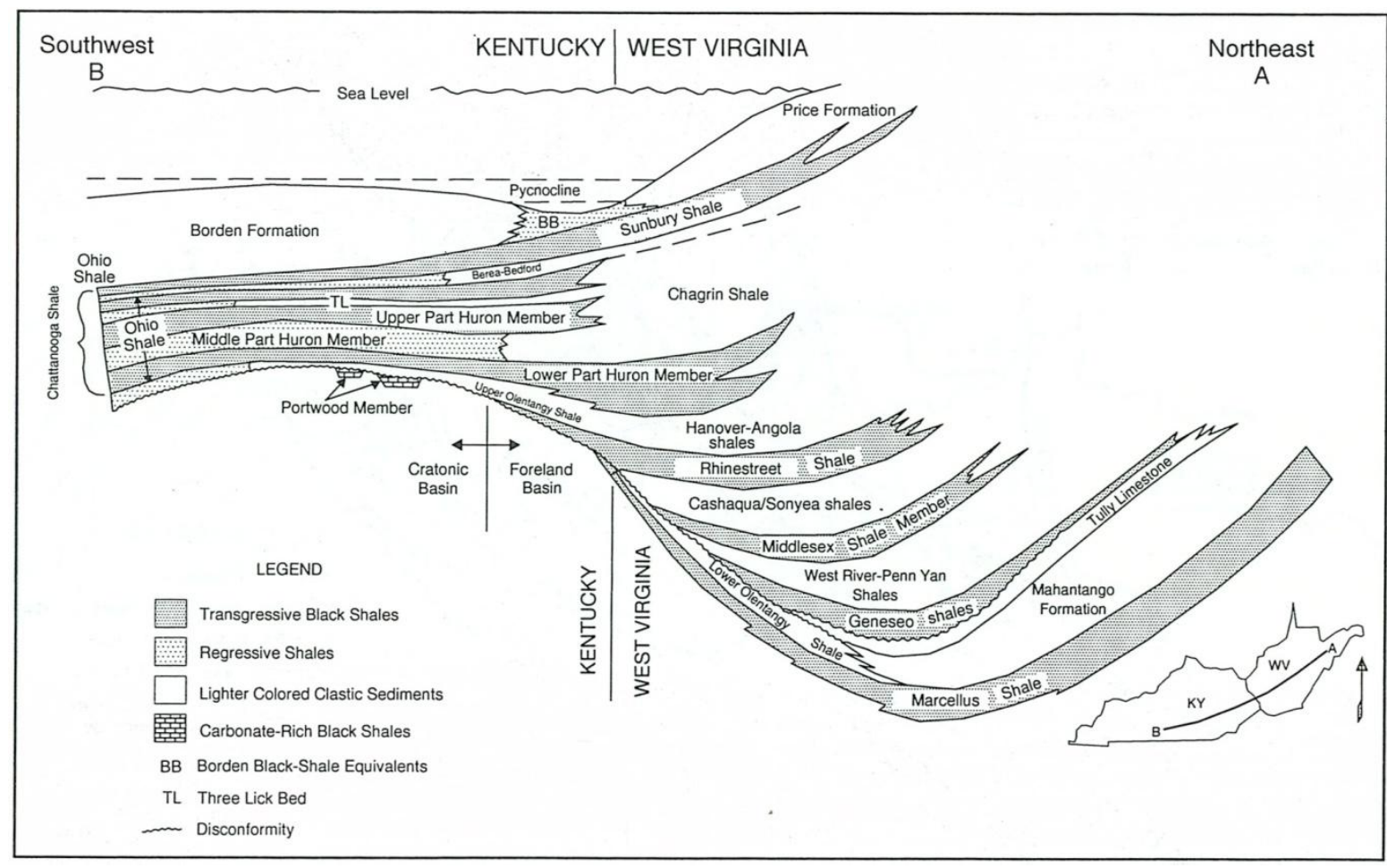

Figure 1. Stratigraphic correlation of the Devonian shales. (Adopted from [11]).

Right after the discovery of the first well, rapid development of the Big Sandy gas field began.

Development peak was achieved in 1940's when the number of wells completed in the Big Sandy was over 1,400 [11]. Even at that time it was believed that such high production is possible only thanks to the intensive natural fracture occurrences in the reservoir. Therefore, main stimulation method used on this field in order to create more contact with the natural fractures was firing large amounts of nitroglycerin (4,000-8,000 pounds) at the target zone. This method required very long cleanup times; however the results achieved were very impressive. This type of stimulation made completely non productive wells, highly productive [11]. Later in the 1960's when the usage of hydraulic fracturing as a standard completion method commenced, the development of the Big Sandy started to enter second development peak of its production history. Second peak of development was achieved in the 1980's when the number of drilled wells was almost the same as the number of wells drilled during the first development peak of the 1940's (approx. 1,400). 
Even though such intensive drilling was performed on this reservoir, very little scientific information was being collected and published. This continued until the Eastern Shales Gas Project (ESGP) was conducted by the DOE, when more information about fracture occurrences, thermal maturity, and stimulation treatments were gathered. ESGP studies found out that the most productive intervals were at the same time the most organically rich, and also highly radioactive. Another thing discovered, was that the zones of the reservoir that were to the east had much higher thermal maturity than the western portions of the reservoir. This was due to somewhat higher temperatures in the eastern portion caused by higher depth. More thermally mature zones were found to produce only dry gas, while western zones had indications of wet gas [11].

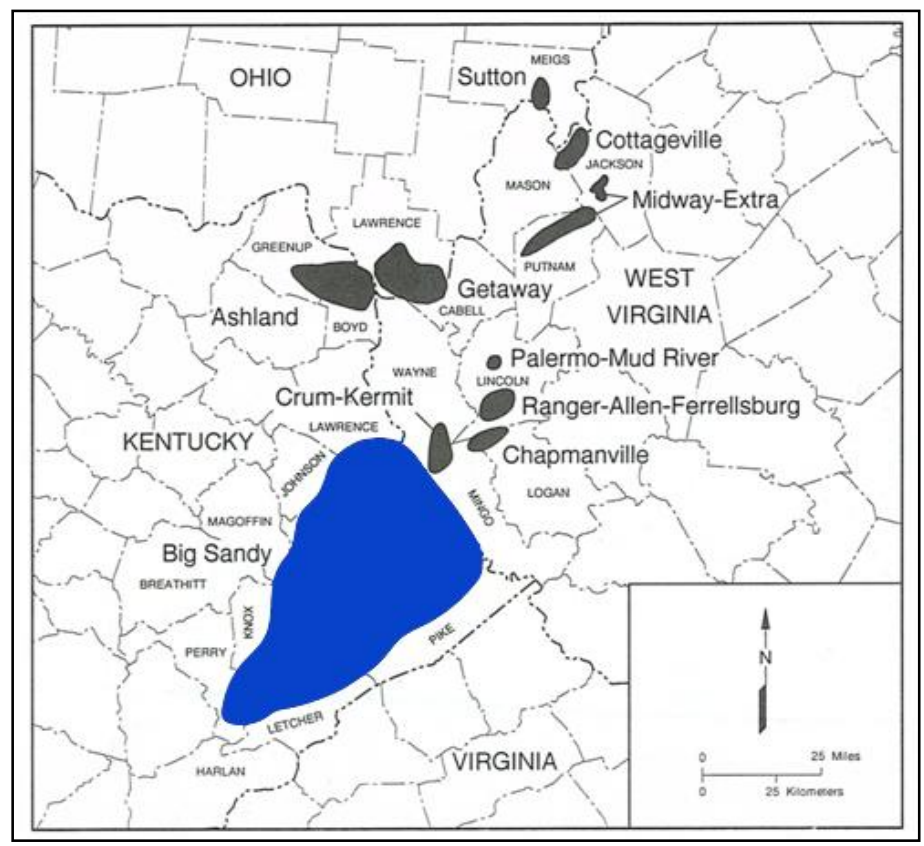

Figure 2 Big Sandy Gas field (blue). (Modified from [11])

Only one study, that is commonly quoted in all literature dealing with Lower Huron shale, conducted by Kubik in 1993 [4] for the Gas Research Institute (GRI) dealt with the natural fracture occurrences in the Big Sandy gas field. Kubik's study divided eastern Kentucky's black 
shale sequence into 7 different units of different natural fracture occurrences, and the amount of organic carbon (TOC). Results of Kubik's study can best be summarized with the following figure (Figure 3).

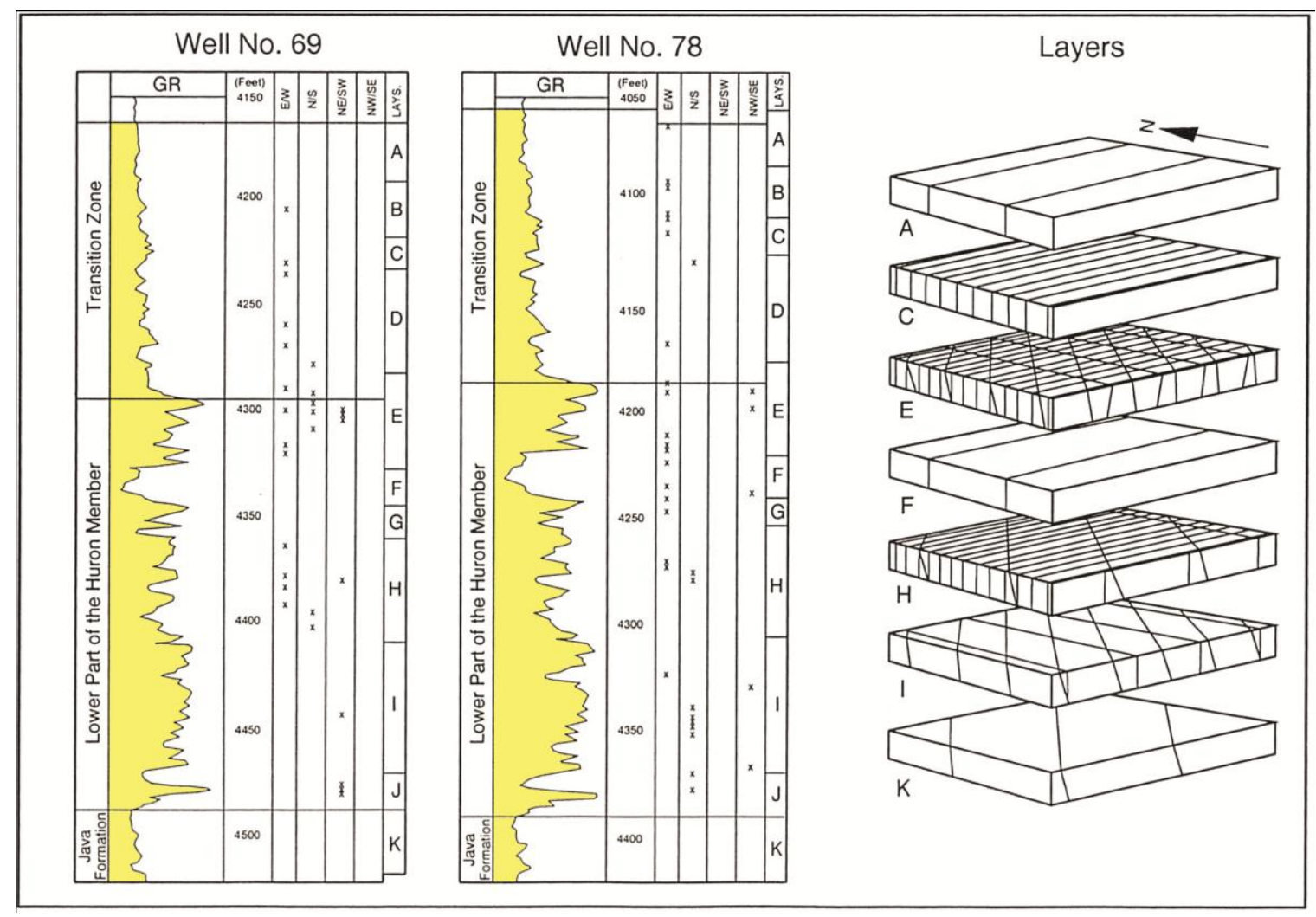

Figure 3 Results of Kubik's (1993) research (Modified from [11])

Kubik's study also performed isolation tests on the studied wells in order to get more insight into most productive intervals. (Figure 4) 


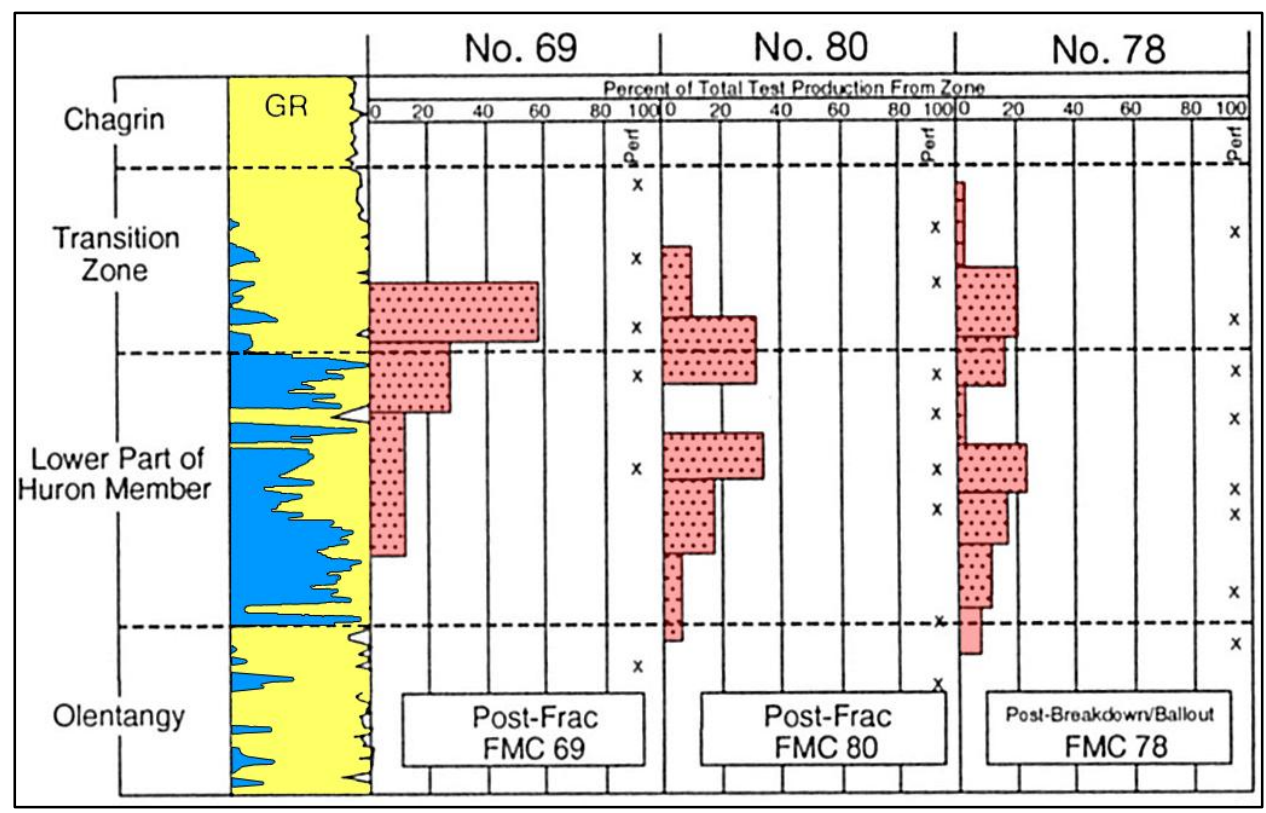

Figure 4 Interval isolation results of the Kubik's study (Modified form [11])

Figure 4 shows an interesting fact that even though Lower Huron shale is the most organically rich zone of the Devonian - Ohio shale sequence, the most productive layer is actually organically poor zone, the so called "Transition Zone" which belongs to the Middle Huron sequence. Kubik explained, that this is due to the fact that the "Transition Zone" is more intensively fractured than the Lower Huron zone [4], therefore gas was able to migrate from the Lower Huron shale into the upper more fractured and therefore more saturated and productive "Transition Zone" (secondary migration).

Later study conducted by Ning [9] on cores recovered from the Big Sandy's Lower Huron interval, measured permeabilities of both matrix and natural fractures. Ning's findings showed matrix permeability of the Lower Huron Shale to be in the range of $10^{-7}$ to the $10^{-9} \mathrm{md}$, and natural fracture permeabilities to be in the range of $10-300 \mathrm{md}$ (most extremely 2,500 $\mathrm{md}$ ). Ning's study confirmed that natural fracture occurrences provide necessary conduit for gas, and 
therefore make gas production possible from such reservoir. Reservoir properties of the Lower Huron shale in eastern Kentucky are summarized in a table below.

Table 1 Summarized reservoir properties of the Big Sandy gas field (from [11])

\begin{tabular}{||c|c|c|c||}
\hline \multirow{3}{*}{ Porosity, $\varnothing$} & \multirow{2}{|c|}{ Property } & Value \\
\hline \multirow{3}{*}{ Core Measurements } & & Minimum & $0.40 \%$ \\
\cline { 3 - 4 } & & Maximum & $8.60 \%$ \\
\cline { 3 - 4 } & \multirow{2}{*}{ Log Measurement } & Average & $4.30 \%$ \\
\cline { 3 - 4 } & & Maximum & $5 \%$ \\
\hline \multirow{2}{*}{ Matrix Permeability, md } & \multirow{2}{*}{ Core Measurements } & $15 \%$ \\
\cline { 3 - 4 } Fracture Permeability, md & \multirow{2}{*}{ Core Measurements } & Minimum & $10^{-9}$ \\
\cline { 3 - 4 } & & Maximum & $10^{-7}$ \\
\cline { 3 - 4 } & & Maximum & 300 \\
\hline \hline
\end{tabular}

On well logs Lower Huron Shale is indicated by high gamma ray readings ${ }^{2}$, usually sudden GR increases, accompanied with sudden decreases in density readings. Both occurrences are a consequence of the high amount of radioactive particles contained within the Lower Huron Shale. Example of a typical well log response of the Lower Huron shale is given in Figure 5.

${ }^{2}$ High radioactivity is associated with high organic content, due to the depositional affinity of radioactive elements $(U, T h, K)$ to kerogen (organic matter in shales). Therefore, well log readings recorded in highly organically rich zones manifest in very high GR readings. 


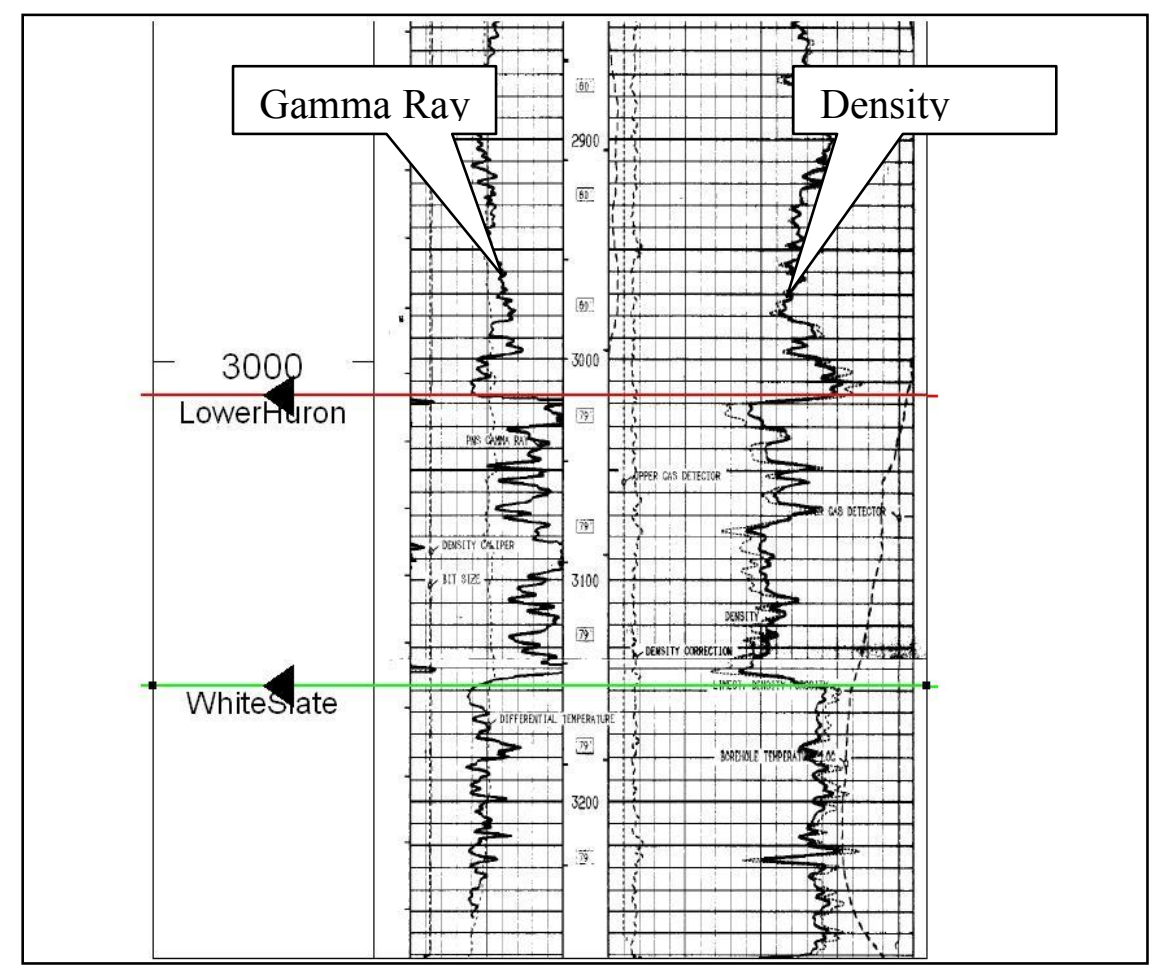

Figure 5 Well log response of the Lower Huron Shale.

As it was said before typical completion method in the Big Sandy gas field for the last 40 years was hydraulic fracturing. The most typical frac jobs in the field were conducted by injecting 14,000 gallons of water (typically) and approximately 50,000 to 60,000 pounds of sand [11].

\subsection{Artificial Intelligence}

The development of artificial intelligence came from the need of creating computer based systems that would be able to automatically learn, think and interact with the environment independently, or in other words without human involvement or predefined rules that they are programmed to follow. Naturally ideas for the development of such systems came from nature, since the concept of intelligence currently known to us comes from highly developed biological creatures to which we, humans also belong. Therefore, most modern solutions in artificial intelligence domain were developed based on the mechanisms found in biological systems. 
Most widely accepted concepts in artificial intelligence are artificial neural networks and genetic algorithms. The methodology used in this study utilized artificial neural networks in the development of full field reservoir models capable of making accurate production predictions. Therefore, significant amount of space in this chapter is given exactly to Artificial Neural Networks (ANN). But before any explanation is given about the concepts behind ANN's, it is important to show how do real intelligent biological systems work, what are the basic mechanism of biological intelligence, and how they inspired the development of Artificial Neural Networks.

\subsubsection{Biological Neural Networks}

All living organisms are very complex systems constituted of very large number of small units, cells. Within the organisms, cells of different types are organized into larger units (organs) that take different functions in maintaining stable living condition of an organism. In a more generalized observation, living organisms are constituted of complex systems of organs. In human organism, for example, modern science distinguishes five types of organ systems: digestive system, respiratory system, circulatory system, musculoskeletal system, and nervous system.

The nervous system consists of a large number of interconnected cells called neural cells or simply neurons. In a nutshell, the main function of neural system is controlling the organism, both in terms of controlling basic metabolic functions, and organism's interaction with the environment by extracting information from the environment, processing it and producing appropriate reactions. [14]

In Figure 6 sketch of biological neuron is given. 


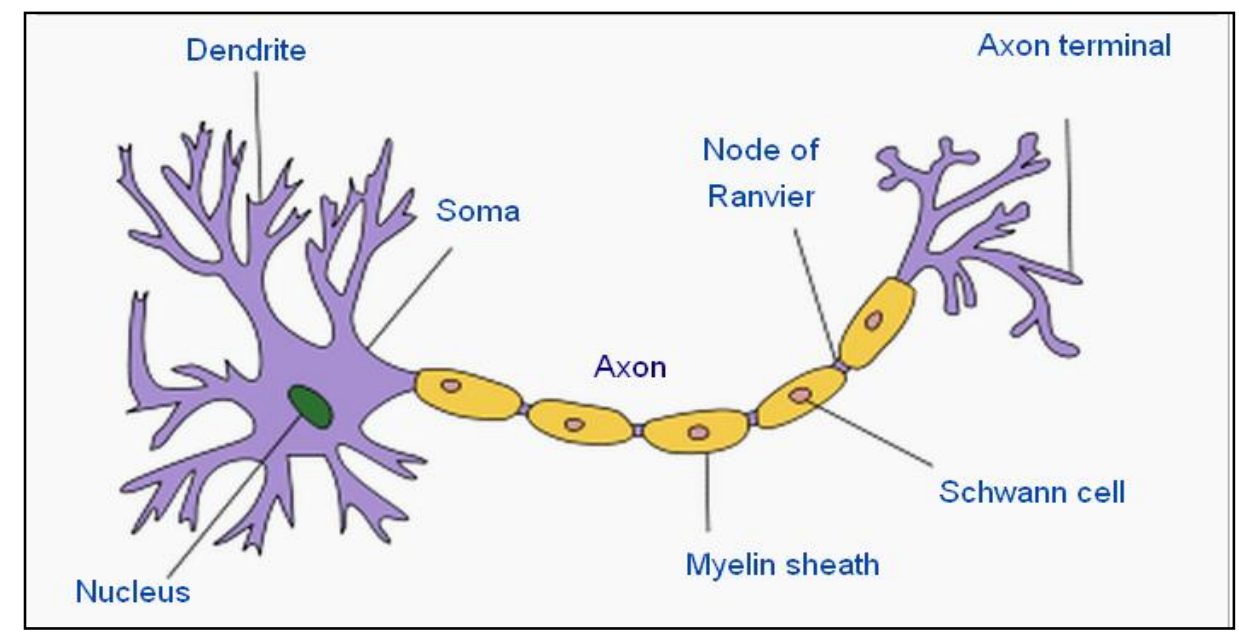

Figure 6 An example of a neural cell, neuron (Adopted from [14])

The main parts of neural cell are: cell body, dendrite, and axon (Figure 6). Dendrites and axons are special types of extensions found in neural cells whose main function is establishing connections and conducting neural impulses to the other neurons in the system. Connections established between the neurons are called synapses. Usually, axons of one neuron create synaptic connections with dendrites of another neuron. In their nature, signals transmitted by neurons represent trains of electro-chemical pulses [8]. The neuron that sends the signal is called presynaptic, while the neuron receiving the signal is called postsynaptic [14]. Based on the type and the strength of the synaptic connection postsynaptic neuron can act excitatory, inhibitory or modulatory on the transmitted signal. In biological systems, based on the signal transmitting activity lifelong changes on synaptic connections are induced. Scientists believe that this is where the learning and memorizing ability of the biological neural network systems lies.

The human brain is estimated to have between 10 and 500 billion neurons forming very complex neural networks where one neuron can be interconnected with hundreds to thousands of other units [8]. Thanks to this highly complex structure and parallel information processing we have the ability to speak, think, recognize, learn... Most importantly we are able to perform many highly complex actions in a routine manner. Actions that, if performed by the computers that 
have about million times faster processing ability, would require lots of complex and coordinated calculations that would take a lot of time to solve [8].

\subsubsection{Artificial Neural Networks}

Artificial neural networks (ANN) are computational concepts inspired by the biological neural networks, or biological intelligence. The main idea behind the development of ANN's is creating adaptive computer systems, which have similar learning ability as biological neural networks. Logical computer realizations (software) of ANN's are based on statistical and signal processing concepts rather than actual biological mechanisms [14].

When observed structurally ANN's just like biological neural systems, are constructed of logically interconnected processing units, artificial neurons. Schematic representation of artificial neuron is given in figure 2. Artificial neurons are capable of receiving and sending signals to other artificial neurons through logical connections (green lines on figure 7 ) that roughly can be compared to biological neural synapses. Each connection is determined by specific numerical value, called "connection weight" $\left(W_{i}\right)$, which multiplies the transmitted signal $\left(I_{i}\right)$ before it is passed to the receiving artificial neuron (equivalent to postsynaptic neuron in biological systems). Output of the artificial neuron is produced by applying predefined activation function on the summed value of input signals $\left(\Sigma I_{i} W_{i}\right)$. In this way produced, output is transmitted to the following logically connected neuron(s) in the sequence, within the ANN (pink line on figure 7). 


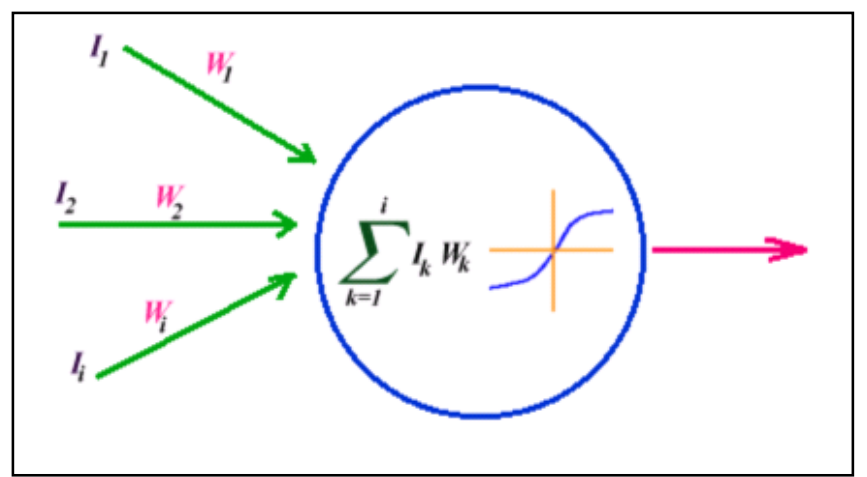

Figure 7 Schematic representation of artificial neuron (Adopted from [8])

Most commonly used activation functions for signal processing within the artificial neurons are: "Piecewise linear activation function" and "Sigmoid (Logistics) activation function".

Sigmoid activation function is determined by the following mathematical relation:

$$
f(x)=\frac{1}{1+\exp (-x)}
$$

This function is also known as the S-curve function, since Cartesian graph of this function takes shape in the form of letter "S". (Figure 8, right)

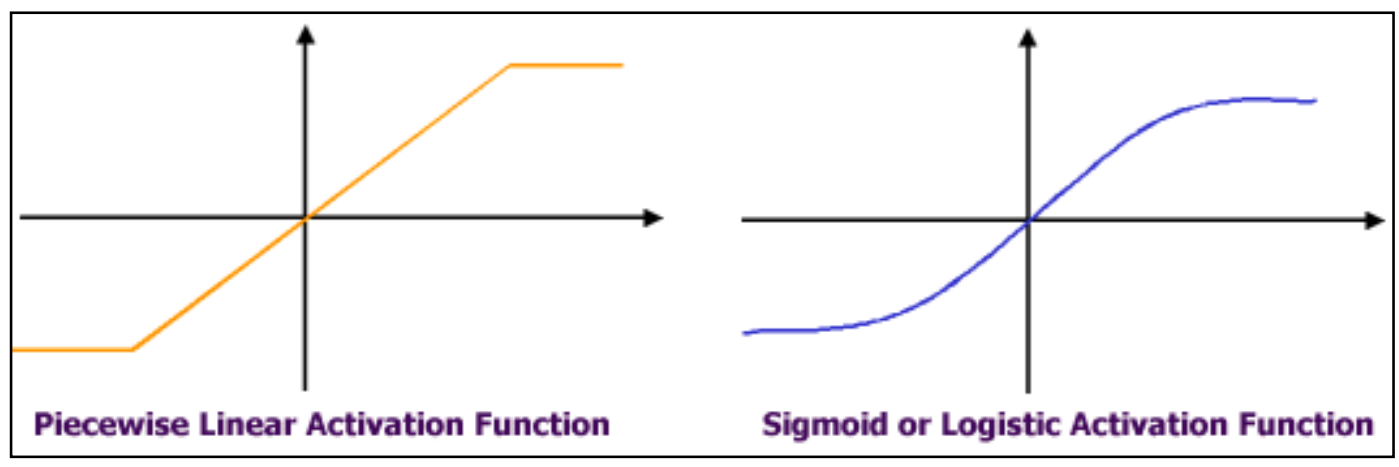

Figure 8 Most commonly used activation functions (Adopted from [8])

When it comes to ANN assembly, most typical topographies organize artificial neurons into three layers. First layer is the input layer in which number of neurons corresponds to the number of inputs provided to the ANN. When roughly compared to the biological neural systems this 
layer would correspond to an array of sensible neurons located within the senses (eye, ear...), that generate input signal or excitation for the central nervous system. Middle or hidden layer is where the most of the functional relationships between the input and output data are being extracted during the neural network training process [8]. This sequence of ANN assembly would roughly correspond to the biological neurons located within the central nervous system that processes received information/signal and produces appropriate reactions or the outputs. Finally, third layer of the most common ANN topography is the output layer, which much like the input layer contains specific number of neurons corresponding to the number of desired ANN outputs.

\section{(Figure 9)}

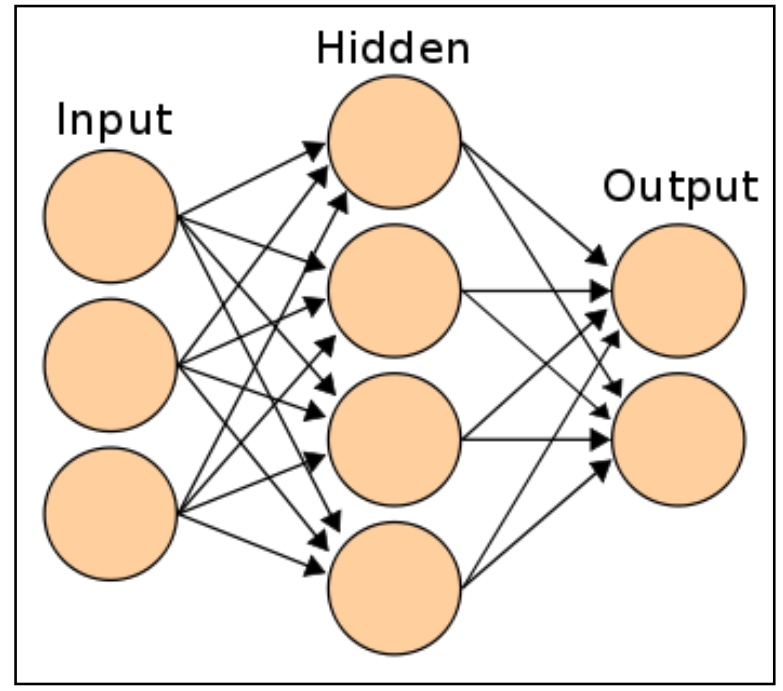

Figure 9 Layered structure of the Artificial Neural Networks (ANN) (Adopted from [14])

It is important to mention that ANN's can have multiple outputs, as shown in Figure 9. As said in section 1 of this chapter, it is believed that biological nervous systems learning ability lies in the activity based induction of permanent changes on the synaptic connections that influence the transmission of neural signals between the neurons. Similarly, ANN's learn by adjusting weights of logical interneuron connections through process called "neural network training". In general there are three types of neural network training: supervised, unsupervised, and reinforced. 


\section{Supervised training}

Supervised training paradigm is actually neural network training with feedback. This concept requires a set of known inputs and their corresponding outputs to be provided to the network during learning phase. When interneuron connection weights are clamped, error between network produced output and desired (provided) output (corresponding to particular input values) is estimated. Training continues by changing weights of networks interneuron connections in a way such that the error between provided and neural network produced outputs (ideally) converges to zero. This concept is often called training with a help of a teacher. Scheme of this procedure is shown in figure below.

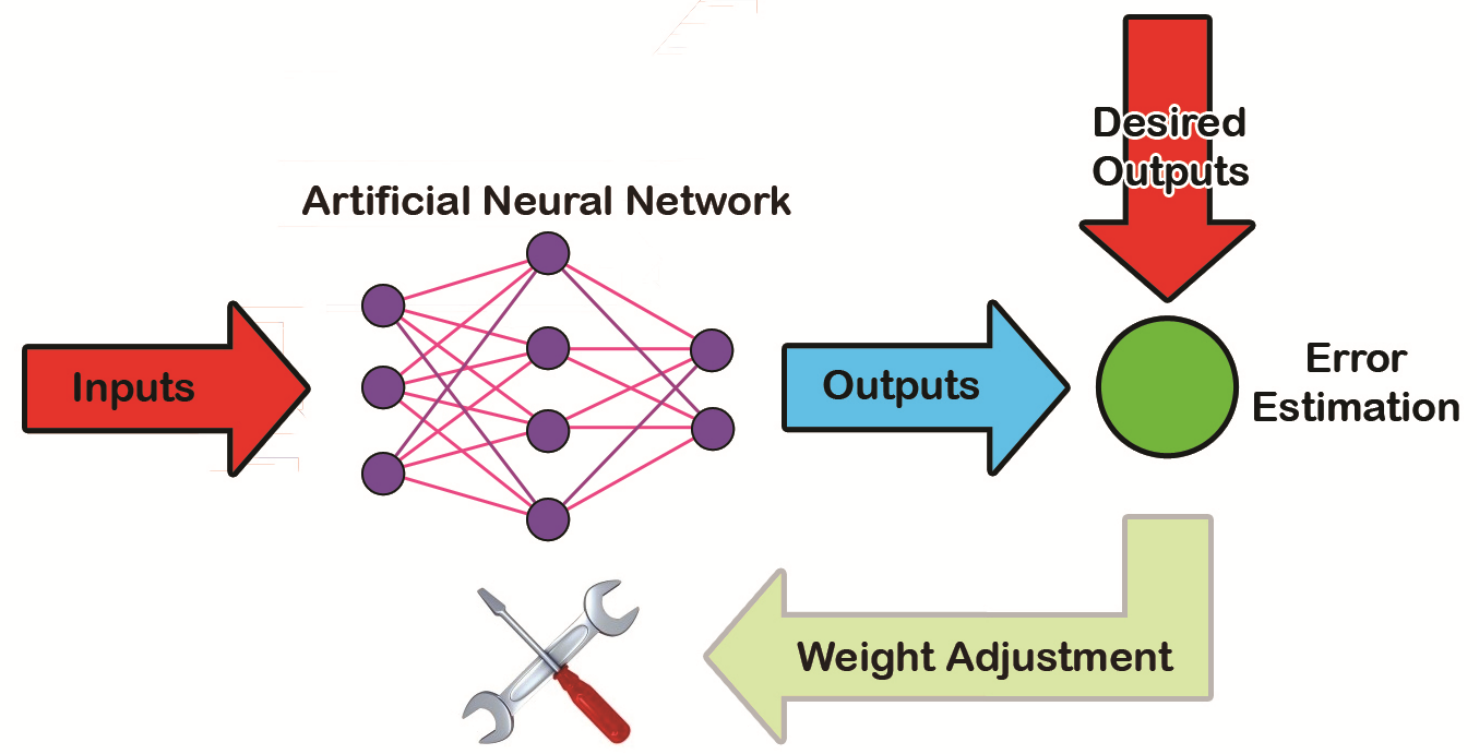

Figure 10 Supervised neural network training scheme

Supervised training paradigm is used in solving tasks like: speech recognition, pattern recognition (classification), and regression or function approximation. Most of the neural networks applications in petroleum engineering are using supervised learning paradigm [8]. The most widely used supervised learning algorithm is the Backpropagation algorithm, which will be explained in more details in section 2.2 .3 of this chapter. 


\section{Unsupervised training}

With this training procedure, neural network is provided only with input patterns. Therefore there is no feedback to the network, or in other words, when this concept is applied network does not benefit from the teacher. Networks trained with this method have the objective to find hidden patterns in unlabeled data by self adaptation. Unsupervised training is used for classification problems (clustering), pattern recognition in unstructured data, dimensionality reduction...

\section{Reinforced Training}

Is a special type of learning paradigm which can be classified as an intermediate form of Unsupervised and Supervised learning paradigms. Here just like in the case of unsupervised learning, data is usually not given, but rather gathered by systems interaction with the environment. In other words, system produces and sends certain outputs to the environment and receives feedback. Feedback is estimated as either rewarding (good) or punishable (bad), and accordingly network parameters are adjusted. This learning paradigm is highly used in control problems, computer games, and sequential decision tasks [14].

\subsubsection{Back-propagation Neural Network (BACKPROP)}

Backpropagation learning algorithm is supervised training algorithm applied to the feed forward neural networks. Typical example of a feed forward neural network is given in Figure 9. As it can be seen on Figure 9 each neuron within the hidden layer receives inputs from all neurons of the input layer, and sends its outputs to all neurons of the output layer. The most specific feature of this concept is that there are no connections between neurons of the same layer. Signal in these networks travels from input through hidden layer, towards the output, and therefore the name "feed forward neural network". 
In supervised learning method, when neural network training begins, connection weights are given some initial value. Output produced with such network is compared to the desired output, and error signal is calculated. Backpropagation algorithm propagates error signal backward through the network to each of the networks units and calculates appropriate weight changes, in order to reduce outputs error. New output is produced with the connection weight adjusted network, and the whole procedure of output error estimation and error Backpropagation is repeated. This whole procedure is repeated until the network learns the problem "well enough", or in other words when overall error between the network outputs and desired outputs does not fall below some predefined threshold. 


\section{METHODOLOGY OF THE TOP DOWN INTELLIGENT RESERVOIR MODELING}

Top-Down Intelligent Reservoir Modeling is a recently developed reservoir modeling technique that approaches reservoir simulation and modeling from a somewhat different angle than conventional numerical reservoir simulation. In recent years there were many publications about the applicability of Top Down Intelligent Reservoir Modeling to different reservoirs, as well as verification of the technology vs. conventional reservoir simulators $[7,17]$.

Conventional reservoir modeling based on numerical simulation, starts by developing geocellular models based on the geological interpretations of the modeled reservoir. In such static representation of the reservoir, fluid flow is modeled by numerically solving flow equations yielding to dynamic representation of the reservoir. Calibration of models developed in such way is performed through process called "History Matching" in which reservoir parameters are being changed until satisfying match with real production data is accomplished. Only at this stage it is plausible to use the developed model in field development strategies and for future performance forecasts. It is important to notice that functional relationships are not being modified in the history matching process, and that overall process requires a lot of time.

Top Down modeling approaches reservoir simulation and modeling, by starting with the actual field production data (production history), augmented with as much as possible information about the reservoir (well tests, seismic data, well logs...). Complex relationships between known reservoir parameters and recorded production history are deducted by combining state of the art 
in artificial intelligence and data mining with conventional reservoir engineering techniques ${ }^{3}$. This approach yields to full field representations of the reservoir, later used in development strategies and production forecasting.

Advantages of Top Down Intelligent Reservoir Modeling are:

- Very low data requirement ${ }^{4}$ (analysis is possible with only production data);

- Short time needed for the development;

- Simplicity of the analysis;

While disadvantages are:

- Not applicable to green fields ${ }^{5}$;

- Requires tens of wells (more than 35) and production history lasting for more than 2 years.

Top Down reservoir modeling methodology can best be summarized with the following flow chart (Figure 11).

${ }^{3}$ Decline and Type Curve analyses, and Single Well Numerical Simulations.

${ }^{4}$ In terms of data type, and variety of required data.

5 "Green fields" is a common name for new, completely undeveloped oil and gas prospects. Similarly, term "Brown fields" is used for the developed oil and gas prospects. 


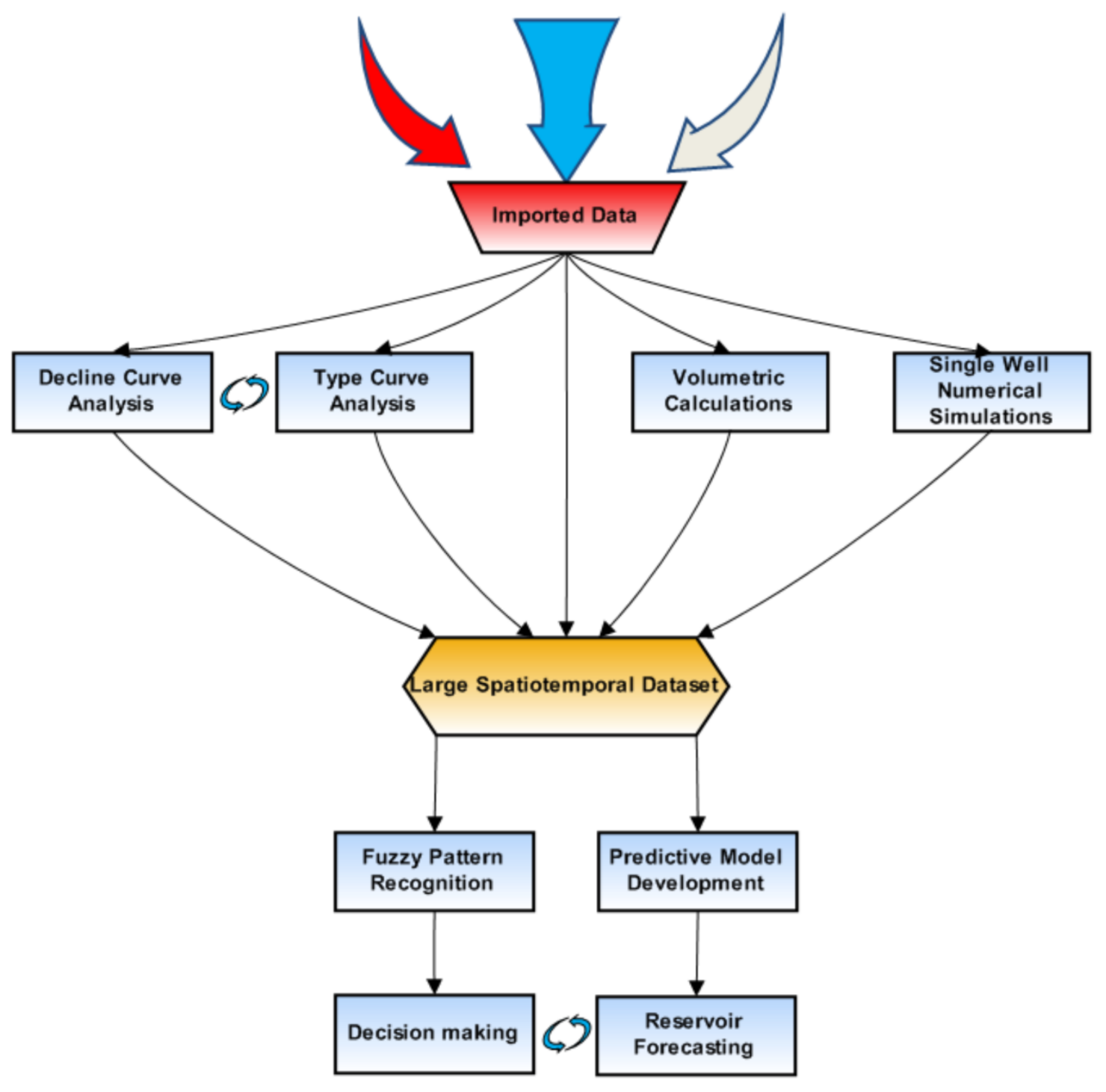

Figure 11 Flow Chart of Top Down Modeling

The main binder of all of the collected and generated data is neural network or in other words artificial intelligence. One thing that can be deducted from this is that there is no predetermined functional relationship that is creating a bound between reservoir properties and actual hydrocarbon production from the reservoir.

The following table explains in a best way, the differences between the Top Down Intelligent Reservoir Modeling and the numerical reservoir simulation. 
Table 2 Top Down Reservoir Modeling vs. Numerical Reservoir Simulation (Adopted from [12])

\begin{tabular}{|c|c|c|}
\hline & Numerical Models & Top Down Models \\
\hline Reservoir Characteristics & $\begin{array}{l}\text { Uncertiain: } \\
\text { - Measurements } \\
\text { - Interpretations } \\
\text { Subject to modification during } \\
\text { history matching }\end{array}$ & $\begin{array}{l}\text { Uncertain: } \\
\text { - Measurements } \\
\text { - Interpretations } \\
\text { Subject to modification during } \\
\text { history matching }\end{array}$ \\
\hline Functional Relationships & $\begin{array}{l}\text { Certain: } \\
\text { - Conservation of Mass } \\
\text { - Darcy's Law } \\
\text { Unchanged during history matching }\end{array}$ & $\begin{array}{l}\text { Uncertain: } \\
\qquad \begin{array}{l}\text { Relationship between } \\
\text { reservoir } \\
\text { characteristics and } \\
\text { production }\end{array} \\
\text { Subject to modification during } \\
\text { history matching }\end{array}$ \\
\hline
\end{tabular}

\subsection{Data Acquisition}

For this study gas production data was acquired from the EQT production company, a total of 77 well records were included in the database. For 76 out of 77 analyzed wells, well log records were found and downloaded from Kentucky Geological Survey's web site ${ }^{6}$. All of the downloaded well logs were in raster file format which made it impossible to automatically calculate different well log responses and perform mathematical manipulation in the available commercial software. Therefore, 65 out of 76 well $\operatorname{logs}$ were digitized with "NeuraLog" digitizing software and loaded into previously developed "Geographix Discovery" project of the study area. Since the majority of the analyzed wells were completed in the 1980's most commonly, well logs contained only GR, density, temperature, and caliper readings. In very few

\section{${ }^{6}$ http://kgs.uky.edu/kgsweb/DataSearching/OilGas/OGSearch.asp}


of the analyzed wells, neutron porosity, deep induction, resistivity, and gas detection logs were recorded. As stated in Chapter 2, sudden increase in GR readings accompanied by sudden decrease in density readings was considered to be a clear indicative of the Lower Huron Shale. For the operators drilling these wells in the 1980's this represented minimum information needed for the identification of completion target (Lower Huron shale).

Distribution maps of acquired and generated parameters at this stage were produced with geostatistical methods. Figure 12 shows gamma ray (GR) and density (RHOB) responses distribution maps throughout the study area. Figure 13 gives more insight into criteria used for picking Lower Huron shale interval in Geographix Discovery software. Finally, Figure 14 shows one cross section generated in East-West direction throughout the study area.

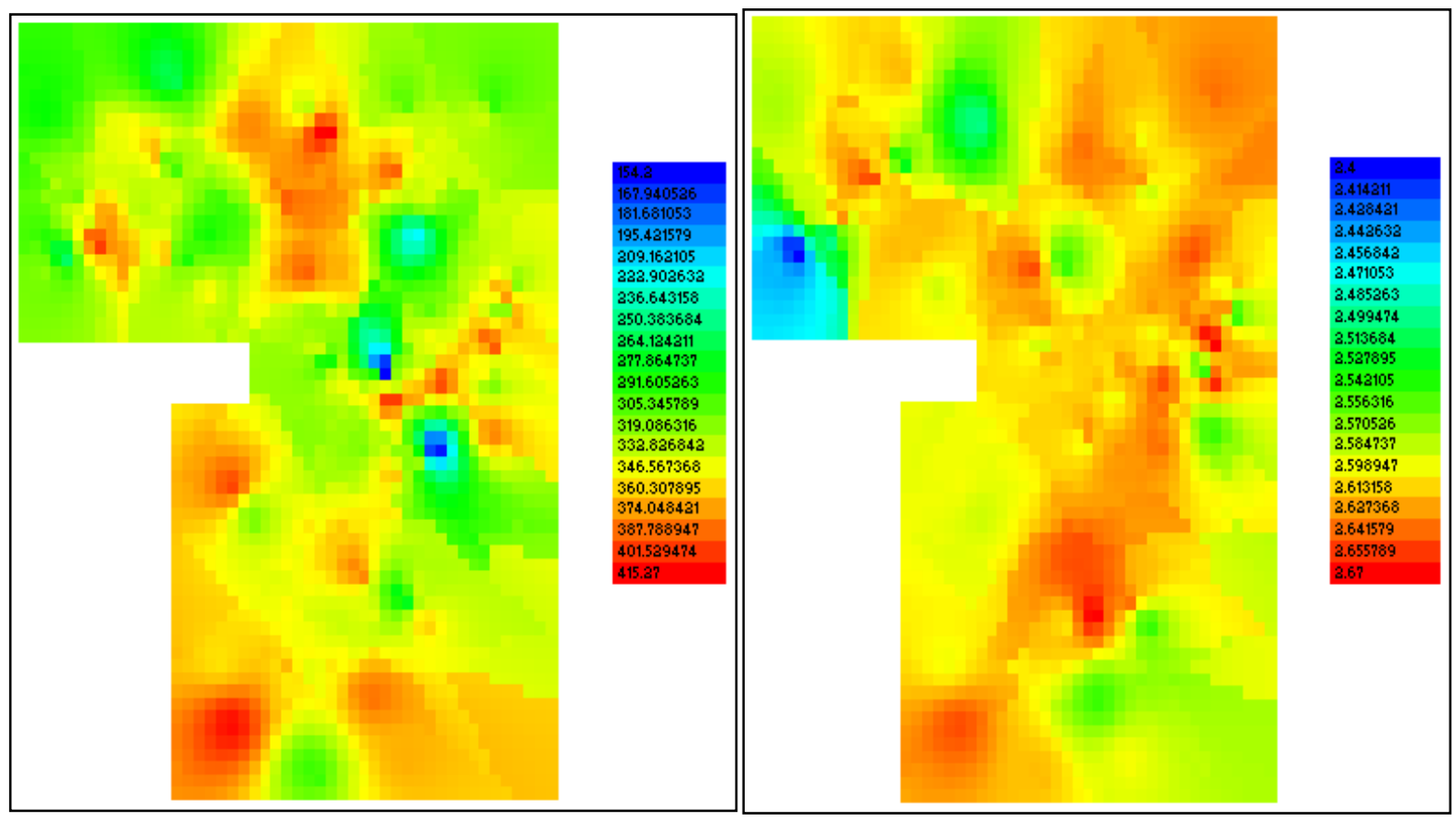

Figure 12 Gamma Ray (left) and Density (right), response distribution throughout the study area 


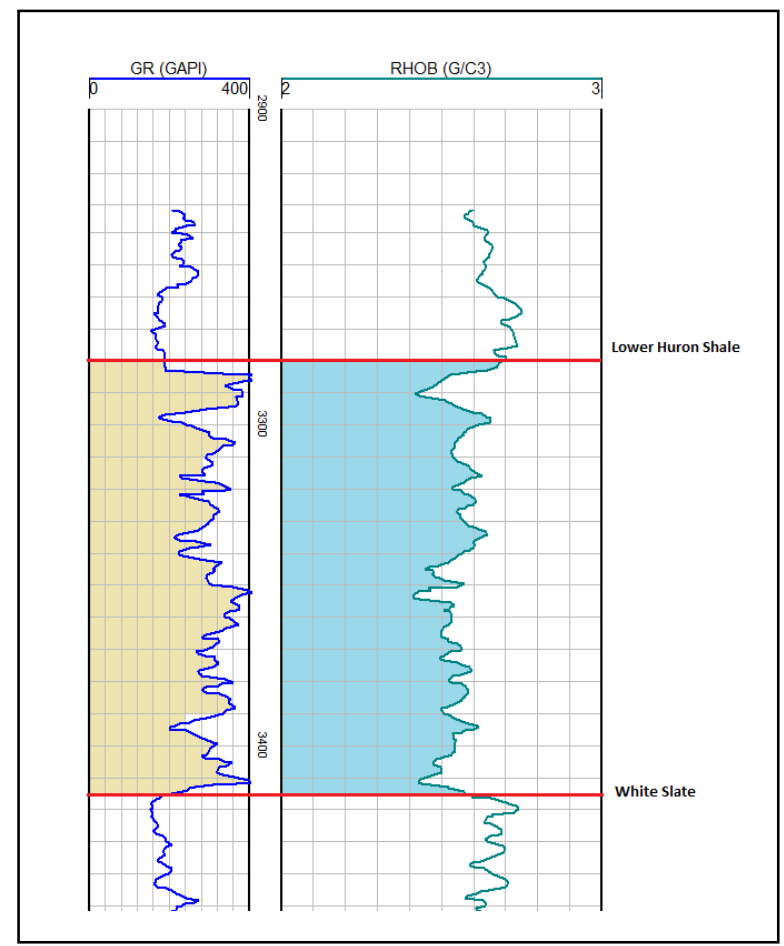

Figure 13 Typical well log response of the Lower Huron shale in the study area. (from10])

The next two steps of Top Down Intelligent Reservoir Modeling represent iterative process in which an attempt is made to accomplish same or close EUR with two different reservoir engineering techniques. The following next two sections is an algorithm/flow chart that gives more insight into decline-type curve matching procedure. 


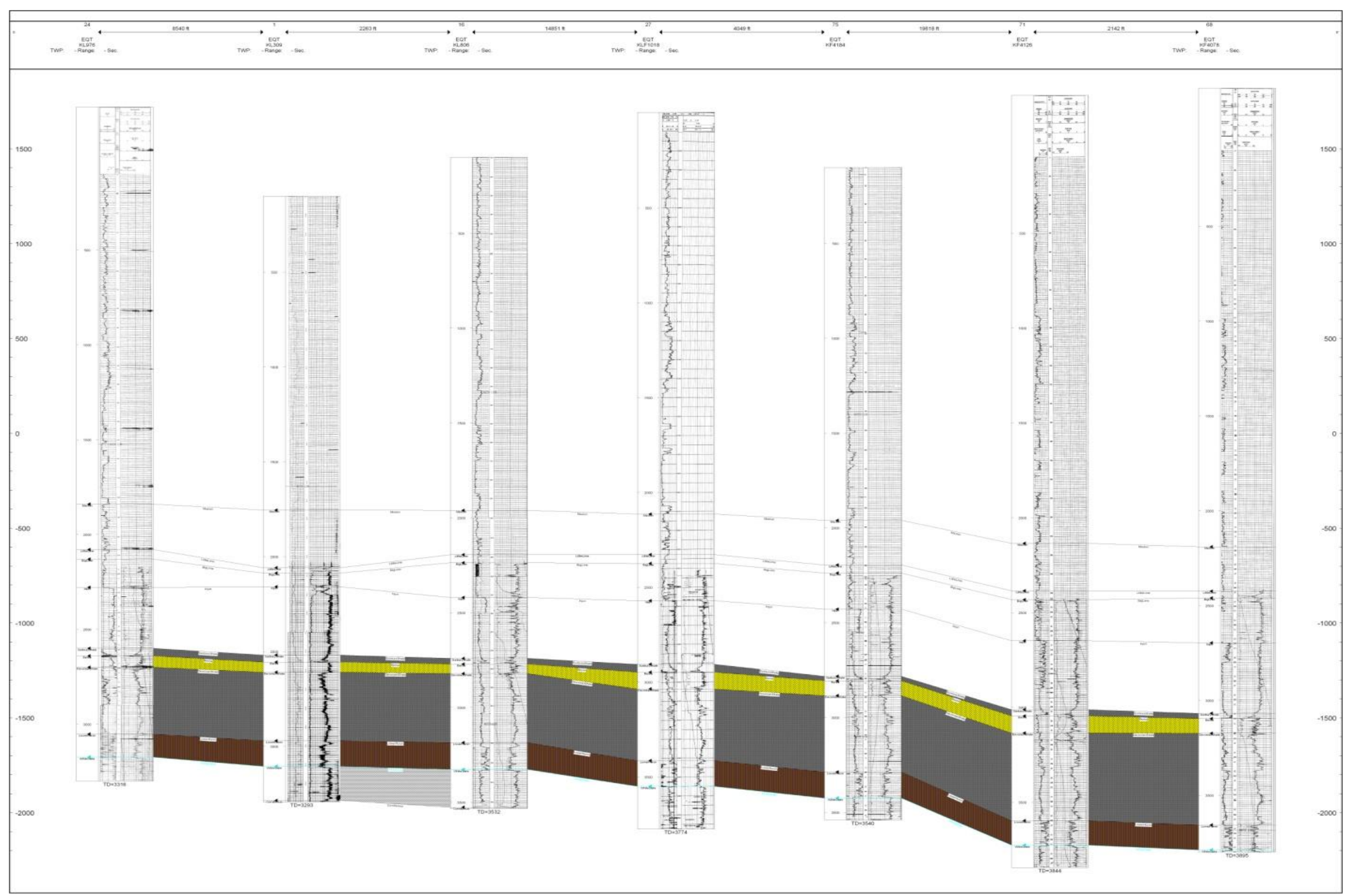

Figure 14 East West Cross Section of the Study area (Lower Huron Shale is painted Brown) 


\subsection{Decline Curve Analysis}

One of the simplest and most widely used reservoir engineering techniques for hydrocarbon production rate forecasting and estimation of hydrocarbon ultimate recovery is Decline Curve analysis. Basis of this method lies in curve fitting of a single line through the declining values of production rate (production history), and the assumption that the future trend of the fitted line would be the same or similar to the future trend of the declining production rate. Exactly this assumption enables quick prediction of the future production rate and estimation of the hydrocarbon ultimate recovery $(E U R)$ by simple extrapolation of the fitted curve to the point of abandonment rate ${ }^{7}$.

One of the biggest advantages of this method is that it is cost effective, easy to use, and very quick [1]. However, there are many disadvantages in using this unsophisticated approach. Some of which are: in most cases reservoir parameters cannot be quantitatively inferred based on the shape of the fitted decline curve, also changes in operating conditions (constrains) can significantly alter the shape of the decline, and therefore cause predictions made by the fitted curve to be highly inaccurate [1].

Conventional decline curve analysis uses mathematical relationship developed by Arps in 1940’s.

$$
q(t)=\frac{q_{i}}{\left(1+b D_{i} t\right)^{1 / b}}
$$

Where:

\footnotetext{
${ }^{7}$ Abandonment rate or economical limit is the point in well production history where the net income derived from the produced hydrocarbons equals to the expenses needed for well maintenance.
} 
$\mathrm{q}_{\mathrm{i}}$ - represents initial value of the decline curve;

$\mathrm{D}_{\mathrm{i}}$ - represents initial decline rate (unit of time) ${ }^{-1}$; and

$\mathrm{b}-$ represents decline exponent.

Based on the values of the b-decline exponent, there are three different forms of equation 1 , each characterizing one type of decline curve. Differences between the three types of decline curves are clearly observed on both Cartesian and semi-log graphs. Figures 15 and 16

\section{b=0 case - Exponential Decline}

For $b=0$ equation 1 reduces to the following form.

$$
q(t)=\frac{q_{i}}{e^{D_{i} t}}=q_{i} e^{-D_{i} t}
$$

This form of the equation 1 is known as the Exponential Decline. The previous equation represents equation of a straight line on semi-log graph (Figure 16).

\section{$0<b<1$ case -Hyperbolic Decline}

For values of $\mathrm{b}$ decline exponent falling between 0 and 1 Arps identified Hyperbolic type of the Decline Curve. The equation that determines this type of decline curve is exactly equation 1.

$$
q(t)=\frac{q_{i}}{\left(1+b D_{i} t\right)^{1 / b}}
$$

Shape of the Hyperbolic Decline curve in Cartesian and Semi-Log plots is given in figures 15 and 16 . 


\section{$b=1$ case - Harmonic Decline}

$b=1$ case is a special case of the Hyperbolic Decline, equation of this type of decline curve is given below.

$$
q(t)=\frac{q_{i}}{\left(1+D_{i} t\right)}
$$

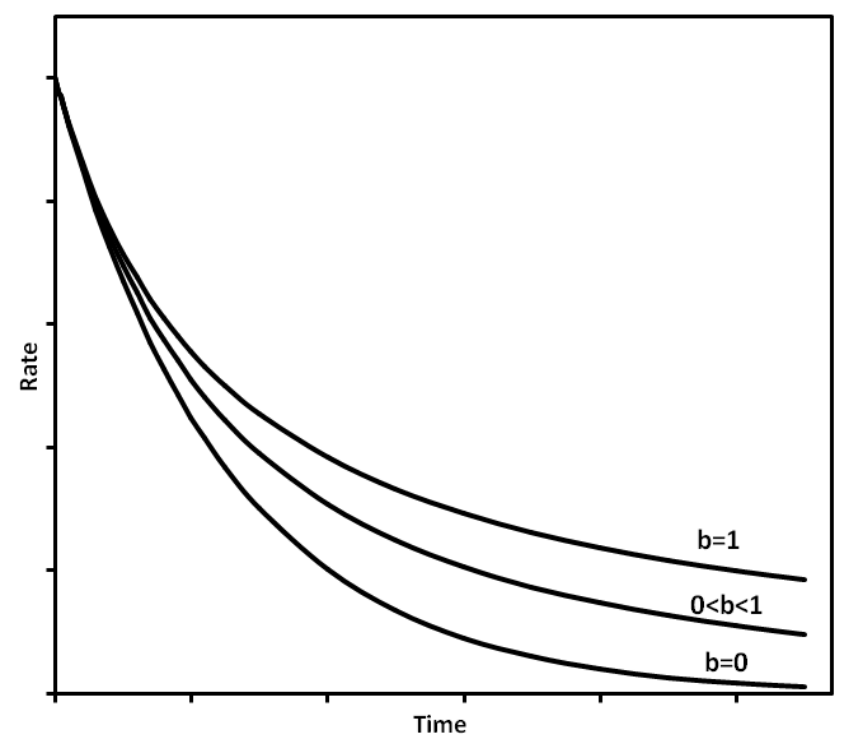

Figure 15 Cartesian plot of all three cases (types) of Arp's decline curve equation

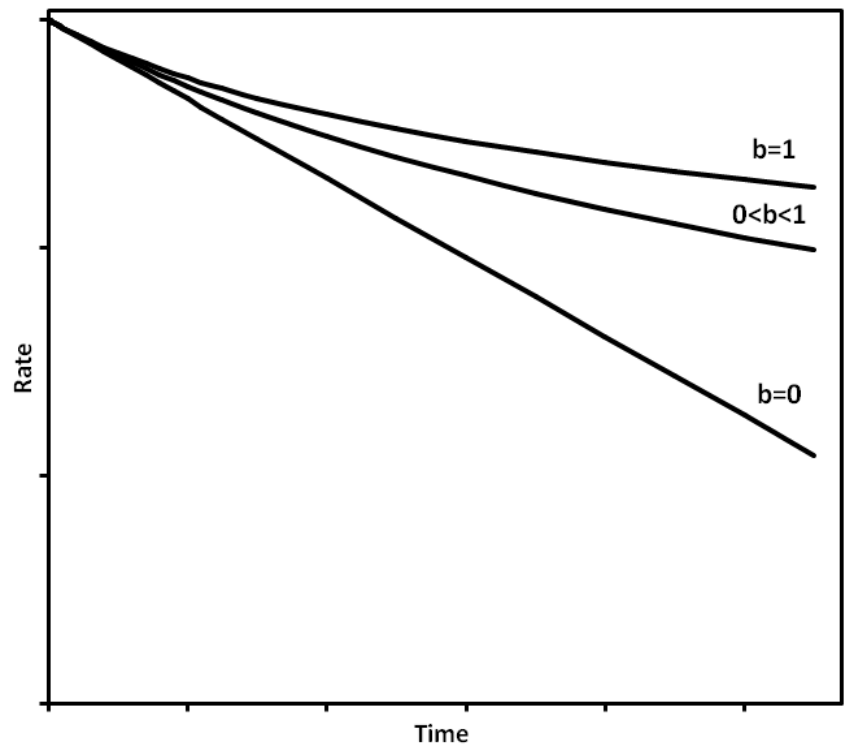

Figure 16 Semi-Log plot of all three cases (types) of Arp's decline curve equation 
Fitting a decline curve based on Arp's equation, over declining production rate values is performed by changing Qi, Di and b decline parameters until satisfying match with the historic production data is accomplished. IMAGINE ${ }^{8}$ software that was used in this study is capable of automatically identifying the most appropriate type of decline curve and selecting the most optimum combination of the three decline parameters. Other, very useful capability of this software is automatic calculation of estimated ultimate recovery $(E U R)$ based on the fitted decline curve, for any given abandonment rate.

Top Down Intelligent Reservoir Modeling methodology workflow requires decline curve matching to be performed before the type curve matching. This sequence is necessary due to input requirements of the available sets of type curves (Cox 1996) where b-decline exponent determines the most appropriate set of type curves to be used. More information about the utilized type curves, matching procedure and decline-type curve workflow is given in section 3.3. followed by a flowchart of decline-type curve matching procedure.

\subsection{Type Curve Matching}

Type Curve matching is an elegant tool for quick estimation of reservoir parameters, wellbore damage, and efficiency of different completion methods... Type curves were usually developed by analytically solving diffusivity equation for specific types of reservoirs or reservoir cases, computing plots for certain number of different scenarios and plotting the results in dimensionless domain on log-log plots. Theoretically shape of the actual well production history should match the shape of one of the curves in the type curve set developed for that specific type of reservoir. Matching is performed by plotting the original well production data on a log-log

\footnotetext{
${ }^{8}$ Software developed by the Intelligent Solutions Inc. (www.intelligentsolutionsinc.com)
} 
plot of the same cycle as the type curve plot, and by moving plot of actual data over the set of type curves until acceptable match is achieved. At the match points dimensionless values of the underlying type curve are recorded and reservoir parameters are back calculated by using formulas for the dimensionless values provided with the used set of type curves. The type curves used in this study were developed by Cox [3] in 1996 for hydraulically fractured wells completed in tight gas reservoirs.

One of the inputs for Cox's type curves is the b-decline exponent. Therefore, decline curve analysis is required as necessary method prior to using Cox's type curves. Matching with Cox's type curves in IMAGINE software is quite easy. Real production data (Green dots on Figure 17) are moved over underlying type curves until acceptable match is accomplished. While performing type curve matching an attempt is made to reach same or similar EUR as with the decline curve analysis for the same well. This way one method represents control environment for another which makes subjectivity induced errors less likely to occur.

If the same EUR cannot be reached with the type curve matching that means that decline curve analysis should be updated and type curve matching repeated for the new value of b-decline exponent. This procedure is repeated as many times necessary until acceptable matches are achieved.

Example of type curve matching procedure is shown in figure below. 


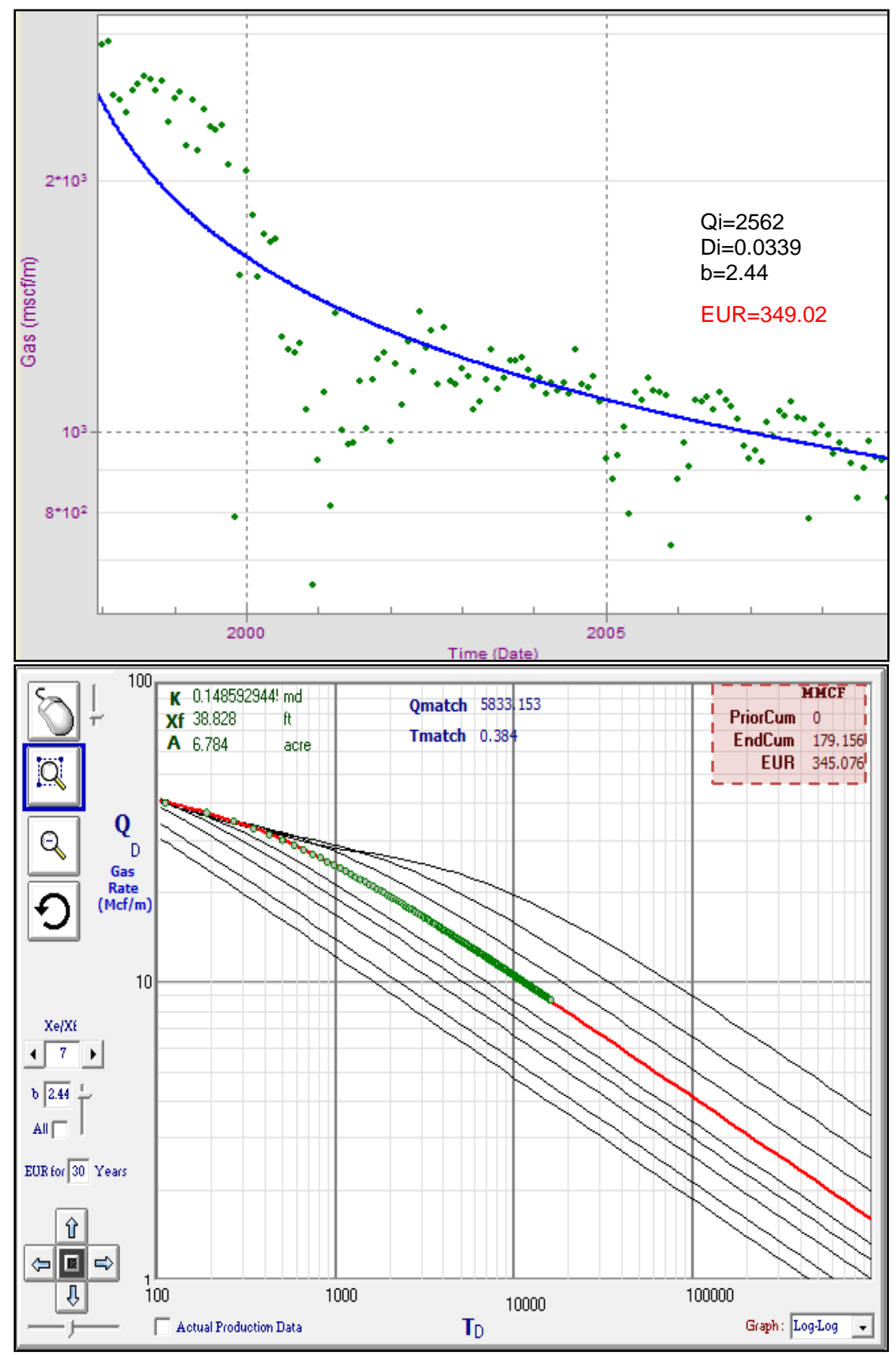

Figure 17 Example of type curve matching procedure (adopted from IMAGINE software) 


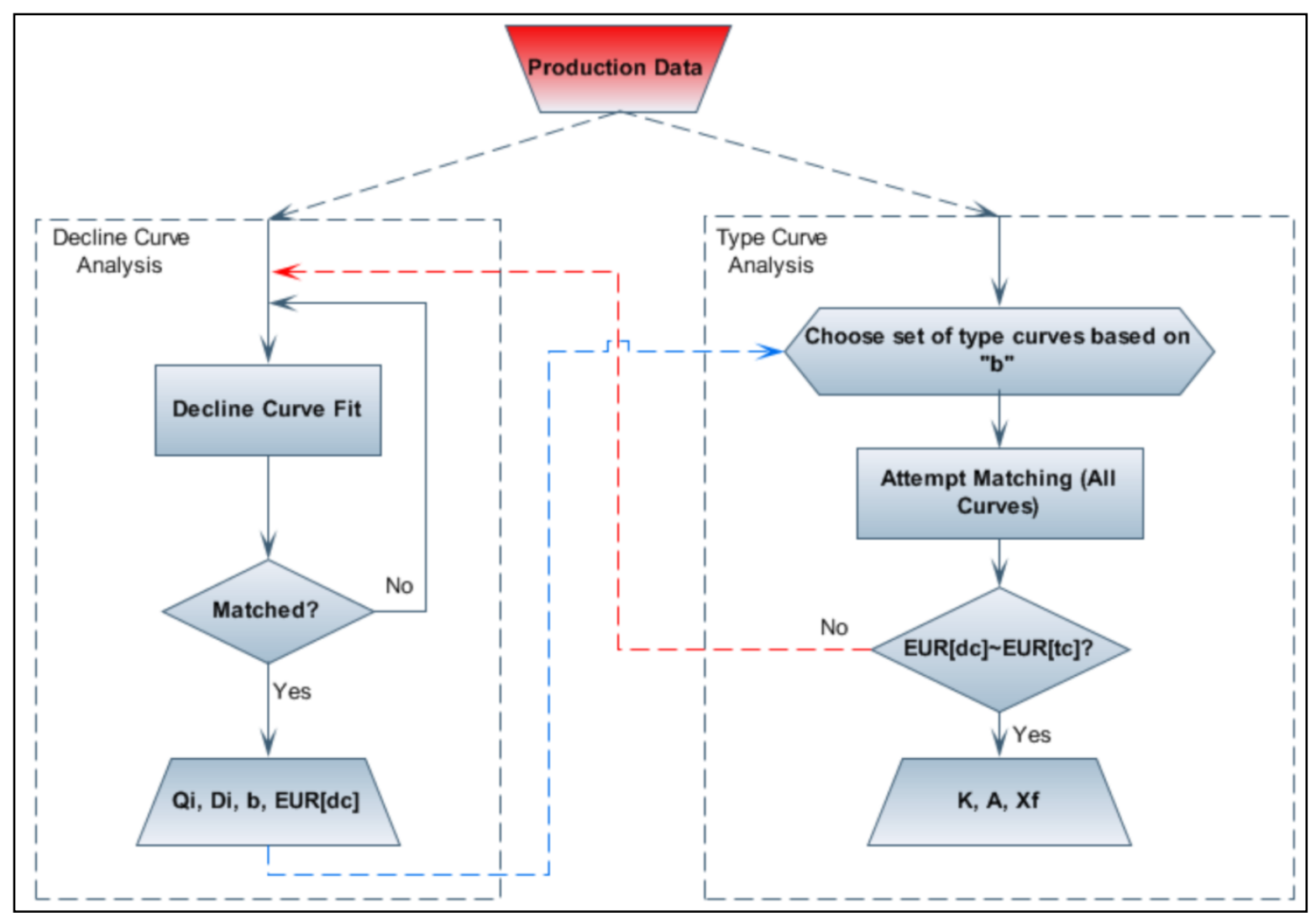

Figure 18 Flow Chart of Decline - Type curve analysis for a single well.

\subsection{Single Well Numerical Simulation}

Single well history matching as a part of the Top Down Intelligent Reservoir Modeling is performed in order to acquire more information about the studied reservoir. In this study single well history matching was performed with the Fracgen/Nfflow fractured tight gas and shale gas reservoir simulator package. This simulator was the most appropriate given the type of the analyzed reservoir.

History matching with Fragen/Nfflow starts with gathering as much as possible information about the fracture occurrences within the modeled reservoir. This information can be form the 
core analyses, FMI logs or outcrops of the formation ${ }^{9}$. Acquired fracture network data is provided to the Fracgen module of the Fracgen/Nfflow simulator package, which uses statistical methods to calculate the most probable representation of the modeled reservoirs natural fracture network. Fracture networks generated in this way contain information about the number of intersections between the fractures, apertures of every fracture within the network and spatial extension of the reservoir. Primary approach in Fracgen network generation required preparation of input text files for providing the software with the available information about the fracture network. This was followed by utilization of second module that was able to view the network. Whole method of preparing textual files, and using two different modules to observe the results made the whole process quite cumbersome. As a part of this study a small computer application (WVU-FGI) capable of preparing Fracgen textual input file, sending it to Fracgen and visualizing the results, was developed. With this application tedious textual input file preparations have been replaced with more user friendly Windows based interface. An example of fracture network generated in this study with Fracgen software is shown in figure below (Figure 19).

\footnotetext{
${ }^{9}$ Fracture occurrences observed in the outcrops are usually combination of the induced fractures by atmospheric alterations, and natural occurring fractures that are present throughout the reservoir.
} 


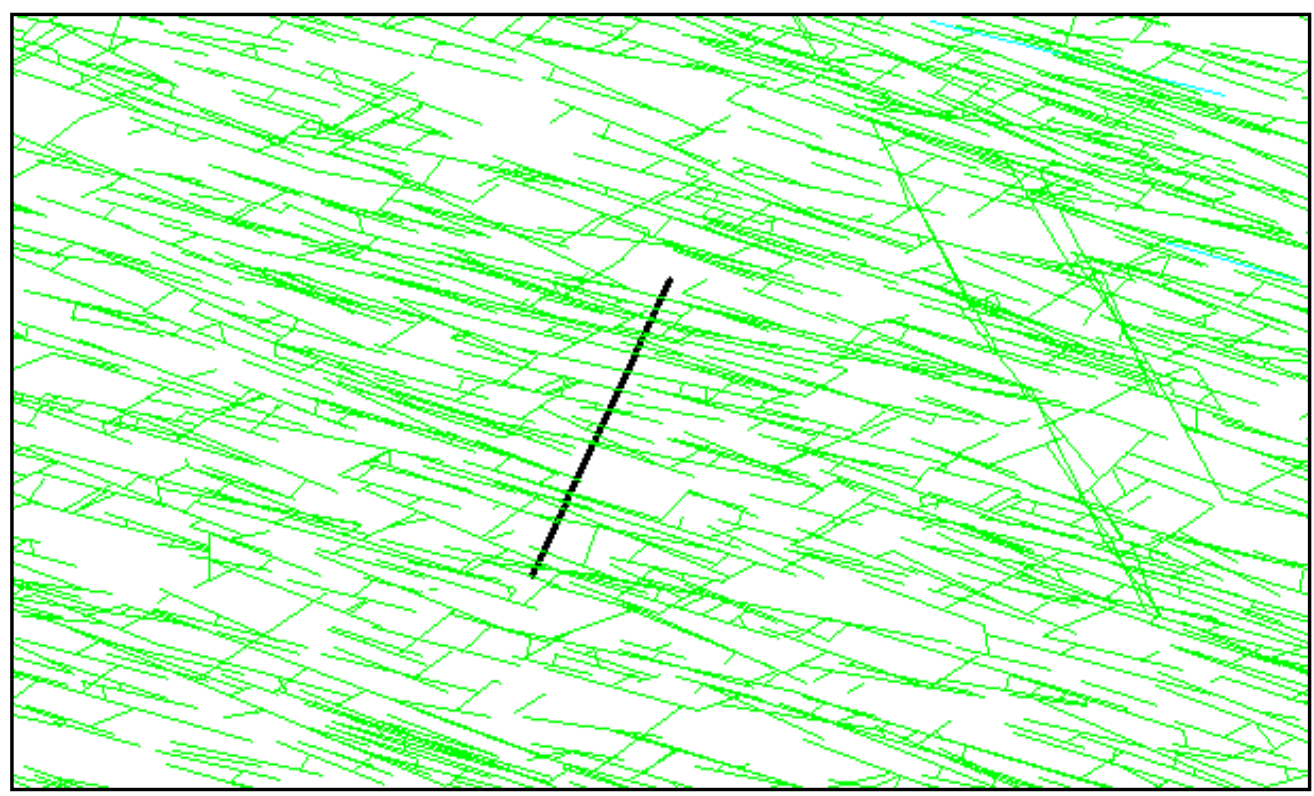

Figure 19 Representation of the most probable natural fracture network within the Lower Huron Shale in eastern Kentucky (generated with Fracgen).

The next step in Fracgen/Nfflow modeling is simulation of gas flow within the reservoir matrix and generated fracture network. Previously generated fracture network is loaded into the Nfflow module along with the fluid and reservoir properties (matrix), well location, simulation dates, and operational constrains $(B H P$ or $Q)$. With the information provided, Nfflow software simulates gas production from the reservoir. In this way generated production profile of each well is plotted against the real production data, if the match is within satisfying range of error, history matching for the specific well is considered to be completed. Otherwise, reservoir parameters like matrix permeability, matrix porosity, gas content, or natural fracture apertures are changed and the whole simulation process is repeated.

Figure 20 shows an example of a history matched well with the Fracgen/Nfflow software package. 


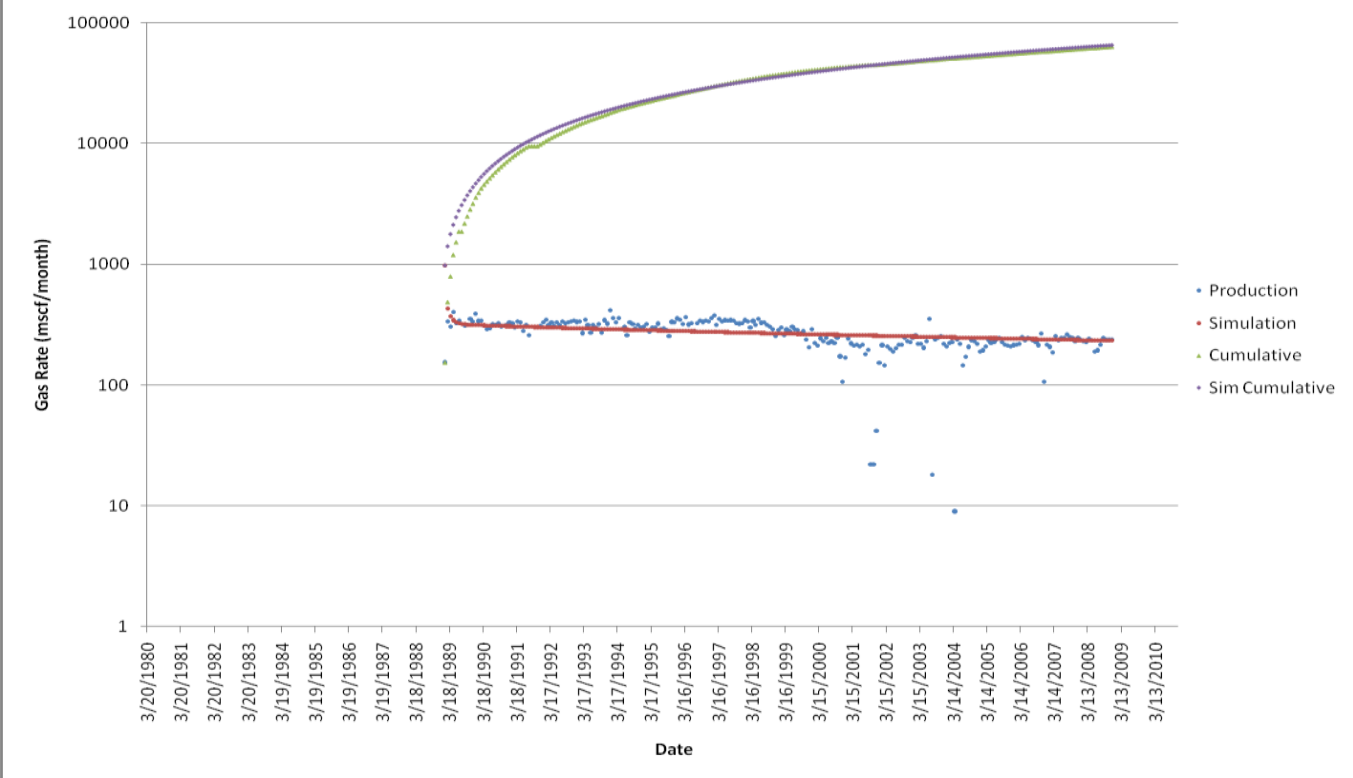

Figure 20 Example of a history matched production data with the Fracgen/Nfflow simulator.

\subsection{Geostatistics}

Probability distribution maps of each of the imported and derived parameters were produced with geostatistical methods (Nearest neighbor, Krigging...). There are two objectives in producing such maps. The first objective is to give more insight into spatial distribution of each of the observed geological parameters (Porosity, Permeability, Thickness, depth...), and since the final objective of the Top Down Intelligent Reservoir Modeling is the development of a full field predictive models by using neural networks, the second purpose of geostatistics is database completion or database patching ${ }^{10}$.

Snapshots of geostatistically produced maps are given in figures below.

${ }^{10}$ Training of the neural networks with the incomplete datasets is impossible. Incomplete datasets are usually patched with AI based methods, or simply by performing geostatistics (interpolation). 


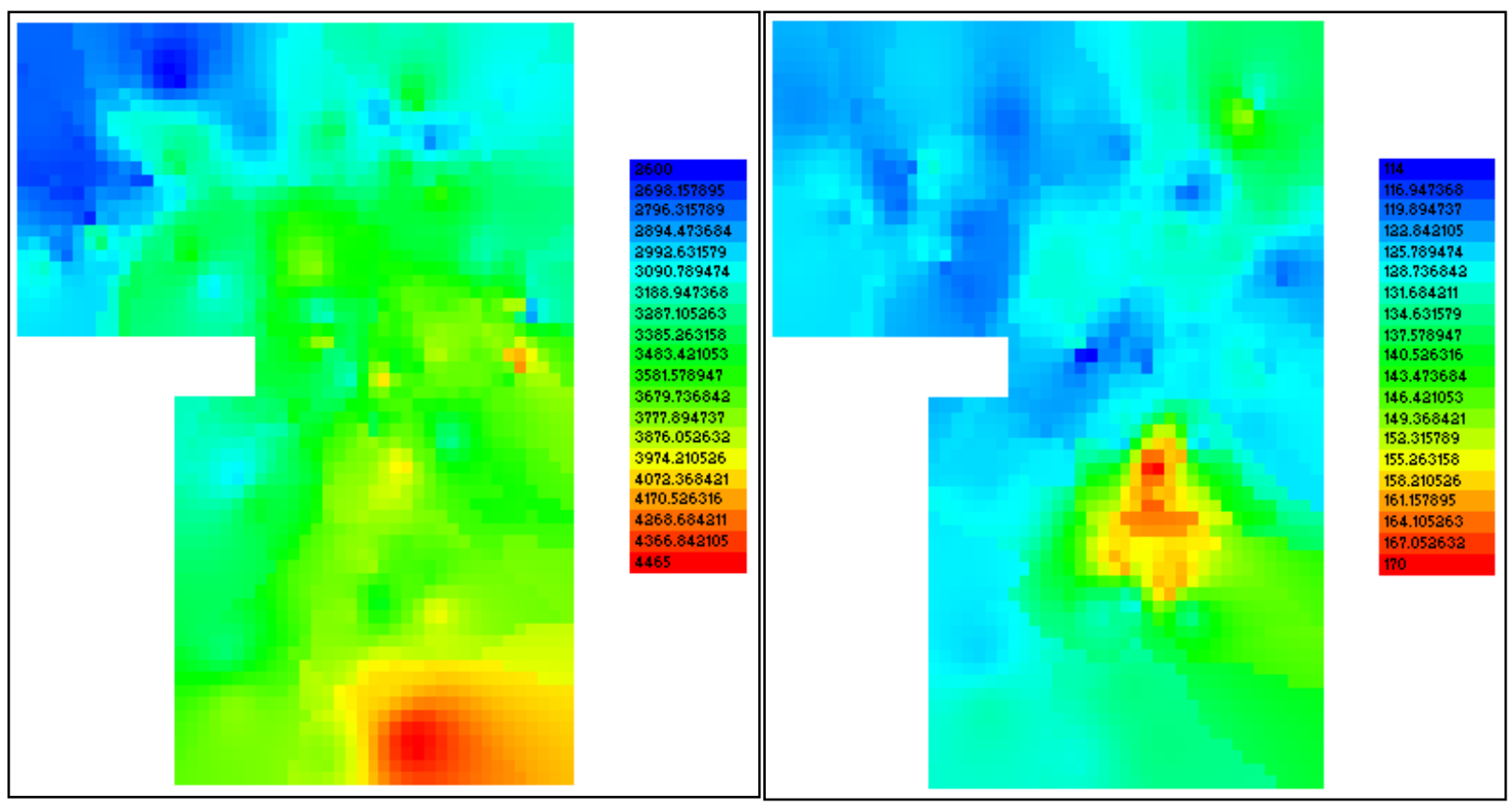

Figure 21 Geostatistically produced map of formation depth (left) and formation thickness (right)

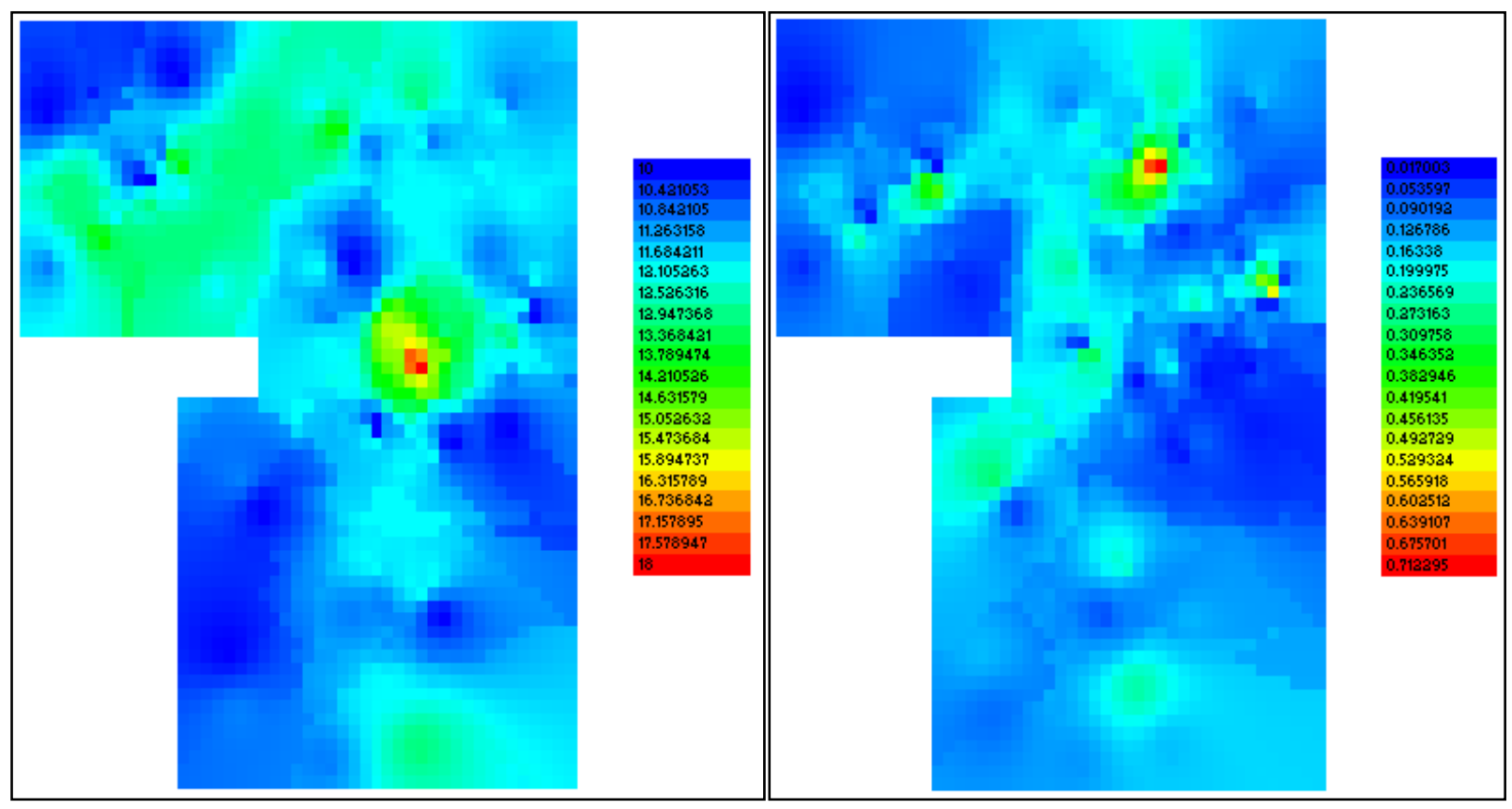

Figure 22 Spatial distribution maps of Log Porosity (left) and Formation permeability from type curves (right) 


\subsection{Fuzzy Pattern Recognition (FPR)}

Fuzzy pattern recognition is the first implementation of artificial intelligence in the Top Down Intelligent Reservoir Modeling workflow. Fuzzy Pattern recognition $(F P R)$ is technology capable of deducting patterns from very complex data. In Top-Down Intelligent Reservoir Modeling methodology, FPR is used for deducting complex spatial distribution patterns of reservoir productivity indices (First 3,6,9 months of production, remaining reserves, cumulative production...).

Procedure of Fuzzy Pattern Recognition is as follows. Analyzed productivity index is plotted in two directions, $\mathrm{X}$ (Longitude), and Y (Latitude) directions. 1D distribution plots produced in this way usually have patterns that seem nondeductible to the human eye. That is why fuzzy pattern recognition technology is very useful. As said before FPR is capable of deducting complex spatial distribution patterns of the observed parameter. On Figure 23 in the part named "Delineated Fuzzy Pattern in Longitude Direction" an example of this procedure is shown. Plot shows actual data represented by gray dots and deducted fuzzy pattern (purple dots). Next step of this procedure would be delineation of the FPR curves in both Latitude and Longitude directions. Reservoir delineation is performed by moving two lines over Lat. and Long. FPR curves, (Upper and Lower Delineation Lines, Figure 23), fitting straight lines through their intersections and extending them over 2D map of a reservoir (Figure 23 - "2D Reservoir Partitioning”). In this way reservoir is partitioned into 5 zones of different relative reservoir quality $(R R Q)$.

The dark brown squares in $2 \mathrm{D}$ delineated reservoir plot represent zone of highest relative reservoir quality index (RRQI 1). In Top Down Intelligent Reservoir Modeling terminology this zone is named "High-High" because it is determined by the lines passing through the 
intersections of the upper delineation lines with the FPR curves. Similarly, other four reservoir quality zones are identified as "High-Mid", "High-Low \& Mid-Mid", "Mid-Low", "Low-Low" (Figure 23).

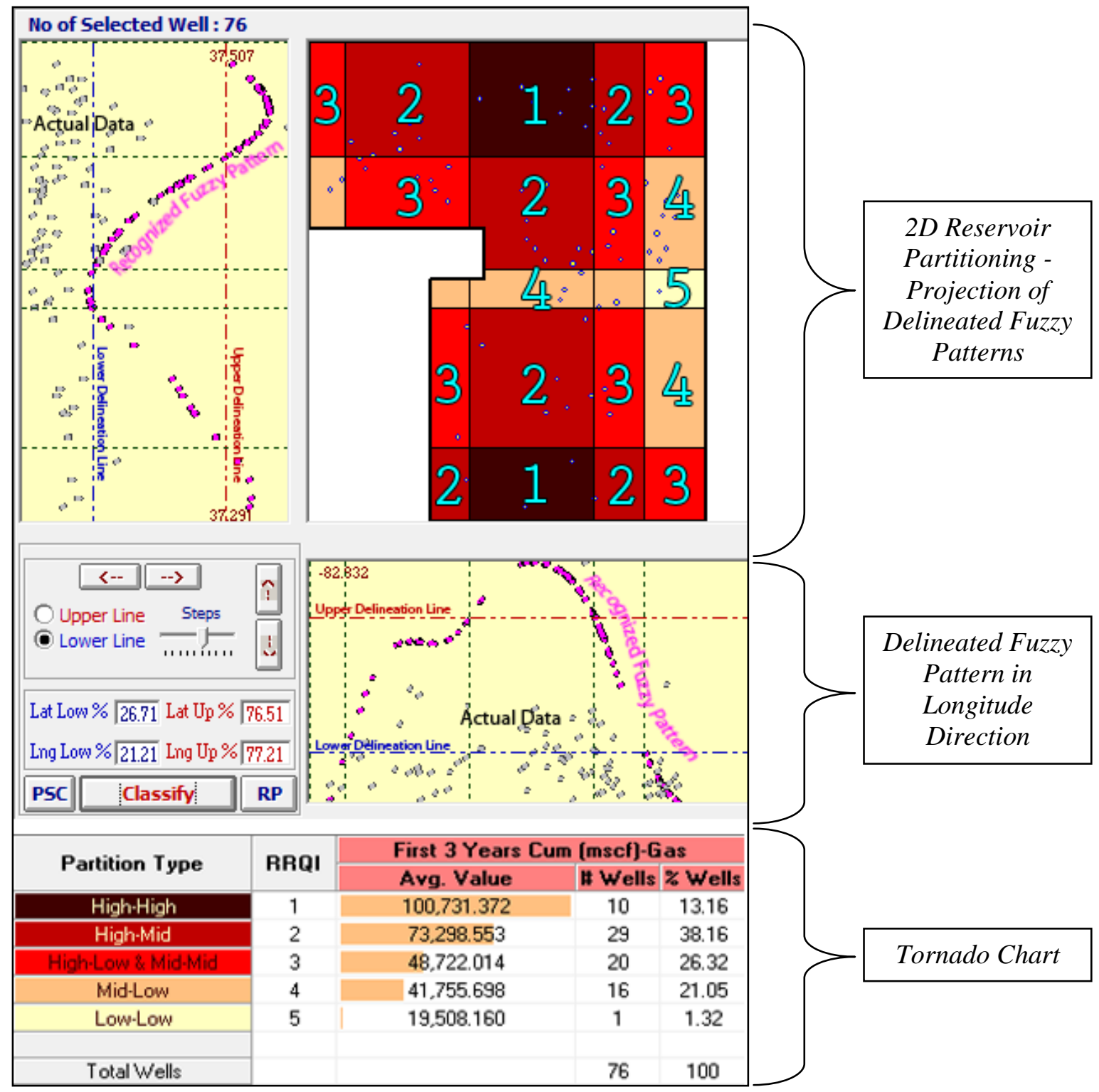

Figure 23 Detailed explanation of Fuzzy Pattern Recognition Methodology

Delineation is performed until the shape of what's called "Tornado Chart" on Figure 23 does take tornado like structure, or in other words until average values of the observed parameter start to monotonically decrease from the zone of highest to the zone of lowest quality (Figure 23). 
Fuzzy Pattern Recognition model is calibrated with the most recently drilled wells by removing them from the analysis, once again delineating the reservoir and attempting performance prediction for the removed wells.

This methodology enables identification of the "sweet spots" within the reservoir, and therefore it represents a very useful tool for field development decision making.

Before decision making takes place, FPR plots need to be calibrated with the most recently drilled wells. This is performed by removing such wells from the analysis (not more than 10\% of them), once again identifying fuzzy patterns and performing delineation in a way such that removed wells fall into appropriate zone of reservoir delineation based on their specific values of the observed parameter (for example first year cumulative production)

\subsection{Predictive model development}

Predictive models are intelligent systems based on artificial neural networks that are capable of accurately predicting hydrocarbon production rate from any of the existing or future drilled wells within the modeled reservoir.

Heart of the predictive models, are efficiently trained neural networks. Neural networks used in predictive modeling are trained with Backpropagation algorithm or in other words with supervised learning methodology. Efficient training of the neural networks highly depends on the quality and completeness of the datasets used for their development. The objective of the

previously described steps of the Top Down Intelligent Reservoir Modeling methodology is acquisition/derivation of as much as possible information about the modeled reservoir. This information is further passed to the neural networks, which deducts complex relationships between the production rate and provided reservoir parameters, during neural network training 
procedure. Successful completion of this process highly depends on the quality of the information/data used for neural network training. The following sections of this chapter give more details about the whole neural network development procedure.

\subsubsection{Database preparation}

In database developed for predictive model development each entry contained information estimated at one specific well and its closest two offset wells at specific time step. Well based information contained in each entry is as follows: latitude, longitude, porosity, permeability, production rate at previous time step, production rates from previous two time steps, cumulative production at previous time step, and date of first production. The same entry contains the same type of information from two offset wells. The time span of the database developed in this study is from January 1985 to December 2008 (last data entry).

\subsubsection{Key Performance Indicators Analysis (KPI Analysis) ${ }^{11}$}

Key performance indicator analysis is a very useful tool developed by the Intelligent Solutions Inc. that is able to identify degree of influence of each of the imported input parameters on the desired outputs of the to be trained neural network. In the case of Top Down Intelligent Reservoir Modeling, Key performance Indicators analysis successfully identifies the most influential parameters (from the provided database) on the production rate that is being predicted.

\footnotetext{
${ }^{11}$ Technology developed by the Intelligent Solutions Inc.(www.intelligentsolutionsinc.com)
} 


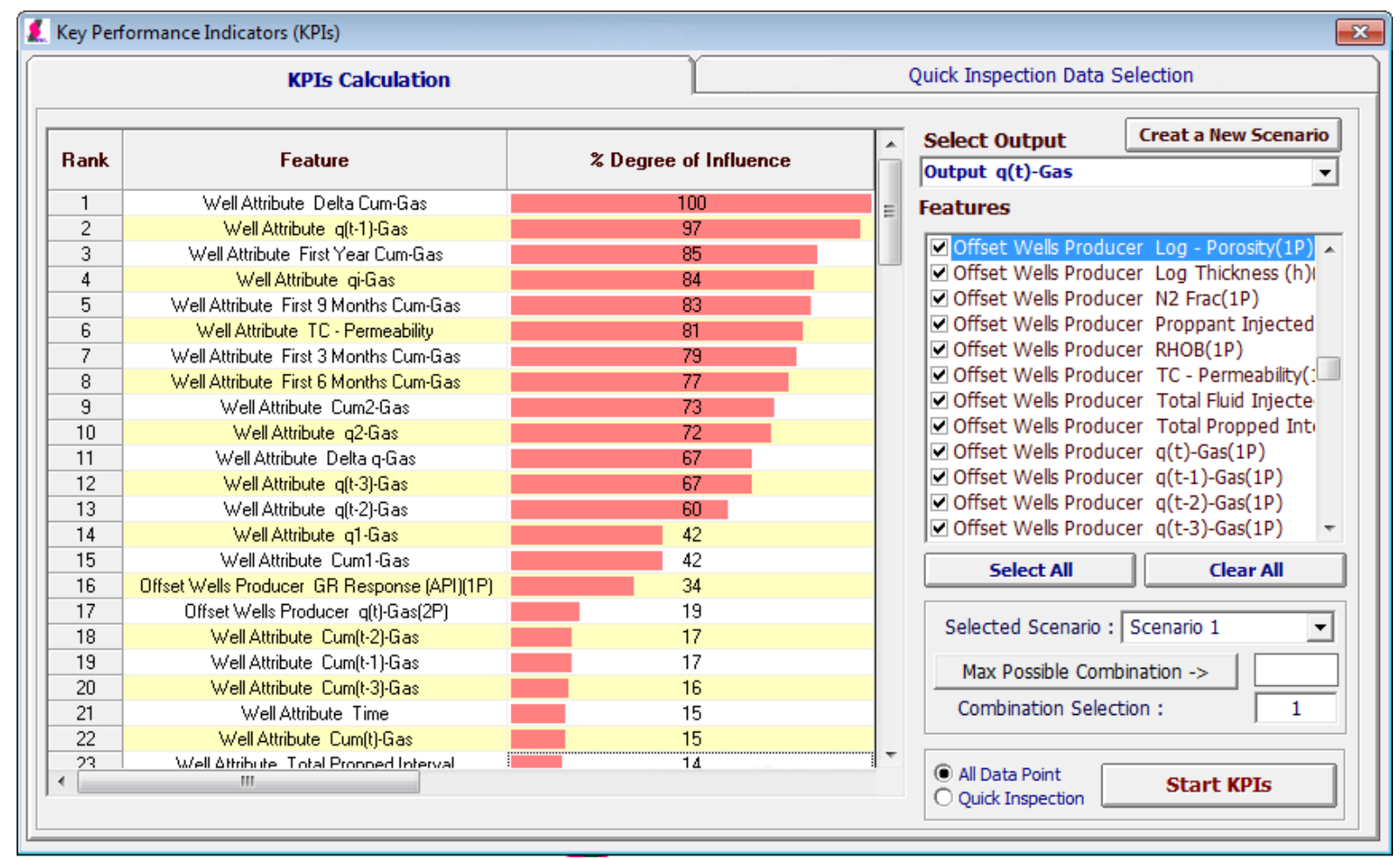

Figure 24 Example of the KPI analysis performed in this study

\subsubsection{Neural Network Training}

Neural network training is in a way a trial and error process. Training starts by selecting input parameters that the developer of the Top Down Intelligent Reservoir Model believes are the most influential on the predicted production rate. Ideally, parameters identified by the KPI analysis as the most influential are selected as inputs for the neural network training. In neural network training procedure imported dataset is parsed in three portions. First portion, which is the largest one, is used for neural network training purposes (providing pairs of inputs and corresponding outputs). Second portion is used for neural network calibration or in other words for tuning the network during the training stage. Finally, third portion of the dataset is used as blind verification dataset. Data contained in verification portion was never proposed to the neural network during the development procedure. Example of parameter selection and neural network verification is shown in figure 25 . 


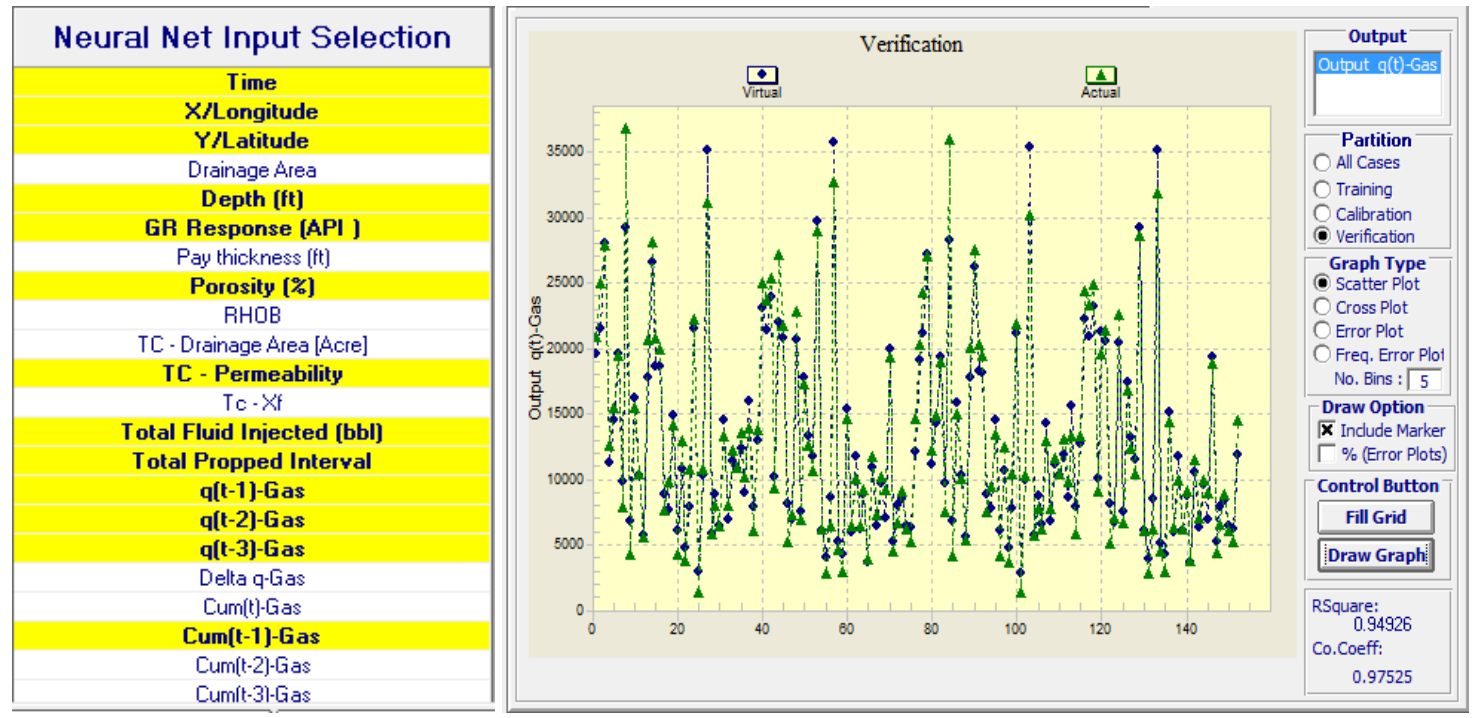

Figure 25 Neural Network selection of inputs (left), and example of training (right)

In Top Down Intelligent Reservoir Modelings predictive model development, three way parsing of the time based database is done in a way such that first portion of the dataset is used for training, second portion for calibration, and very last portion for verification purposes. This approach to database parsing is used due to specific objective of the developed neural network, which is predicting future field performance. And that is the reason why very last portion of the data is selected for verification purposes.

Figure 26 gives schematic description of database parsing used in this study.

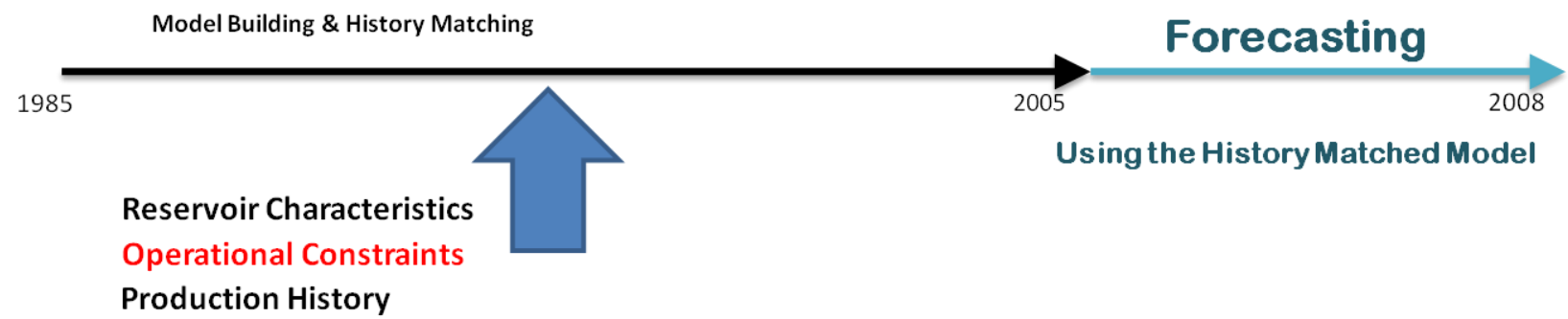

Figure 26 Example of database parsing for neural network training 


\subsection{Model Implementation and Verification}

Final step in predictive model development is model implementation and verification. Neural network trained in previous step is applied in a time successive manner ${ }^{12}$ in order to recreate production profiles of the existing wells within the reservoir. Production profiles generated in this way are plotted against the actual production data in order to verify the accuracy of the developed predictive model. Usually, at the same time future well performance prediction is performed with the developed predictive model and the observed trends are analyzed. If the accuracy of the developed model is within acceptable error range, predictive model development is considered to be completed. Otherwise, the procedure requires going one step back, to the predictive model development stage where different set of inputs is selected for neural network training, and the whole training, implementation and verification procedure repeated.

This process explains the need for very complex dataset whose parts are generated with all the previously described steps of Top-Down Intelligent Reservoir Methodology.

\footnotetext{
${ }^{12}$ Previous time step prediction is used as input for the next time step prediction
} 


\section{RESULTS AND DISCUSSION.}

\subsection{Fuzzy Pattern Recognition (FPR) Analysis}

Production data obtained through single well history matching with Fracgen/Nfflow reservoir simulator was used for FPR analysis rather than real production data, for the sake of increasing accuracy of estimations of reservoir depletion through time, total field recovery, and remaining reserves. In order to accurately estimate reservoir depletion through time, delineation was performed until all scenarios $(3,6,12,36,60,120$ months of production) formed a tornado chart with the same, fixed delineation lines. In this way performed FPR analysis identified portions of the reservoir that gave the most contribution to the field gas production in the first $3,6,12,36$, 60 and 120 months of production.

In figures 27 through 32 results of FPR analysis are shown. Darker boxes correspond to zones of, relatively speaking higher depletion of the reservoir. These results show that the central portion of the reservoir is the most depleted, with somewhat higher depletion in the upper and lower portions of the reservoir. Furthermore, results also indicate that as time progress, the most depleted zone expands towards east and west.

FPR analysis performed on total cumulative production is shown in Figure 33. This analysis also confirmed that middle portion of the reservoir, is relatively speaking the most depleted, with somewhat higher depletion in the upper and lower zones of the reservoir. Figure 34 gives results

of FPR analysis for total gas recovery with the existing wells. Total recovery was calculated based on the fitted decline curves and abandonment rate. 


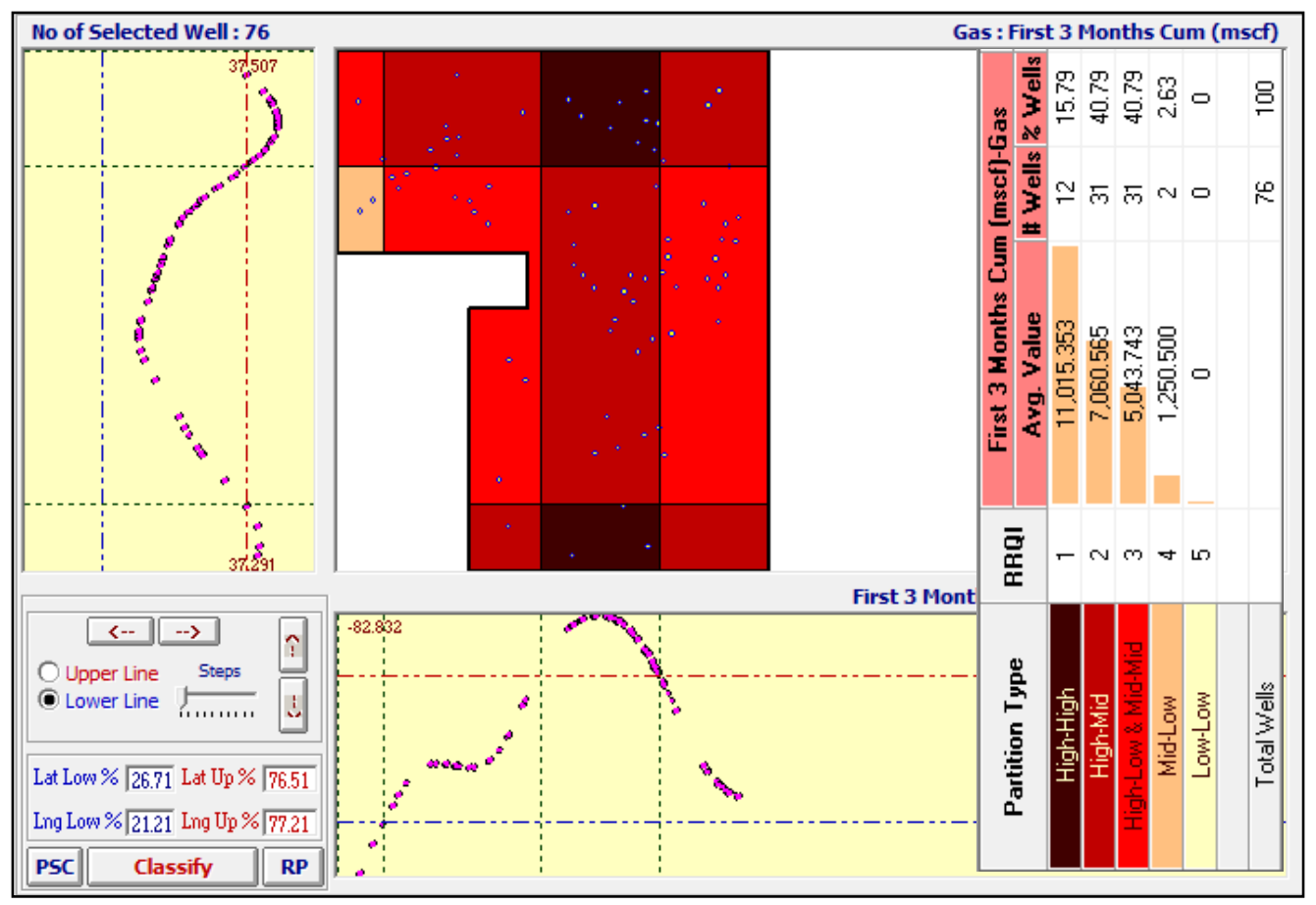

Figure 27 Fuzzy Pattern Recognition Analysis of First 3 Months of Production

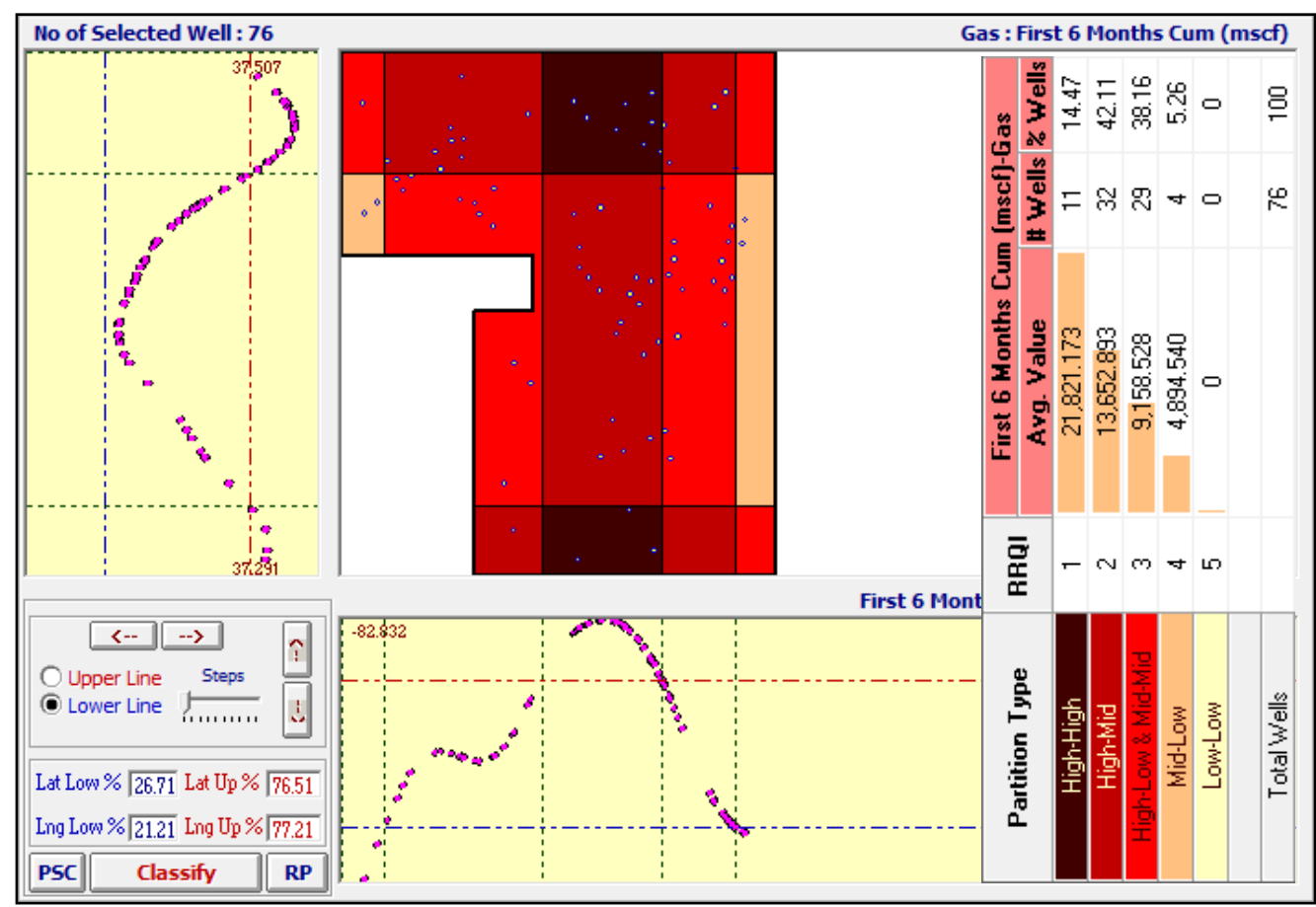

Figure 28 Fuzzy Pattern Recognition Analysis of First 6 months of production 


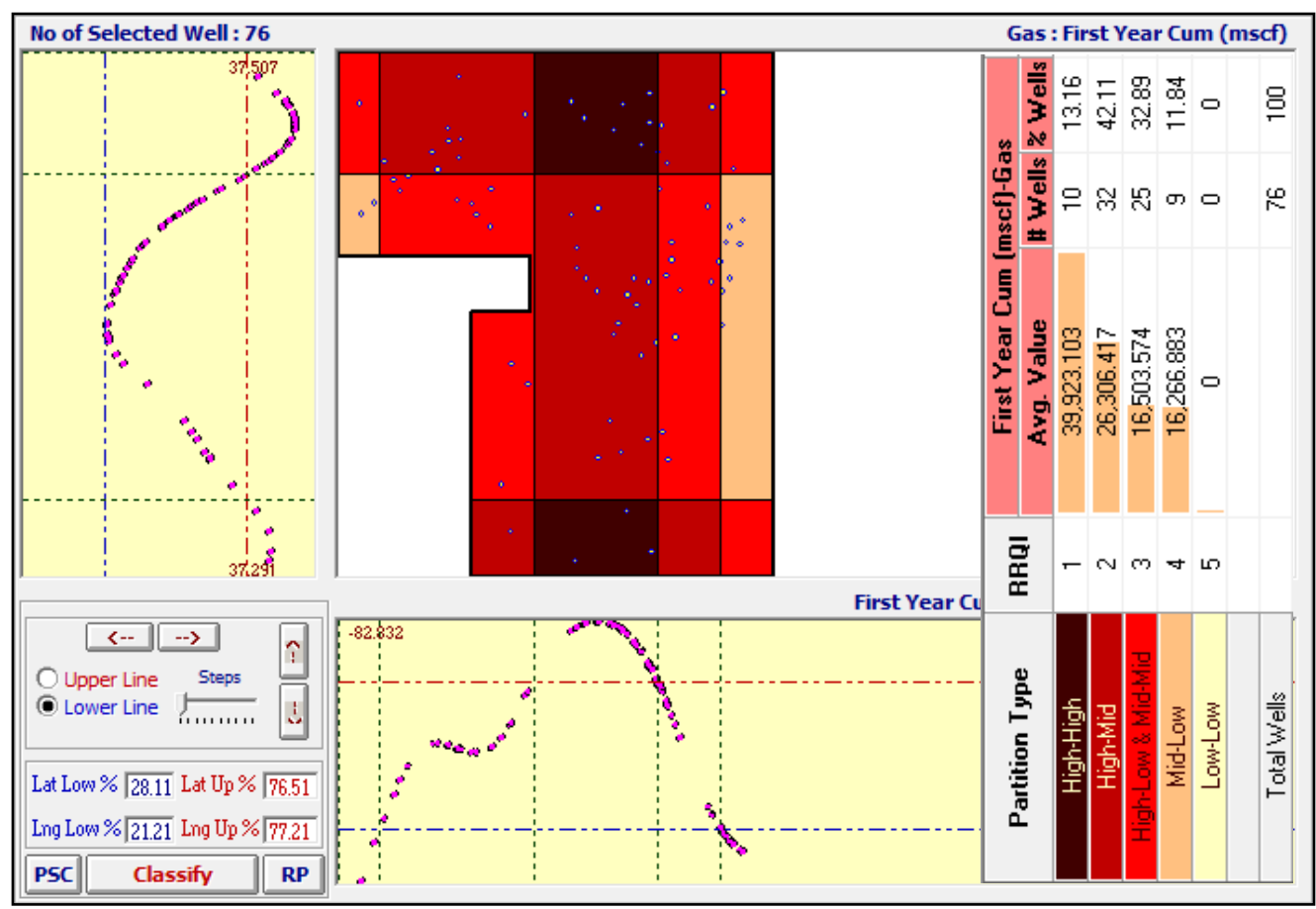

Figure 29 Fuzzy Pattern Recognition Analysis of the first year cumulative production

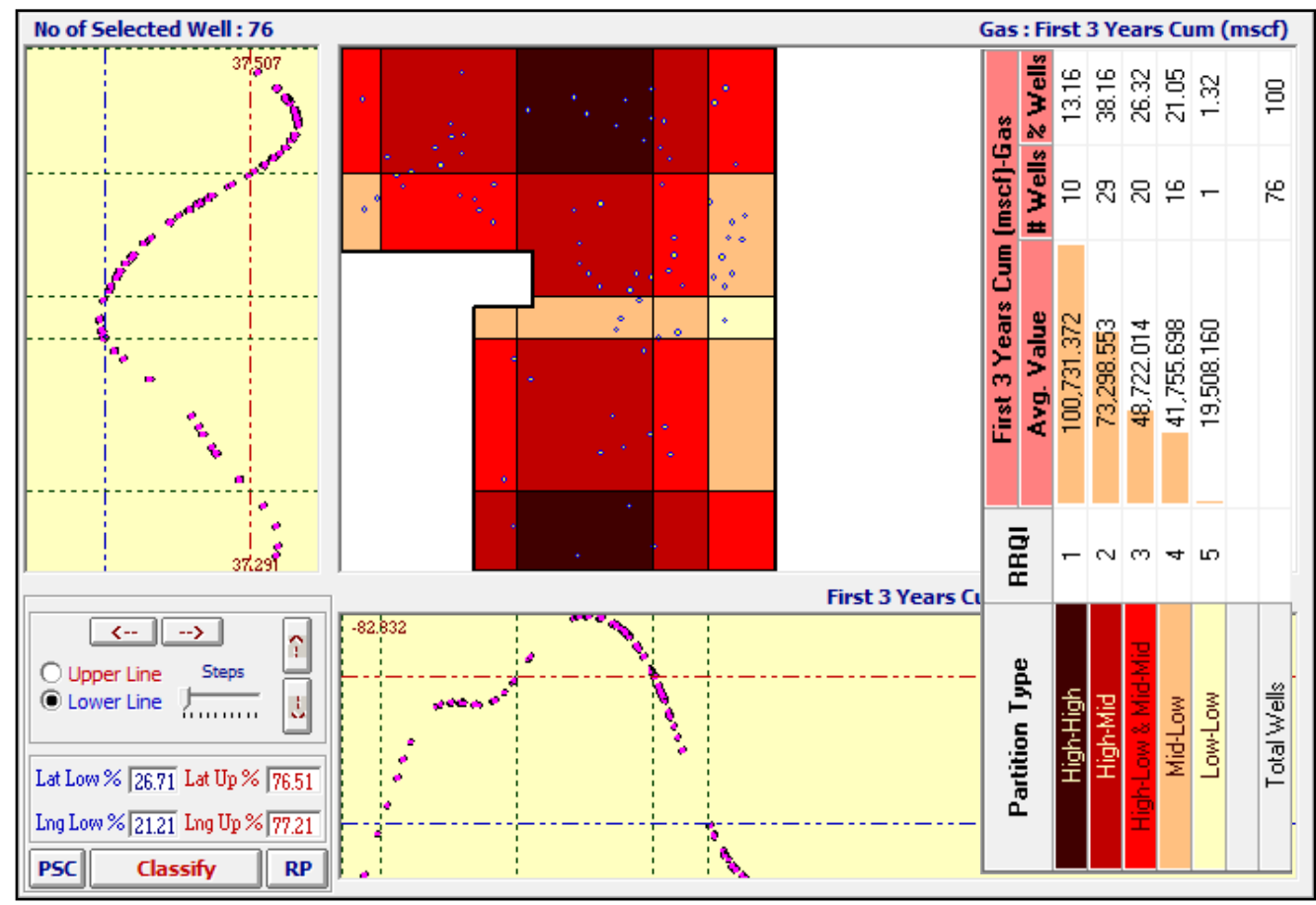

Figure 30 Fuzzy Pattern Recognition Analysis of First 3 years of production 


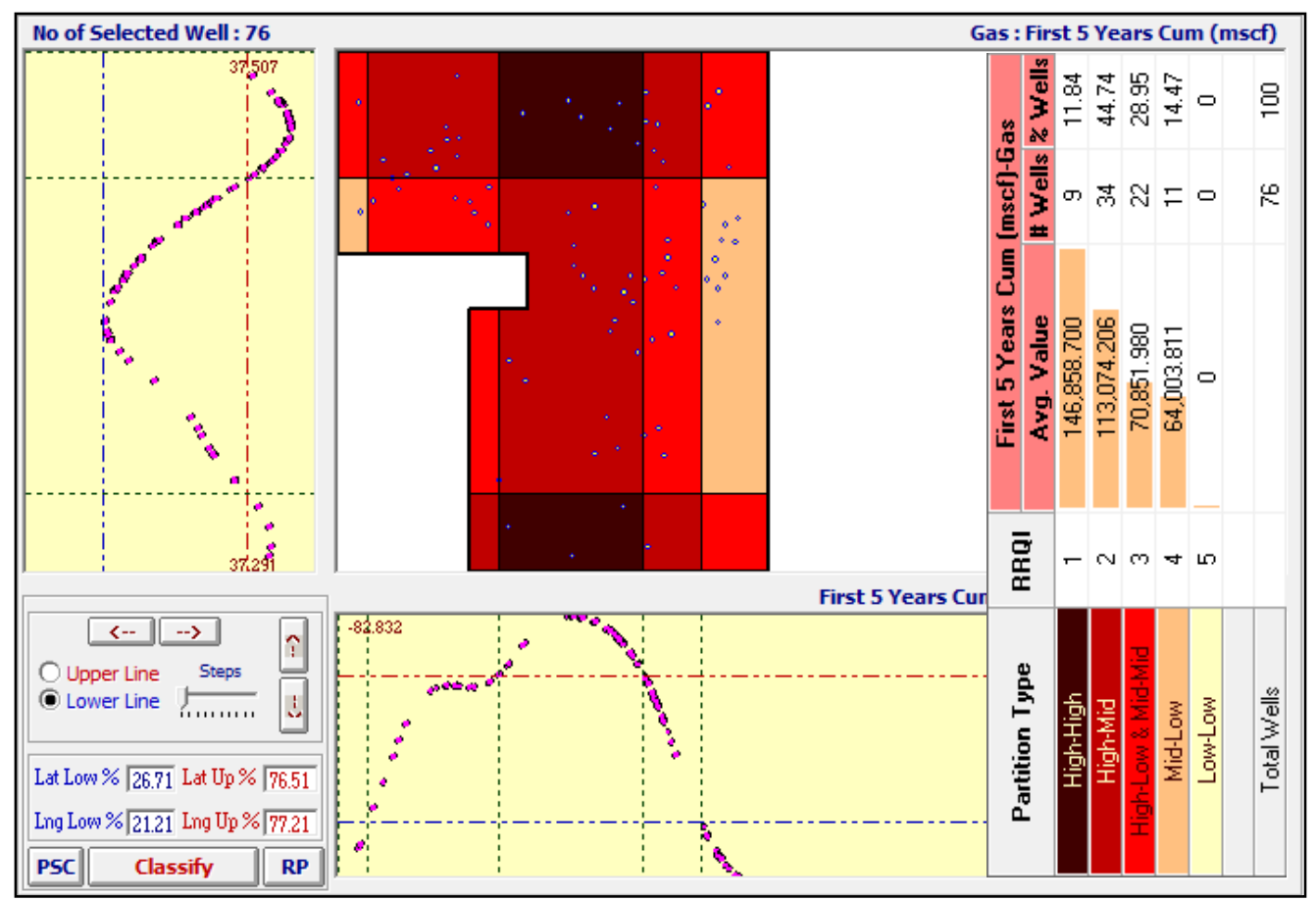

Figure 31 Fuzzy Pattern Recognition Analysis of First 5 Years of Cumulative production

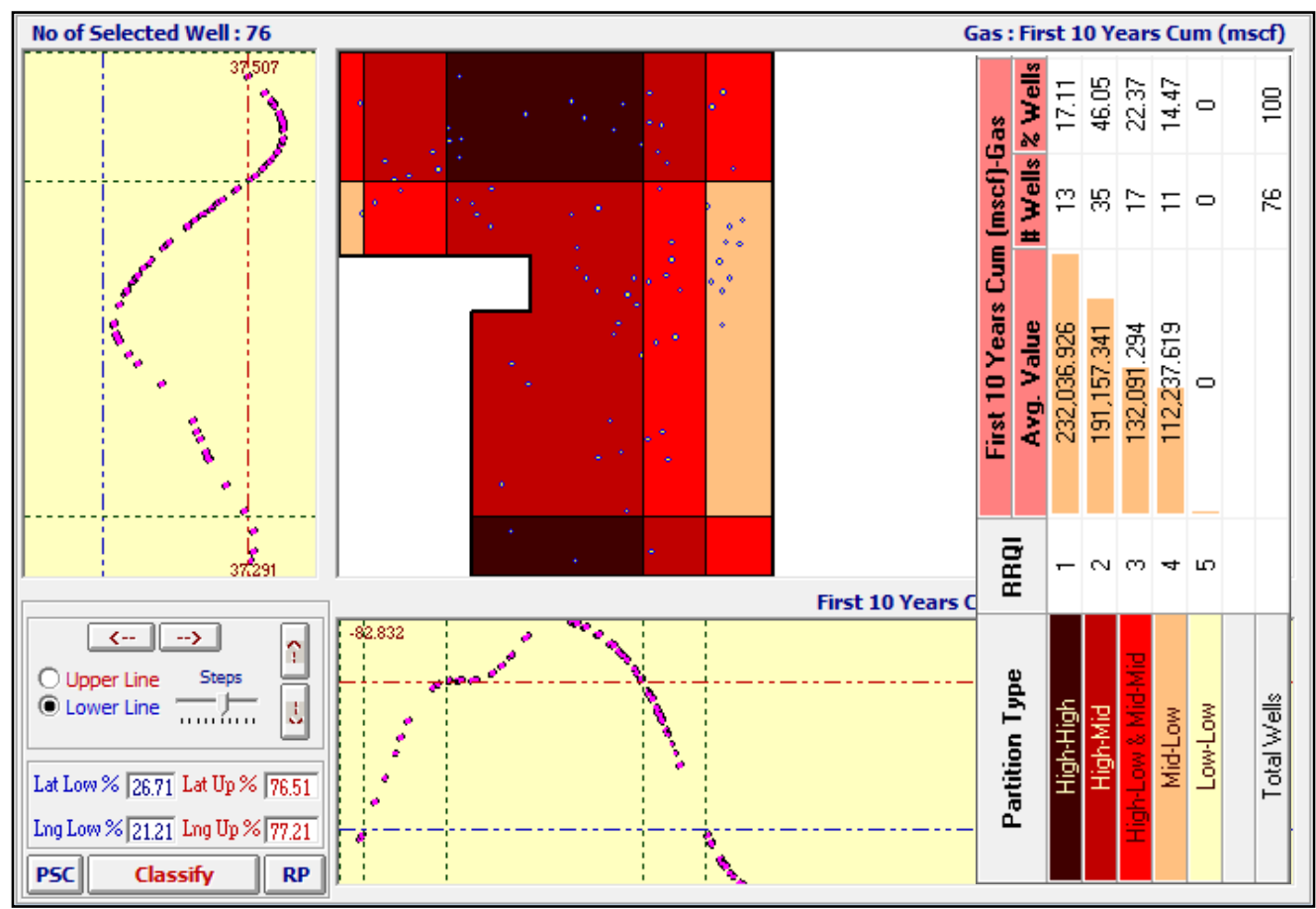

Figure 32 Fuzzy Pattern Recognition Analysis of First 10 Years of Cumulative production 


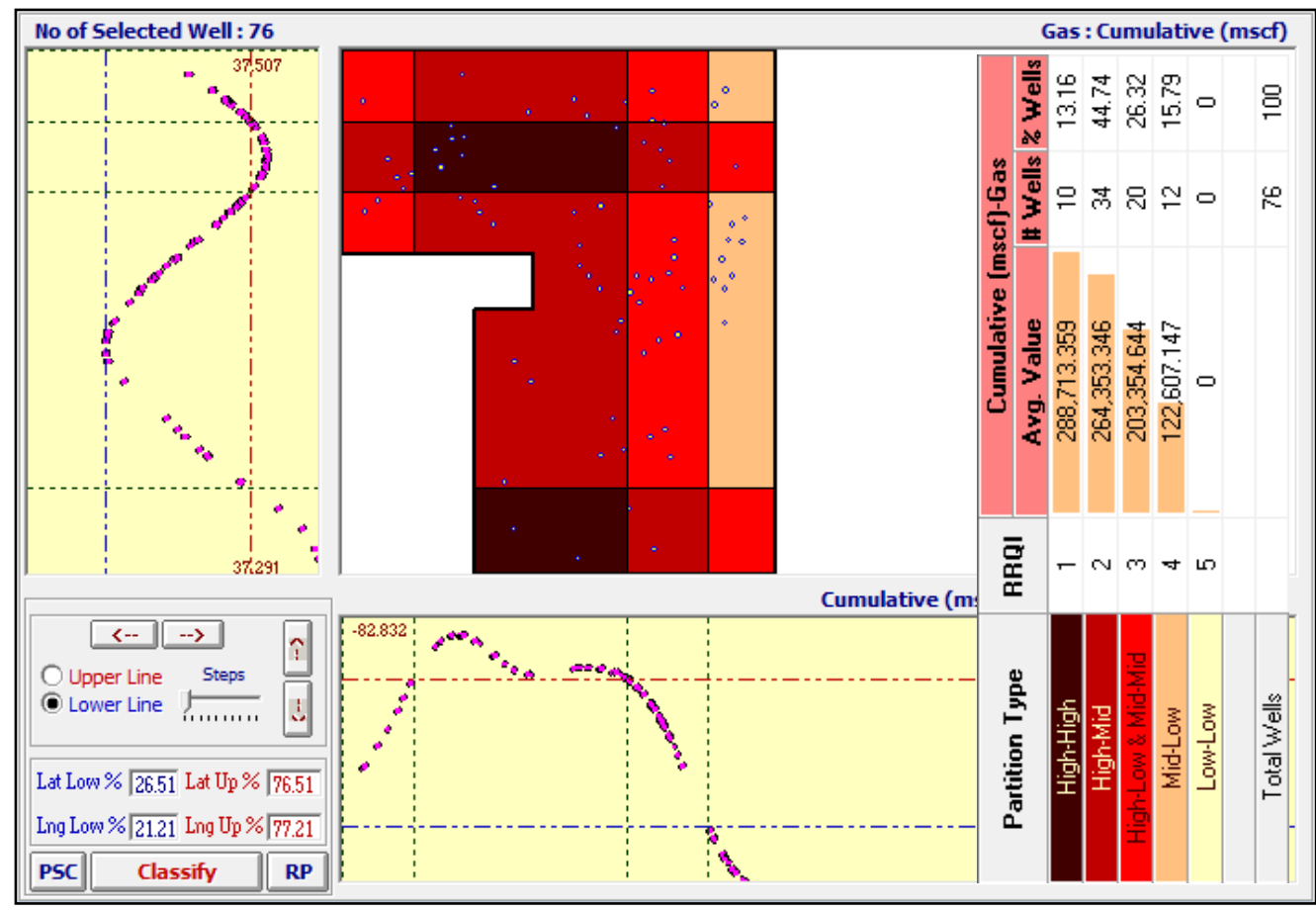

Figure 33 Fuzzy Pattern Recognition Analysis of Cumulative Gas production

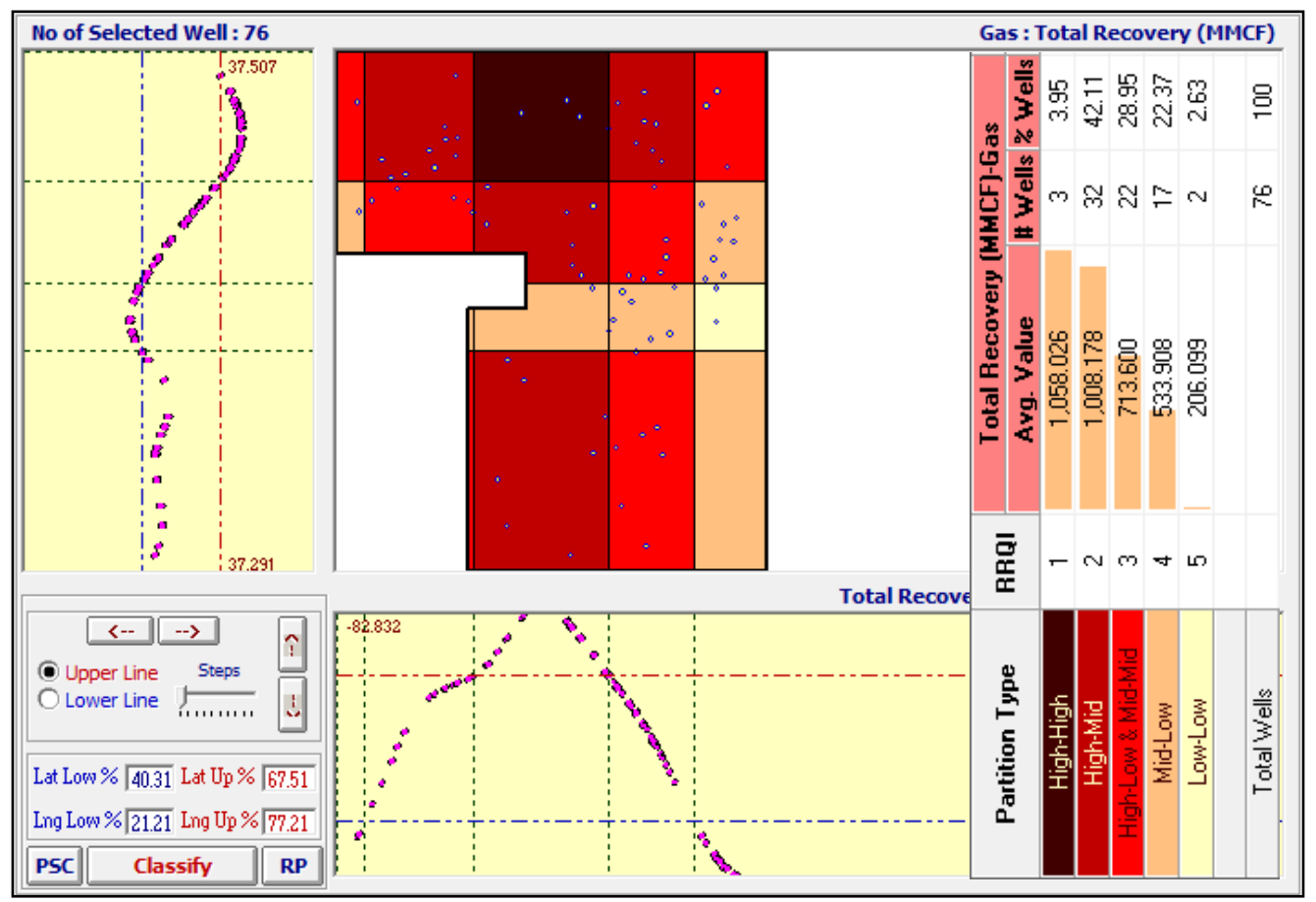

Figure 34 Fuzzy Pattern Recognition Analysis of Total Gas Recovery (with existing wells) 


\subsection{Predictive Modeling Results}

As described in the methodology section, Top Down Intelligent Reservoir Modeling follows certain steps in order to come up with the best dataset to be used in neural network/predictive model development. After KPI analysis was completed, several attempts of neural network training were conducted. In each neural network training attempt, a portion of the database from January 1985 (beginning) to December 2004 was used for training, while portions from January 2005 to December 2006 and January 2007 to December 2008 were used for neural network calibration and verification respectively.

The network that produced the best results was trained with the set of inputs shown in table 3 .

Table 3 Parameters used for final neural network

\begin{tabular}{|c|c|}
\hline Origin & Parameter \\
\hline \multirow{15}{*}{ Well } & Time Step \\
\hline & Well coordinate - Longitude \\
\hline & Well coordinate - Latitude \\
\hline & Depth of the formation at the well ( $f t)$ \\
\hline & Averaged Gamma Ray Reading (API units) \\
\hline & Pay Thickness (ft) \\
\hline & Log Porosity (\%) \\
\hline & Permeability estimated by type curve matching \\
\hline & Total amount of injected fracturing fluid (bbl) \\
\hline & Total propped interval (ft) \\
\hline & Gas rate at previous time step $(t-1)$ \\
\hline & Gas rate at second previous time step $(t-2)$ \\
\hline & Gas rate at third previous time step $(t-3)$ \\
\hline & Cumulative gas production @ ( $t-1)$ \\
\hline & Date of First Production \\
\hline \multirow{3}{*}{ Offset 1} & Distance from the well $(f t)$ \\
\hline & Depth of the formation at the offset well 1 \\
\hline & Gas rate of the offset well 1 at $(t-1)$ \\
\hline \multirow{2}{*}{ Offset 2} & Distance from the well $(f t)$ \\
\hline & Gas rate at offset well 2 at $(t-1)$ \\
\hline
\end{tabular}


Figure 35 shows cross plot of the trained neural network. In ideal conditions cross plot of neural network predicted values vs. real values provided for training, should yield to a straight line. Considering the fact that we were dealing with the real data, cross plot in figure 35 is an example of an overall nicely trained neural network.

After neural network development was completed, same network was applied in order to generate full production profiles both of single wells, and full field production profiles. Figure 36 shows actual full field production rate vs. predictive model generated full field production rate. The same figure also includes prediction of the full field production rate for the following 7 years, from the time of last database entry. As figure 36 indicates, predictive models prediction and general field production rates trend are in overall very good agreement.

Following figure 36 are examples of single well real production profiles vs. the predictive model generated production profiles. Overall, results of the predictive model appear to be in good agreement with the real data. Predictive modeling results for all wells used in this study are provided in Appendix-B. 


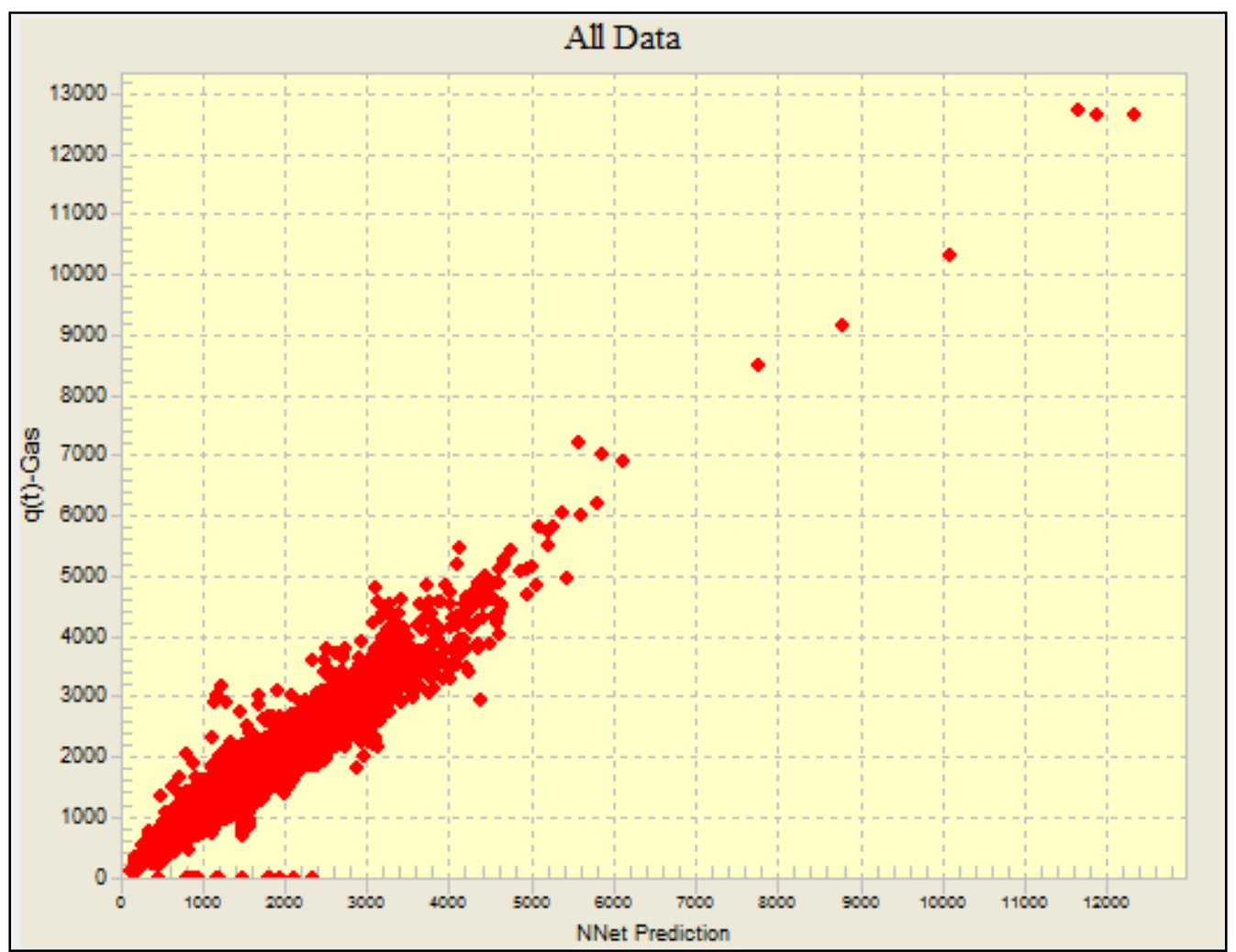

Figure 35 Neural Network Training Results (Y Axis - Real Data, X Axis -Neural Network Predicted Values)

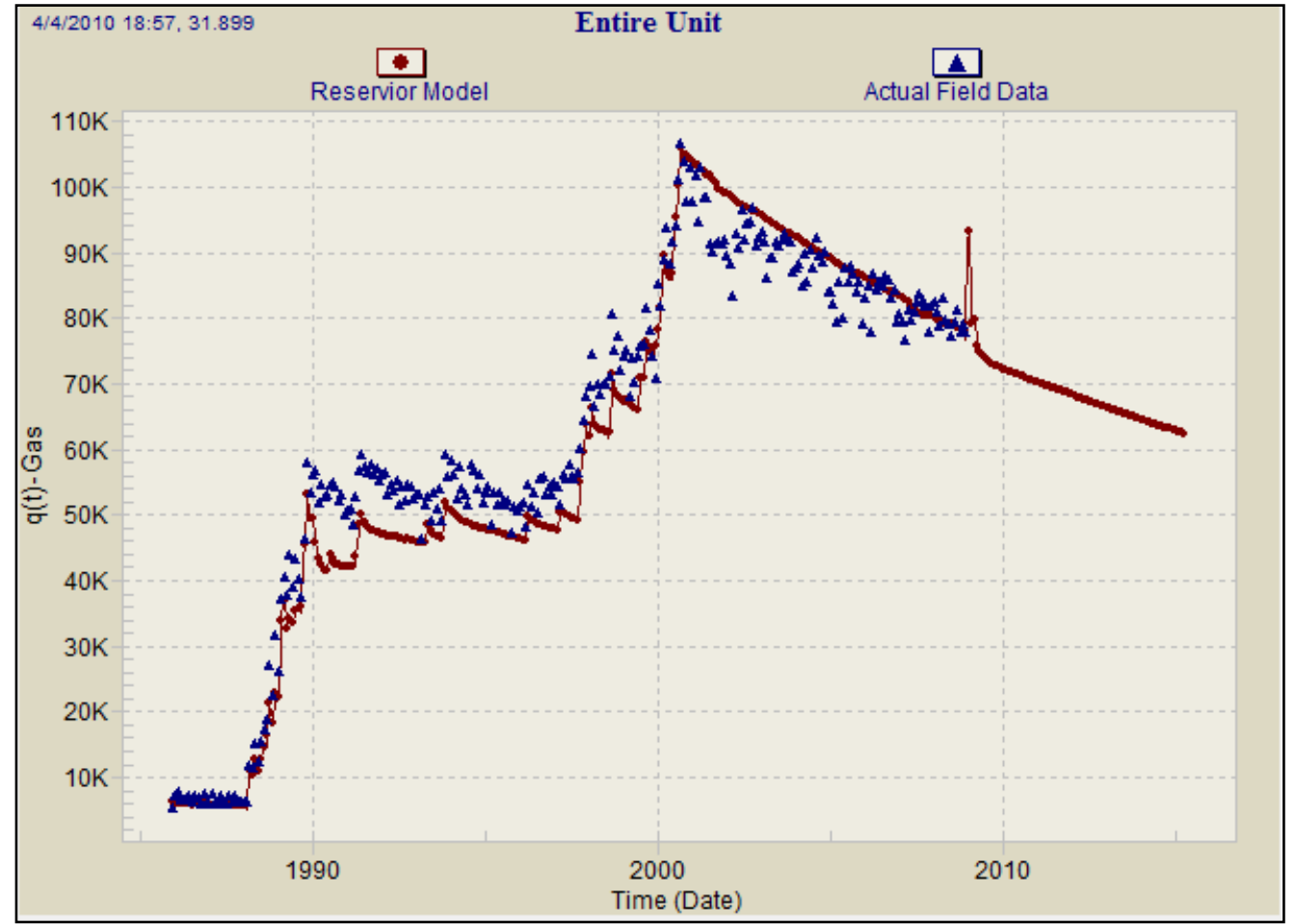

Figure 36 Real field production rate vs Top Down predictive models field production rate 


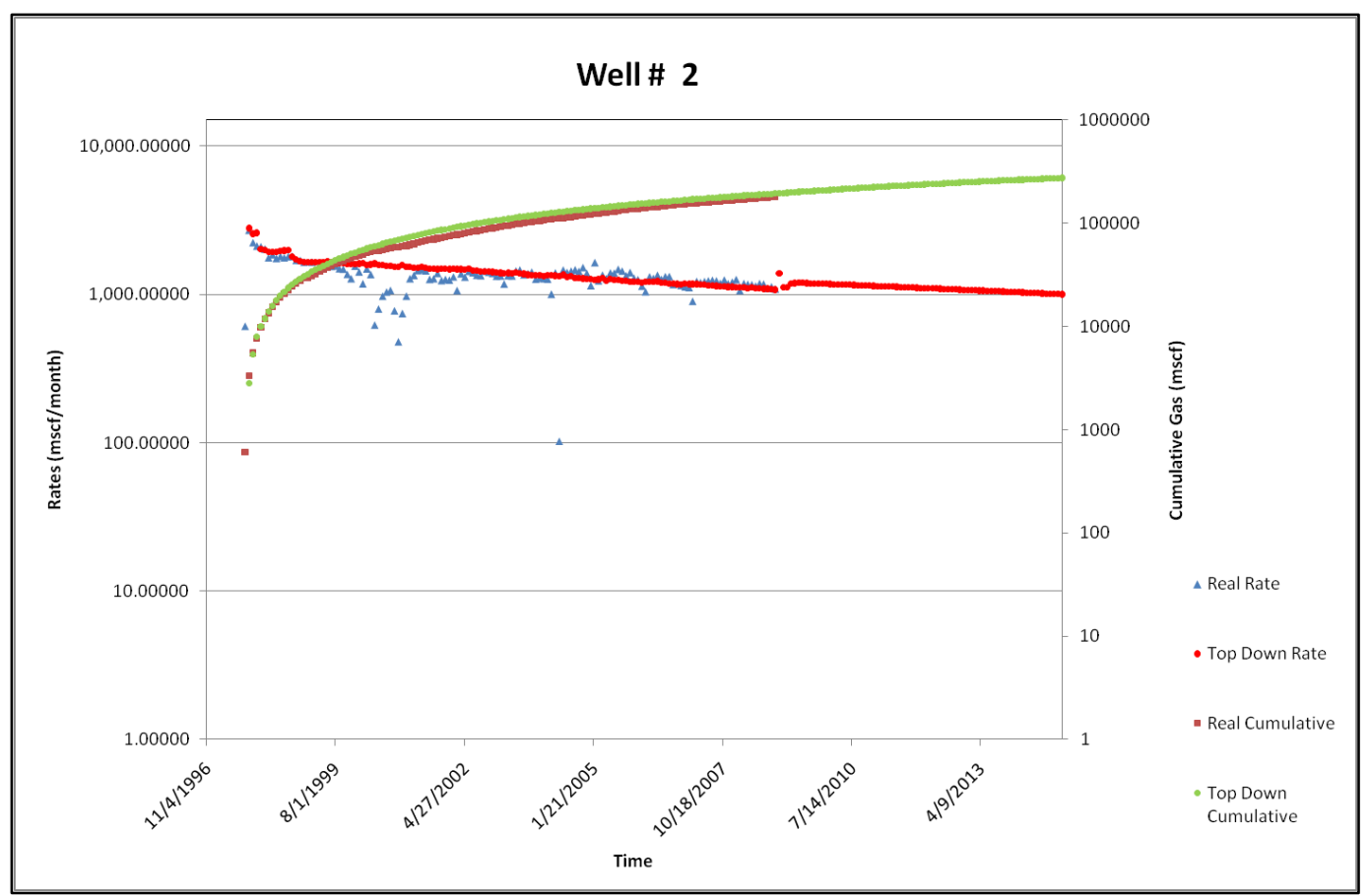

Figure 37 Example of Top-Down predictive model results for a single well

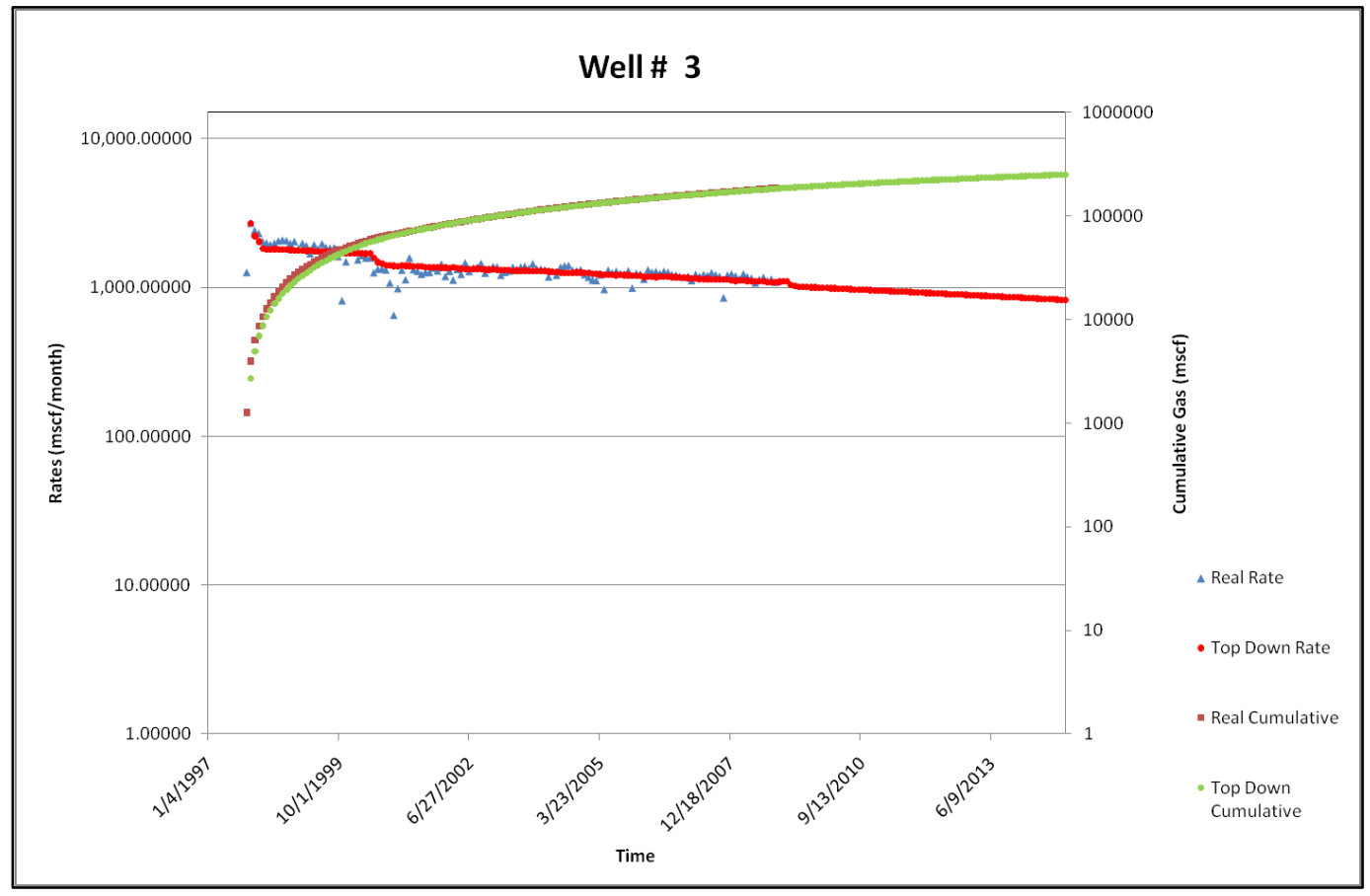

Figure 38 Example of Top-Down predictive model results for a single well 


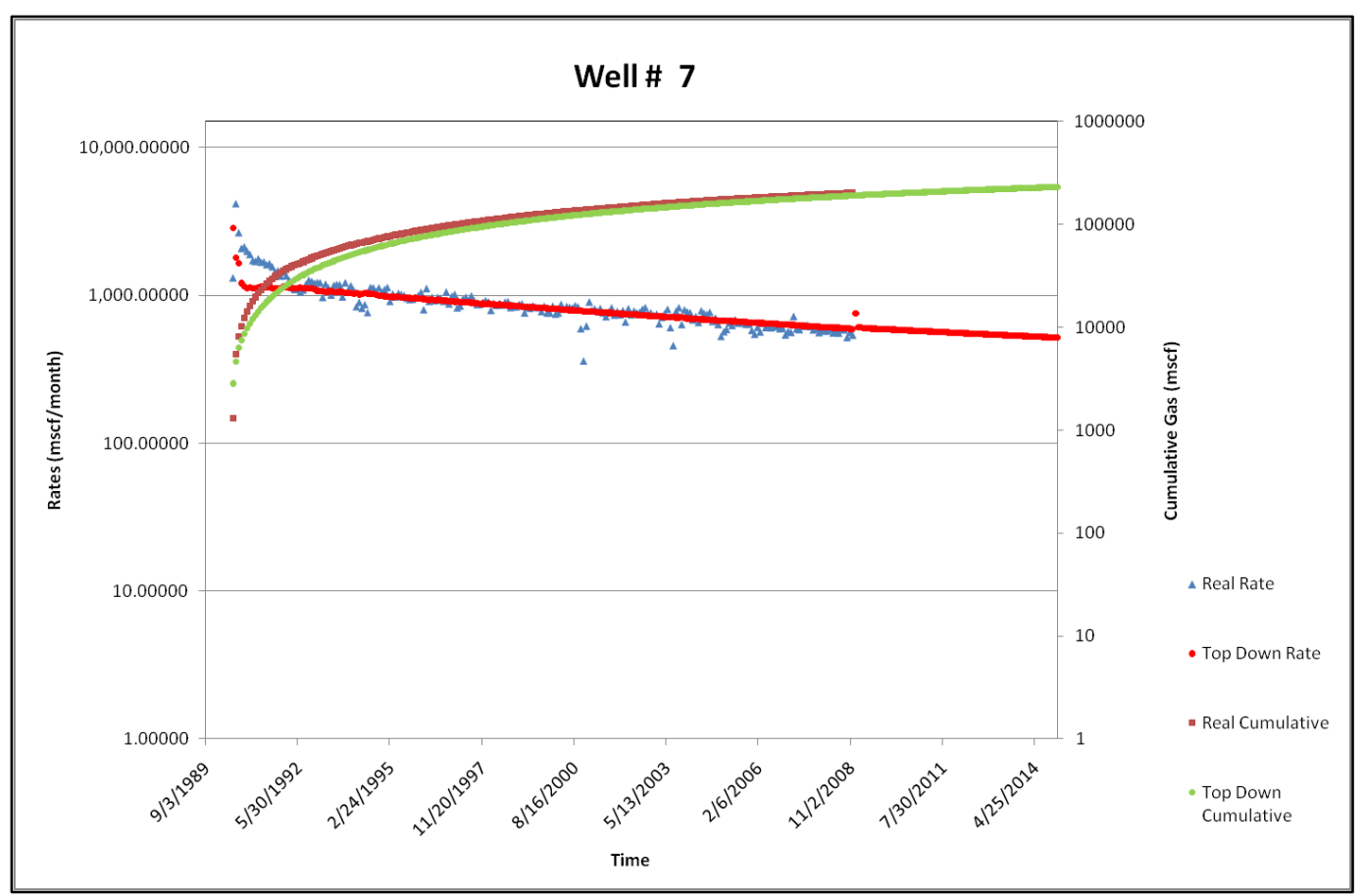

Figure 39 Example of Top-Down predictive model results for a single well

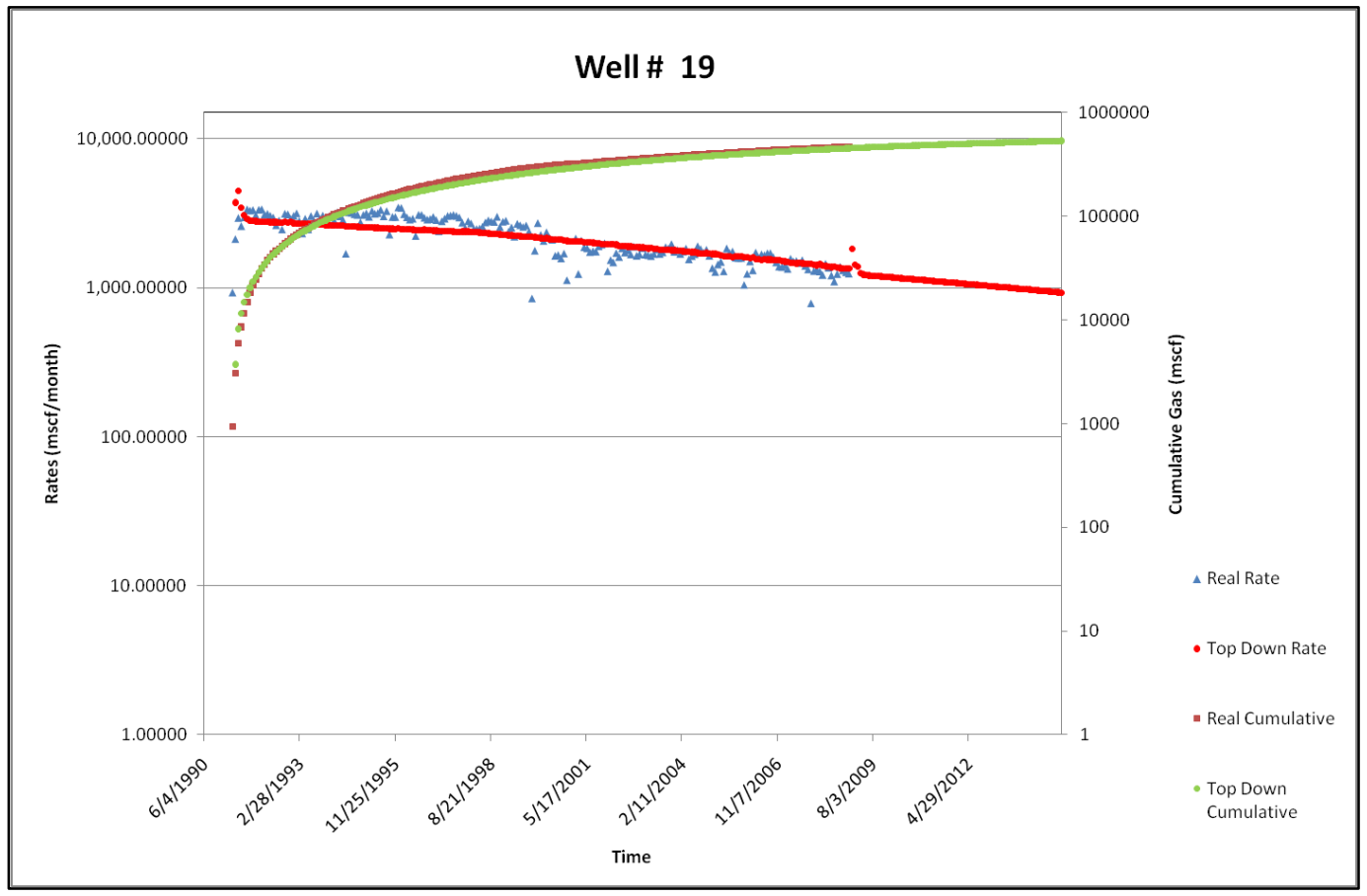

Figure 40 Example of Top-Down predictive model results for a single well 


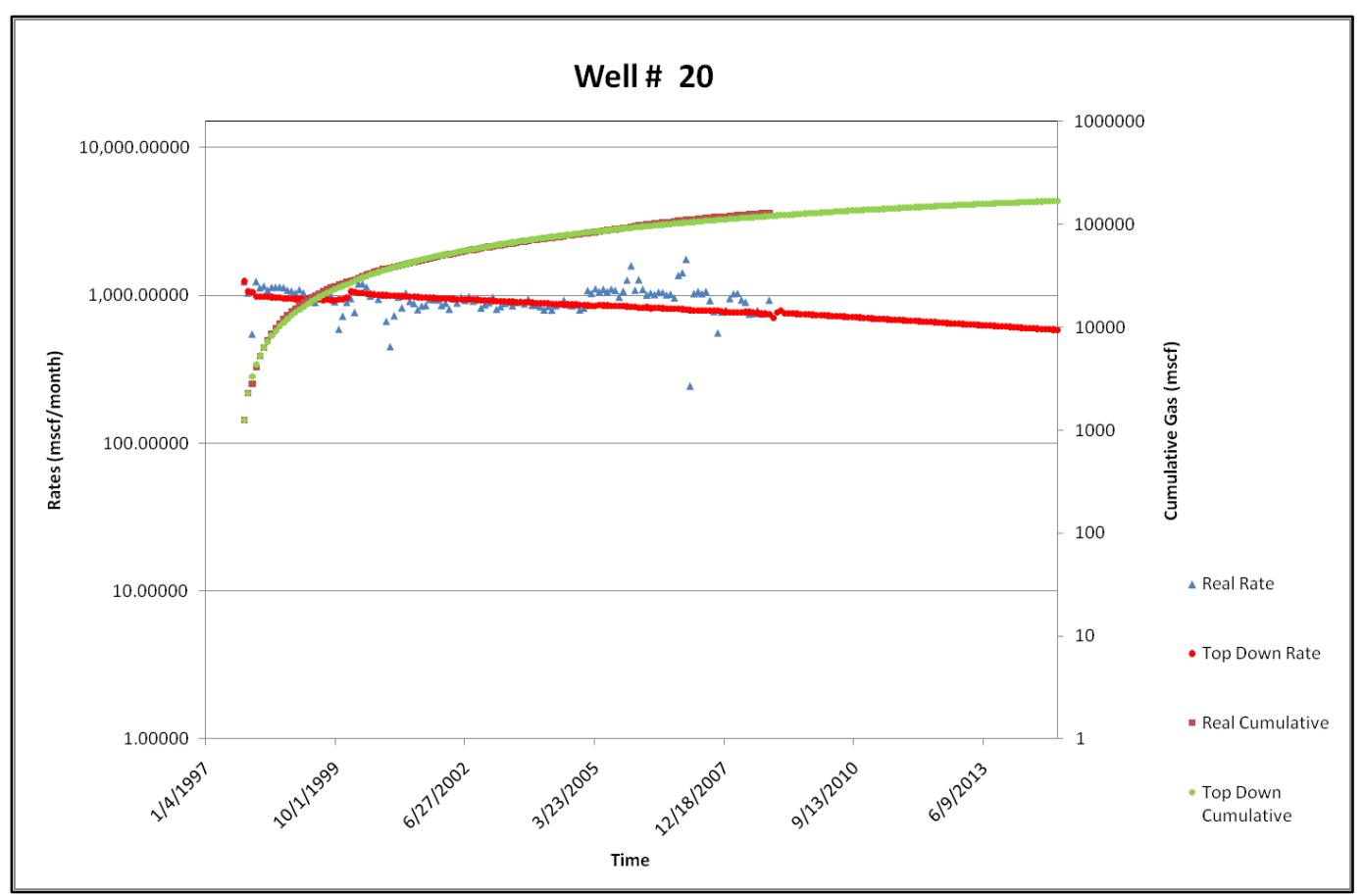

Figure 41 Example of Top-Down predictive model results for a single well

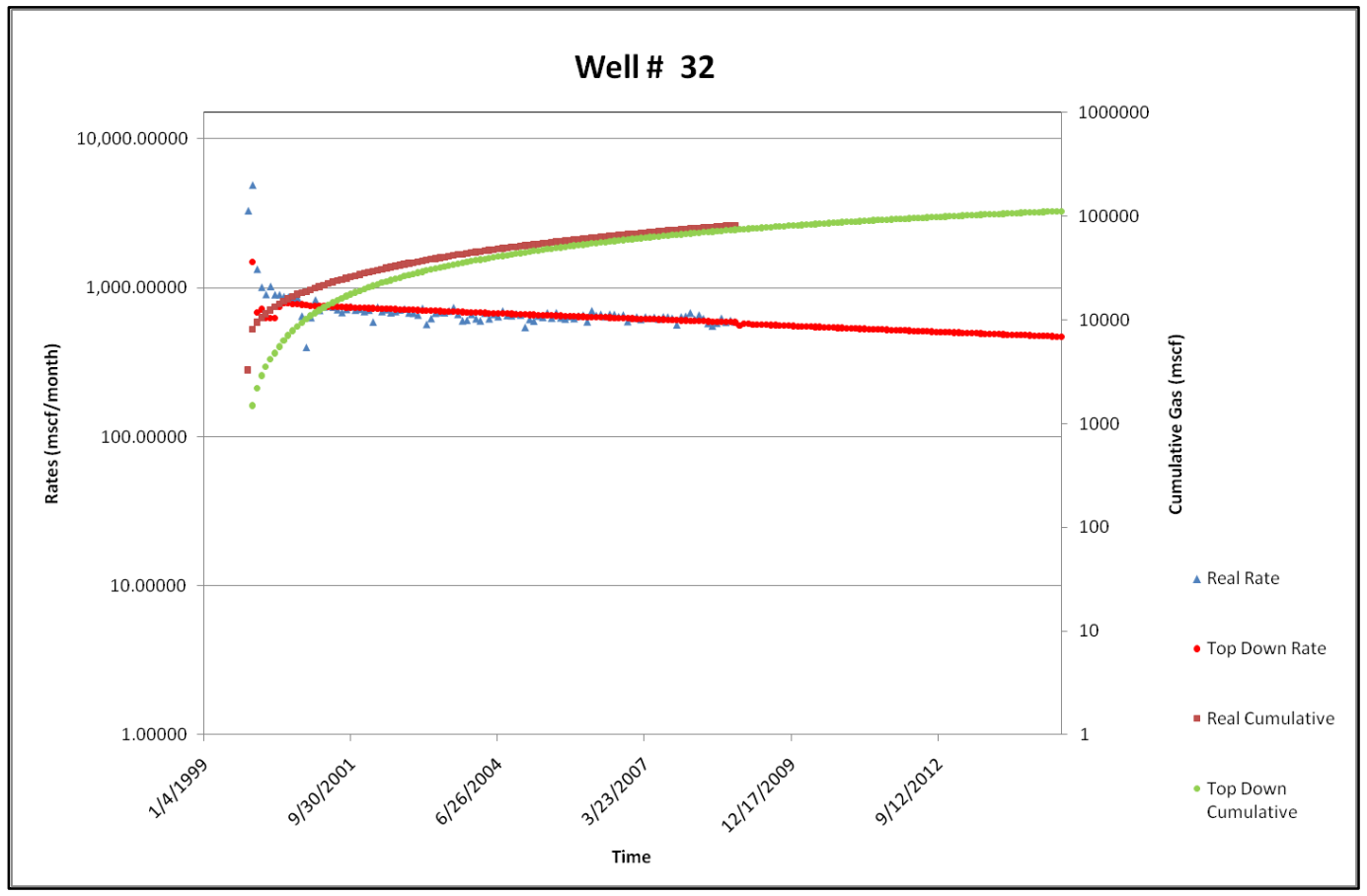

Figure 42 Example of Top-Down predictive model results for a single well 


\subsection{Predictive Modeling - Sensitivity Analysis}

Development of the predictive models by employing neural networks enables very fast sensitivity analyses. Influences of two or more parameters are very quickly estimated by simply changing the values and observing neural network produced performance curves. Sensitivity analysis of the developed predictive model was performed in order to observe general model behavior influenced with a combination of two input parameter.

Analyses presented here were performed in order to estimate overall influence of stimulation jobs parameters (hydraulic fracturing) on gas production rate. Figure 43 represents sensitivity analysis for the influence of propped interval and log porosity on overall gas production rate. Figure 44 was produced for the influence of total stimulated interval and total amount of injected fluid on the overall production rate. Figure 45 was produced for the influence of Lower Huron shales thickness (Pay Thickness) on overall production rate.

Based on figures 43 and 44 , the most efficient fracturing jobs were performed by fracturing between 350 and $450 \mathrm{ft}$ of Devonian shale thickness (both Lower Huron Shale and "Transition zone"), and by injecting between $6500-9500$ gallons of water. 


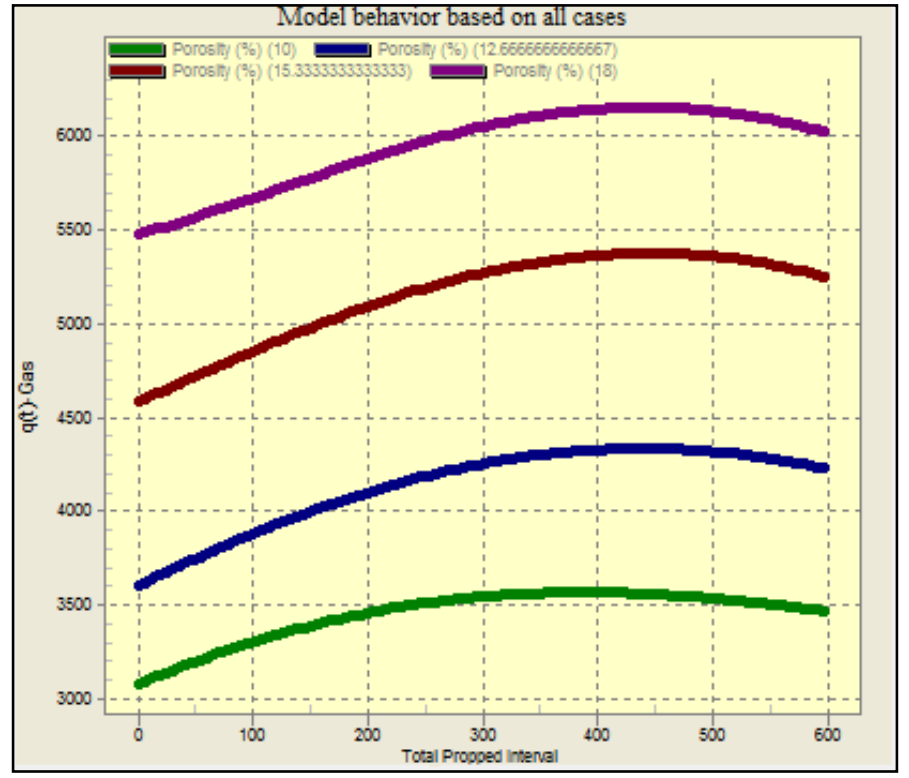

Figure 43 Predictive models sensitivity analysis

(Production vs. Total propped interval for different Log Porosity values)

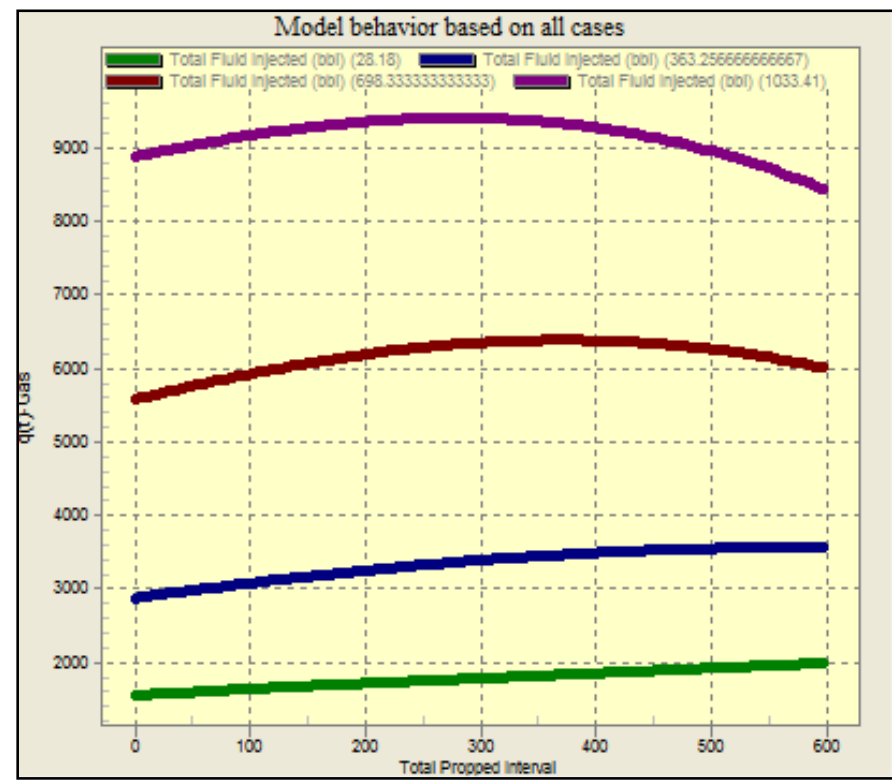

Figure 44 Predictive models sensitivity analysis

(Production vs. Total propped interval for different amounts of injected propping fluid) 


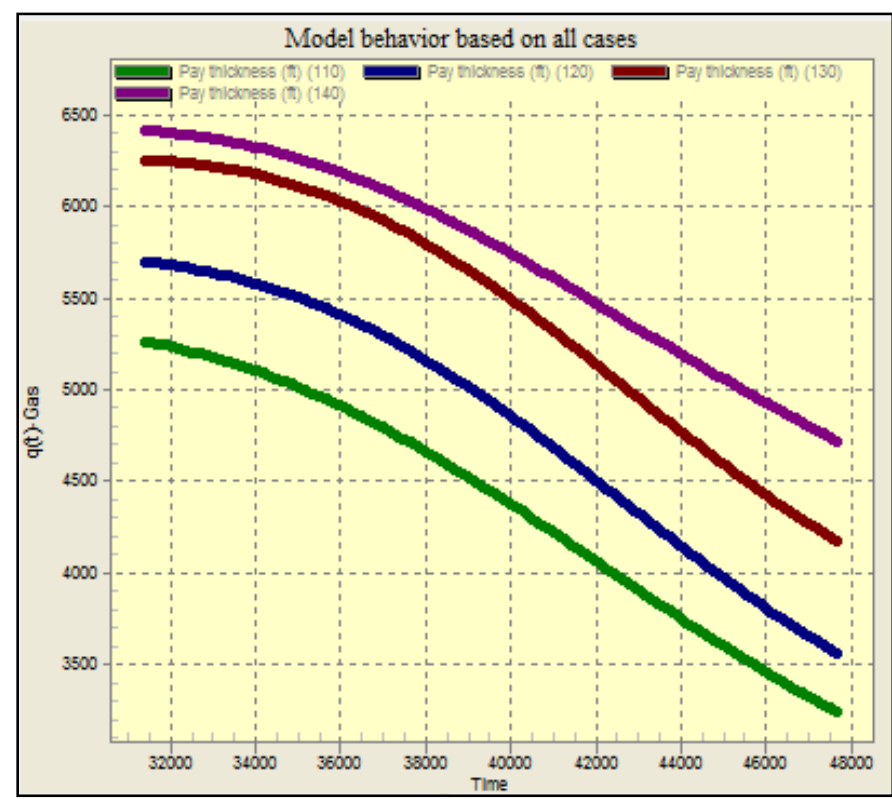

Figure 45 Predictive models sensitivity analysis

(Production vs. Time for different values of pay thickness) 


\section{CONCLUDING REMARKS \& FUTURE WORK}

\subsection{Conclusion}

This study demonstrated high applicability of the Top-Down Intelligent Reservoir Methodology to shale gas reservoir modeling and production data analysis. One of the main advantages in using this methodology for shale gas reservoir simulation and modeling is its ability to analyze the reservoir from qualitative, rather than quantitative point of view which appears to be the most suitable method of analyzing highly uncertain shale gas reservoirs. Another advantage, again from the point of shale gas modeling is that the usage of artificial neural networks in modeling these reservoirs seems to be the most appropriate method, due to not fully established functional relationships between the acquirable reservoir parameters and the actual production data. For example it has been known for a long time that the most productive areas of the shale gas reservoirs had higher organic content, which in turn increased their radioactivity, manifesting in high Gamma Ray (GR) well log readings. Therefore, the relationship between GR readings and productivity was evident. The only method known so far, capable of incorporating such geophysical data into reservoir simulation and modeling is Top-Down Intelligent Reservoir Modeling methodology. That is exactly where the true power and very bright future for this method, in shale gas reservoir modeling and simulation lies.

It is important to mention that overall very good results obtained in this study did not incorporate reservoir pressure, gas sorption and operational constraints data. This data was most probably inferred by the neural network during the history matching/training procedure, and it constitutes the model in a form of adjusted weights of synaptic neural network connections. However, the accuracy of the model and future prediction capabilities, in terms of different operational 
scenarios, would be much better if operational constraints have been provided to the network in the development phase.

Finally, Top Down Intelligent Reservoir Modeling represents a very innovative approach to the reservoir modeling in general, and most probably the best technique nowadays available, for shale gas modeling.

\subsection{Future work recommendations}

Future work on Lower Huron Shales Top-Down Intelligent Modeling should focus on incorporating more data about operational constrains, and extend the analysis to a much larger area by including more wells into the analysis. Lessons that can be learned in Top Down Intelligent Reservoir modeling of such highly developed shale gas field, would contribute to the future Top Down Intelligent Reservoir Modeling applications to other shale gas plays in the development, such as for example Marcellus shale. 


\section{BIBLIOGRAPHY}

[1] Steven W. Poston \& Bobby D. Poe (2008), Analysis of Production Decline Curve, Society of Petroleum Engineers.

[2] Schepers K. C., Nutall B., Oudinot A. Y., Gonzalez R., (2009). Reservoir Modeling and Simulation of the Devonian Gas Shale of Eastern Kentucky for Enhanced Gas Recovery and CO2 Storage, SPE 126620 (p. 20). Annual 2009 SPE International Conference on Capture, Storage, and Utilization, San Diego, California

[3] Cox D.O., Kuuskraa V.A., Hansen J.T. (1996), Advanced Type Curve Analysis for Low Permeability Gas Reservoirs, SPE 35595 (pg. 14). 1996 SPE Gas Technology Conference, Calgary, Alberta

[4] Kubik, W. T. (1993), Characterization of Natural Fractures in the Devonian Shale, GRI Experimental Development Research Area, Pike County, Kentucky, 1993 Gas Research Institute, Chicago, Illinois

[5] McKoy, M. S. (March, 1997). Tight Gas Reservoir Simulation: Modeling Discrete Irregular Strata-Bound Fracture Networks and Network Flow, Including Dynamic Recharge from the Matrix, Proc. DOE Natural Gas Conference. Houston

[6] Mohaghegh, S. D. (2009). Top Down Intelligent Reservoir Modeling (TDIRM); A new approach in Reservoir Modeling by Integrating Classic Reservoir Engineering With Artificial Intelligence \& Data Mining, (p. 10). 2009 AAPG Annual Convention, Denver Colorado:

[7] Mohaghegh, S. D. (2011). Reservoir Simulation and Modeling Based on Pattern Recognition, SPE 143179 (p. 13)., 2011 SPE Digital Energy Conference and Exhibition, Woodlands Texas

[8] Mohaghegh, S. D. (September, 2000). Virtual Intelligence applications in petroleum engineering - Part 1: Artificial neural networks, Journal of Petroleum Technology, Distinguished Autor Series, 64-73.

[9] Ning X., F. J. (1993). Measurement of shale matrix and fracture properties in naturally fractured cores using pusle testing, Gas Shales Technology Review, v8, no.2, 34-45.

[10] Grujic O., Mohaghegh S. D., Bromhal G. (2010). Fast Track Reservoir Modeling of Shale Fromations in the Appalachian Basin. Application to Lower Huron Shale in Eastern Kentucky, SPE 139101 (p.11), 2010 SPE Eastern Regional Meeting Morgantown, West Virginia: SPE.

[11] Boswell R., (1996). Upper Devonian Black Shales, Atlas of Major Appalachian Gas Plays, DOE, Gas Research Institute, WV Geological Survey (93-99). 
[12] Mohaghegh S.D., Grujic O., Zargari S., Kalantari M. (2011). Modeling, History Matching, Forecasting and Analysis of Shale Reservoirs Performance Using Artificial Intelligence, SPE 143875 (pg. 12), 2011 SPE Digital Energy Conference and Exhibition, Woodlands Texas

[13] Mohaghegh S.D., Bromhal G., (2010). Top-Down Modeling: Practical, Fast Track, Reservoir Simulation \& Modeling for Shale Formations, (pg. 4). AAPG/SEG/SPE/SPWLA HEDBERG CONFERENCE, Austin, Texas: AAPG/SEG/SPE/SPWLA.

[14] Various. (2009, March). Wikipedia Online Free Encyclopedia. Retrieved April 2011, from: Artificial Neural Networks: http://en.wikipedia.org/wiki/Artificial_neural_network,

[15] Various. (2010, April). Wikipedia Online Free Encyclopedia. Retrieved April 2011, from: http://en.wikipedia.org/wiki/Nervous_system,

[16] Khazaeni Y., Mohaghegh S.D. (2010), Intelligent Time Successive Production Modeling, SPE 132643 (p. 17). 2010 SPE Western Regional Meeting, Anaheim, California

[17] Gomez Y., Khazaeni Y., Mohaghegh S. (2009), Top Down Intelligent Reservoir Modeling (TDIRM), SPE 124204 (p. 11)., 2009 SPE Annual Technical Conference and Exhibition, New Orleans, Louisiana. 


\section{APPENDIX - A}

This chapter gives a short description about the WVU-FGI software application developed as an interface for Fracgen - Natural fracture network generating software. Motivation for the development of such application came from a need for quick manipulation of input parameters required by Fracgen for generating natural fracture network representations, and automatically visualizing generated fracture network. Originally this was supposed to be performed by tedious editing of textual input files and executing two separate applications.

Figure 46 shows initial input screen of WVU-FGI application. At this stage of fracture network modeling user is required to provide information about reservoir extents, and model type of the current fracture set. Fracture set types used by the Fracgen are described in detail in the Fracgen/Nfflow user manual.

Figure 47 is input screen where orientation of the fractures within the current set is provided to the software. Automatically as values are entered rose diagram to the right is updated to show fracture orientation and entered deviation.

Figure 48 shows fracture properties input screen, where user is able to control fracture distribution of the current fracture set, fracture aperture, and minimum and maximum fracture length.

Figures 49 and 50 show visualization of the generated fracture network, and well input screen respectively. 


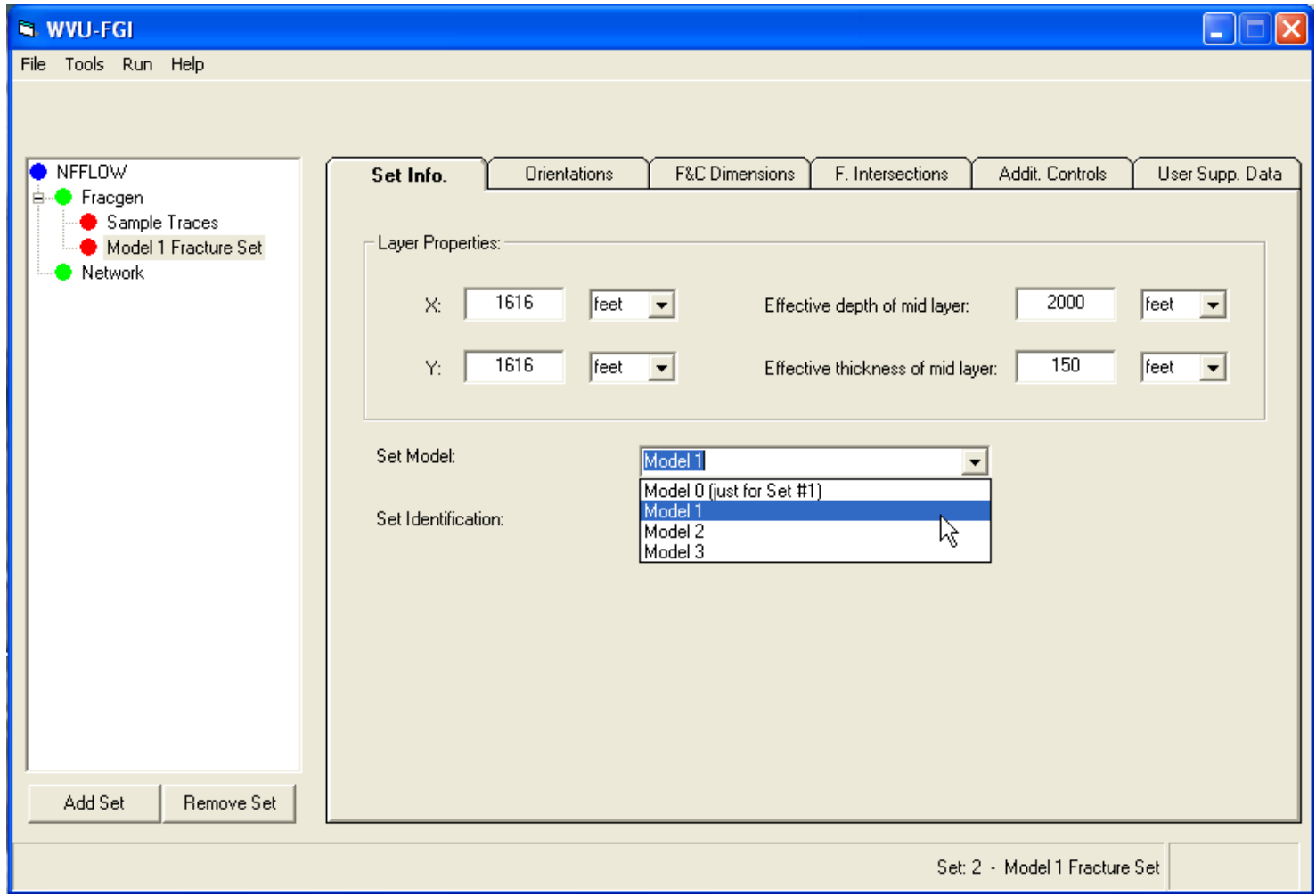

Figure 46 WVU - FGI Fracture Set Selection Screen

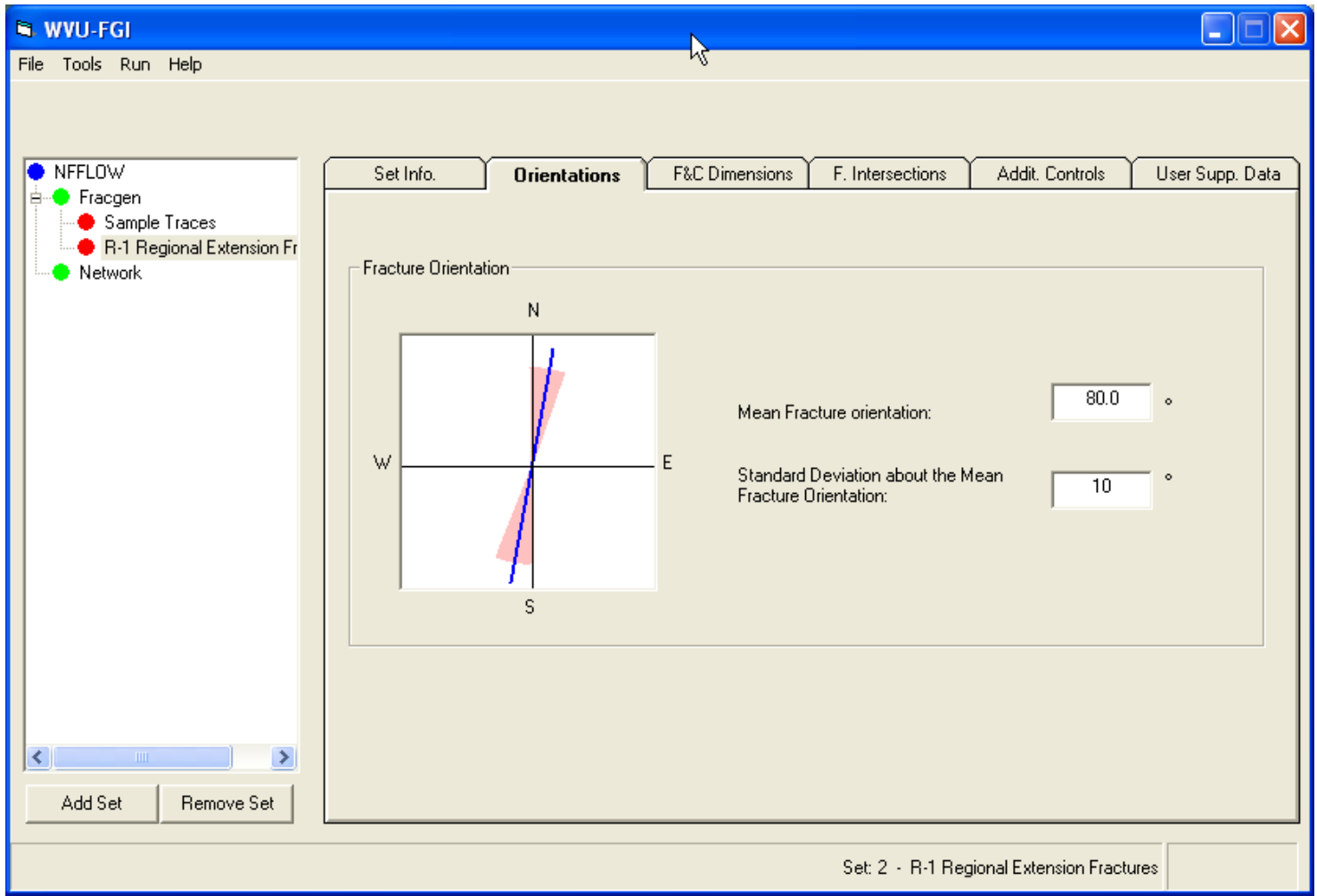

Figure 47 WVU - FGI Fracture orientation Input Screen 


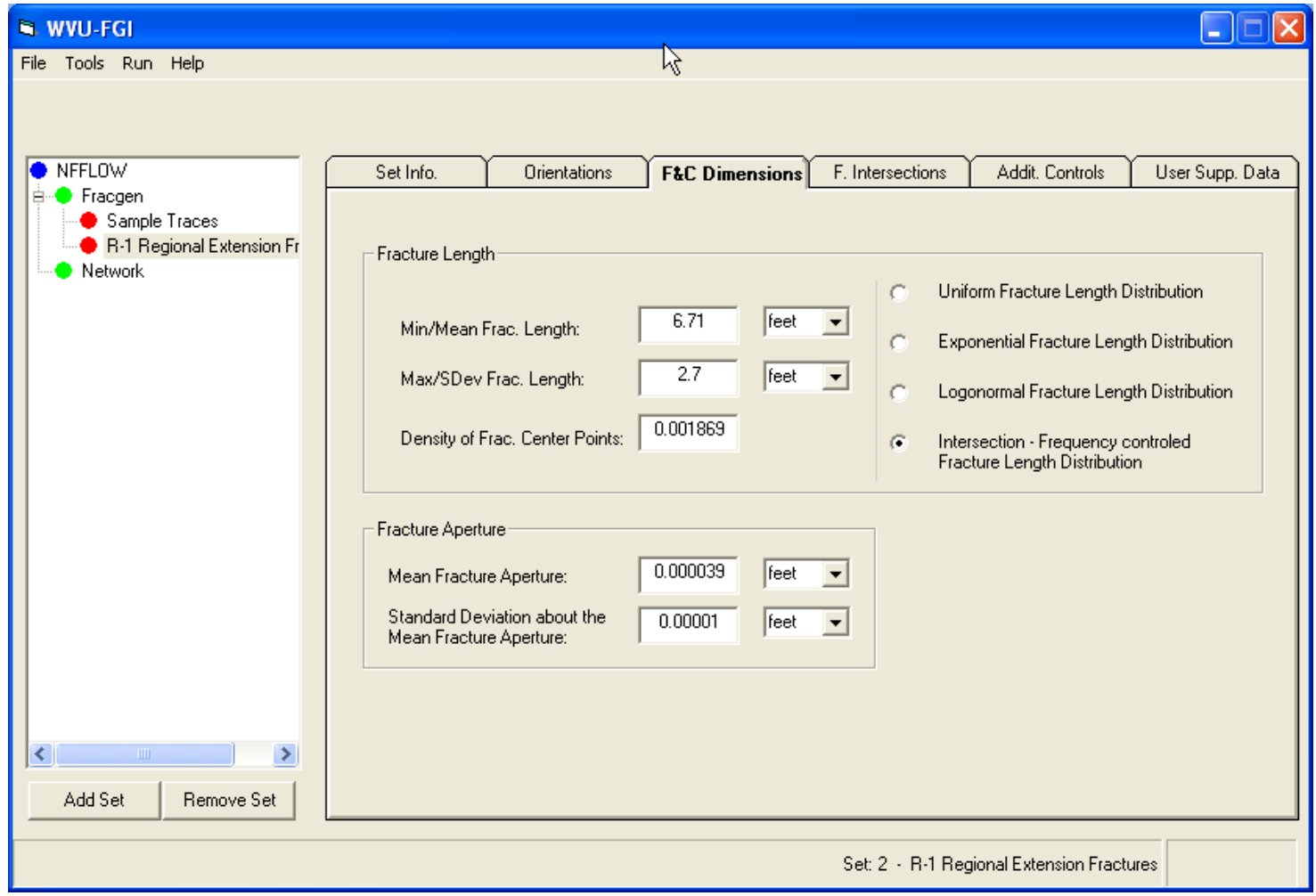

Figure 48 WVU - FGI Fracture properties - Input Screen

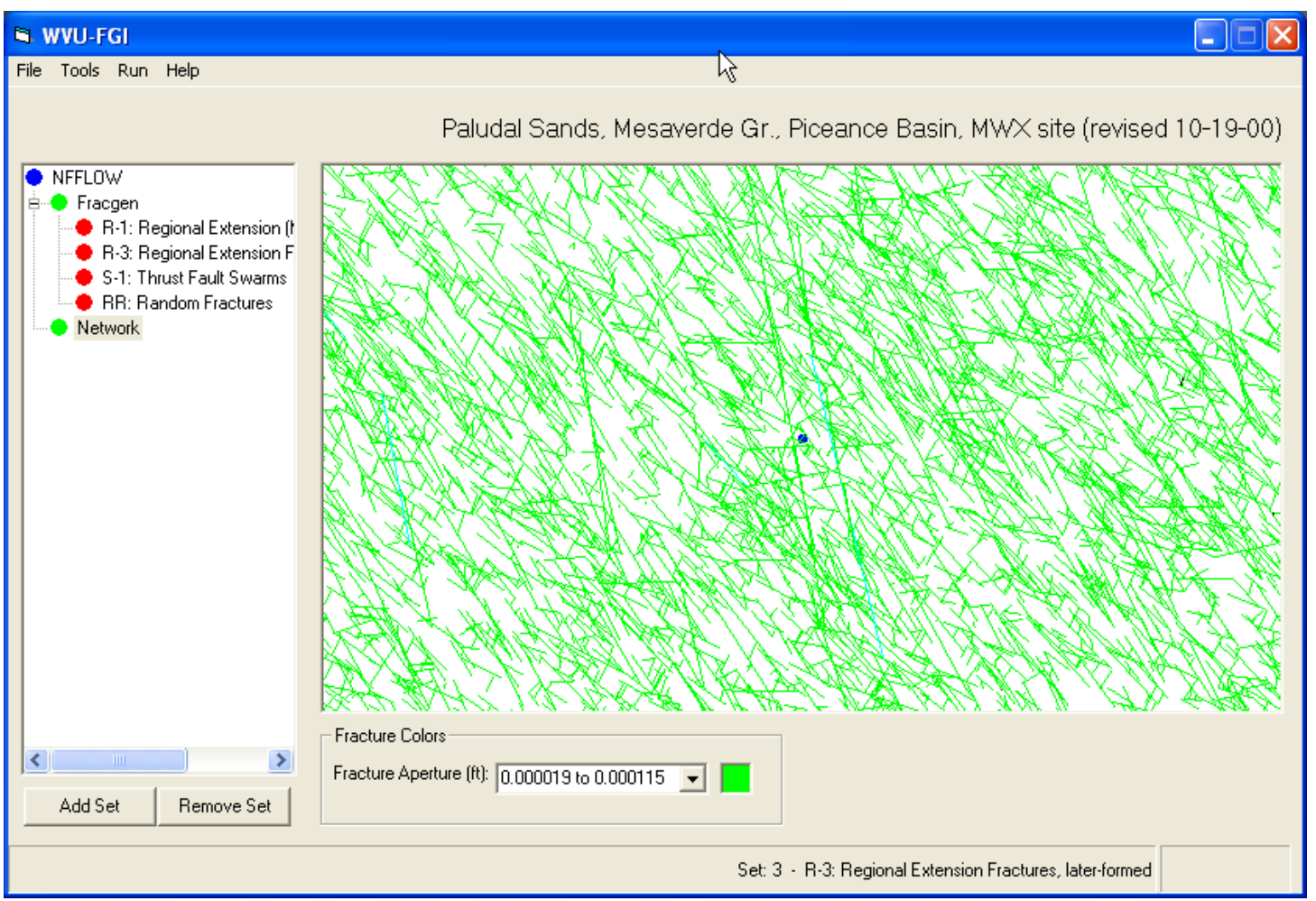

Figure 49 WVU - FGI Fracture Network Visualization Screen 


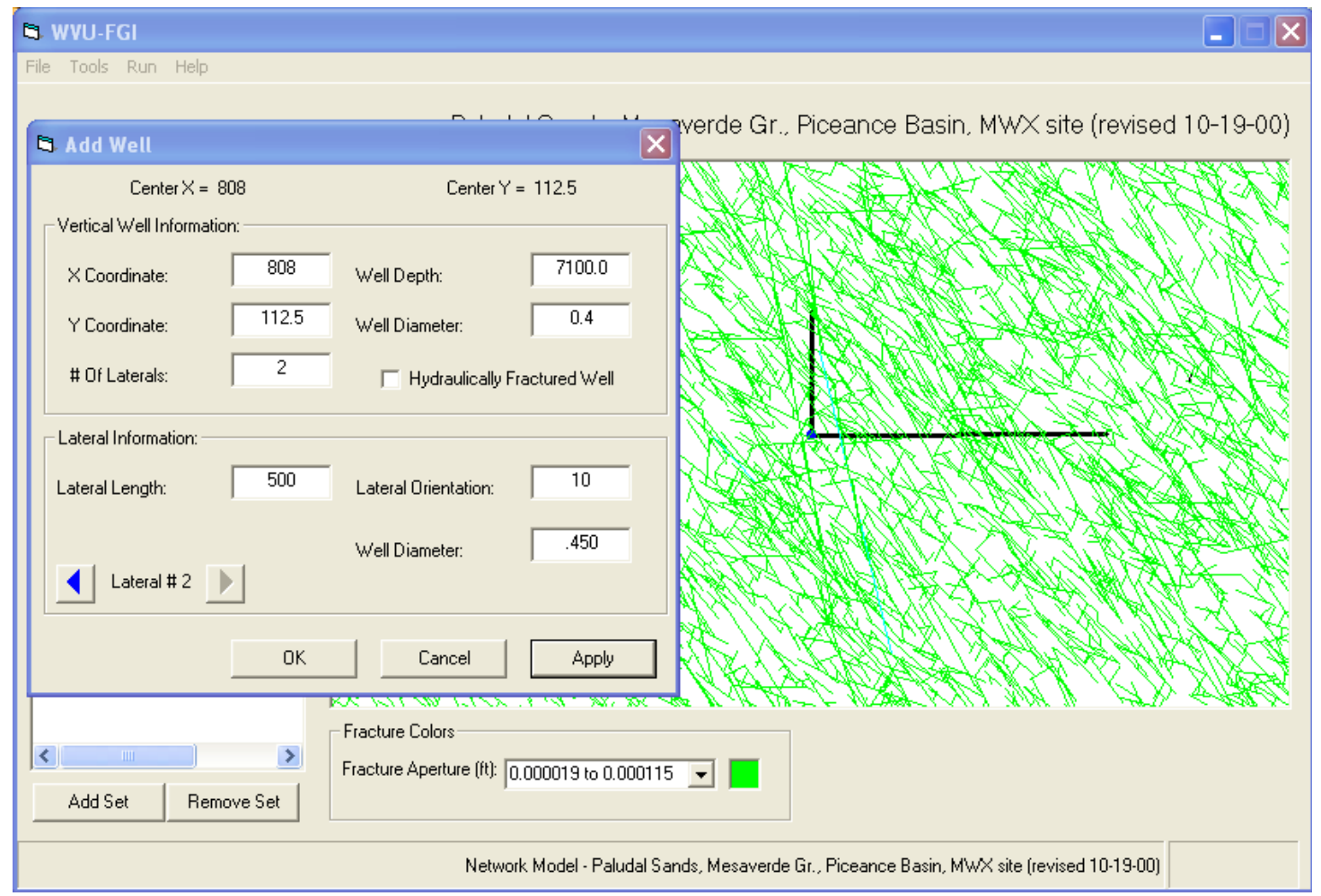

Figure 50 WVU - FGI Well Input Screen 


\section{APPENDIX - B}

Table 4 Input parameters used in Predictive Model Development

\begin{tabular}{|c|c|c|c|c|c|c|c|c|c|c|c|c|c|}
\hline Well \# & $\begin{array}{c}\text { Depth } \\
\text { (ft) }\end{array}$ & $\begin{array}{c}\text { GR } \\
\text { Respons } \\
\text { e (API ) }\end{array}$ & $\begin{array}{c}\text { HM - } \\
\text { Perm (k) }\end{array}$ & $\begin{array}{c}\text { HM - } \\
\text { Porosity }\end{array}$ & $\begin{array}{l}\text { Log - } \\
\text { Porosity }\end{array}$ & $\begin{array}{c}\text { Pay } \\
\text { thicknes } \\
\text { s (ft) }\end{array}$ & $\begin{array}{c}\text { Porosity } \\
\text { (\%) }\end{array}$ & RHOВ & $\begin{array}{c}\text { TC - } \\
\text { Drainage } \\
\text { Area } \\
\text { [Acre] }\end{array}$ & $\begin{array}{c}\text { TC - } \\
\text { Permeabi } \\
\text { lity }\end{array}$ & $\mathbf{T c}-\mathbf{X f}$ & $\begin{array}{c}\text { Total } \\
\text { Fluid } \\
\text { Injected } \\
\text { (bbl) }\end{array}$ & $\begin{array}{c}\text { Total } \\
\text { Propped } \\
\text { Interval }\end{array}$ \\
\hline 1 & 2795 & 260 & $1.5 \mathrm{E}-08$ & 3 & 12 & 127 & 12 & 2.4 & 20.538 & 0.0854161 & 94.585 & 320 & 343 \\
\hline 2 & 3330 & 357.13 & $4 \mathrm{E}-08$ & 5 & 10 & 126 & 10 & 2.6 & 21.619 & 0.14346 & 32.348 & 302.72 & 305 \\
\hline 3 & 3106 & 355 & $5 \mathrm{E}-08$ & 3 & 11 & 122 & 11 & 2.64 & 12.356 & 0.140137 & 36.682 & 302.17 & 241 \\
\hline 4 & 2956 & 327.97 & 0.0000001 & 1 & 12 & 128 & 12 & 2.63 & 6.784 & 0.1485929 & 38.828 & 302.53 & 220 \\
\hline 5 & 4058 & 385 & $4.5 \mathrm{E}-07$ & 1 & 12 & 135 & 12 & 2.55 & 35.579 & 0.2481101 & $\begin{array}{l}44.461 \\
\end{array}$ & 249.68 & 297 \\
\hline 6 & 4465 & 370 & $2 \mathrm{E}-08$ & 4 & 13 & 135 & 13 & 2.6 & 24.96 & 0.1485929 & 52.136 & 328.96 & 240 \\
\hline 7 & 3786 & 300.17 & $1.8 \mathrm{E}-08$ & 1.45 & 11 & 130 & 11 & 2.62 & 9.768 & 0.1203358 & 32.615 & 283.23 & 208 \\
\hline 8 & 3486 & 307.35 & $5 \mathrm{E}-08$ & 5 & 13 & 132 & 13 & 2.62 & 39.64 & 0.2539935 & 25.27 & 298.52 & 302 \\
\hline 9 & 3684 & 350 & $9 \mathrm{E}-10$ & 4 & 12 & 130 & 12 & 2.55 & 11.465 & 0.0243787 & 50.477 & 291.96 & 233 \\
\hline 10 & 3217 & 327.81 & $7 \mathrm{E}-09$ & 4.5 & 11 & 132 & 11 & 2.65 & 23.328 & 0.0615243 & 50.403 & 316.75 & 229 \\
\hline 11 & 3788 & 336.71 & $3 \mathrm{E}-08$ & 4 & 11 & 132 & 11 & 2.61 & 0.327 & 0.0742115 & 39.767 & 292.68 & 143 \\
\hline 12 & 3413 & 320.54 & $2 \mathrm{E}-08$ & 3.5 & 10 & 130 & 10 & 2.63 & 12.458 & 0.1257416 & 36.833 & 242.03 & 222 \\
\hline 13 & 3312 & 288.82 & 0.0000004 & 5 & 12 & 114 & 12 & 2.62 & 5.251 & 0.2992742 & 29.89 & 273.74 & 251 \\
\hline 14 & 3500 & 154.2 & $5 \mathrm{E}-09$ & 5 & 15 & 120 & 15 & 2.61 & 81.715 & 0.122829 & 188.667 & 292.27 & 321 \\
\hline 15 & 3537 & 338.55 & 0.0000004 & 3 & 18 & 128 & 18 & 2.63 & 19.929 & 0.1872867 & 116.465 & 262.07 & 208 \\
\hline 16 & 3853 & 332.55 & 1E-08 & 3 & 15 & 128 & 15 & 2.61 & 14.455 & 0.0691731 & 39.676 & 292.15 & 234 \\
\hline 17 & 3790 & 346.89 & 2E-09 & 5 & 13 & 126 & 13 & 2.6 & 13.391 & 0.0540838 & 38.188 & 309.64 & 269 \\
\hline 18 & 3100 & 332.74 & $1 \mathrm{E}-08$ & 5 & 12 & 126 & 12 & 2.59 & 39.15 & 0.1108592 & 43.53 & 296.83 & 315 \\
\hline 19 & 3046 & 354.68 & 0.0000001 & 5 & 12 & 130 & 12 & 2.62 & 20.691 & 0.2789556 & 31.646 & 291.96 & 221 \\
\hline 20 & 2865 & 336.18 & $1.3 \mathrm{E}-08$ & 5 & 11 & 130 & 11 & 2.6 & 22.167 & 0.0927179 & 32.755 & 29.16 & 552 \\
\hline 21 & 3049 & 297.32 & 4E-08 & 5 & 11 & 130 & 11 & 2.64 & 19.666 & 0.1464323 & 30.852 & 296.88 & 310 \\
\hline 22 & 3578 & 318.24 & $7.5 \mathrm{E}-09$ & 3 & 11 & 130 & 11 & 2.66 & 10.876 & 0.0669795 & 34.415 & 275.38 & 214 \\
\hline 23 & 3815 & 380 & 4E-08 & 3 & 12 & 160 & 12 & 2.65 & 10.822 & 0.2187445 & 22.886 & 299.07 & 214 \\
\hline 24 & 3180 & 320.08 & $1 \mathrm{E}-08$ & 5 & 12 & 120 & 12 & 2.62 & 9.105 & 0.0534537 & 44.983 & 28.18 & 604 \\
\hline 25 & 3157 & 320 & $8.5 \mathrm{E}-09$ & 2.7 & 11 & 127 & 11 & 2.45 & 19.132 & 0.0449691 & 152.15 & 308 & 123 \\
\hline 26 & 3128 & 336.33 & 9E-09 & 2 & 12 & 150 & 12 & 2.63 & 10.546 & 0.0786895 & 22.592 & 306.82 & 233 \\
\hline 27 & 3821 & 336.08 & $4.5 \mathrm{E}-09$ & 3 & 12 & 130 & 12 & 2.59 & 13.861 & 0.0523687 & 25.902 & 283.18 & 232 \\
\hline 28 & 3804 & 316.76 & $1.2 \mathrm{E}-08$ & 5 & 12 & 126 & 12 & 2.6 & 22.82 & \begin{tabular}{|l|}
0.095195 \\
\end{tabular} & 22.66 & 164.02 & 130 \\
\hline 29 & 3662 & 380 & $6.5 \mathrm{E}-09$ & 2.5 & 10 & 126 & 10 & 2.55 & 5.642 & 0.03475 & 24.788 & 285.24 & 232 \\
\hline 30 & 2899 & 370 & 1.32E-08 & 5 & 10 & 126 & 10 & 2.55 & 22.285 & 0.1045506 & 19.705 & 272.3 & 228 \\
\hline 31 & 3040 & 397.72 & 0.0000005 & 5 & 11 & 126 & 11 & 2.6 & 66.762 & 0.2889364 & 60.905 & 292.84 & 371 \\
\hline 32 & 4196 & 341.63 & 7.5E-09 & 5 & 12 & 126 & 12 & 2.62 & 21.475 & 0.0536105 & 43.963 & 300.71 & 233 \\
\hline 33 & 3844 & 320.8 & 5E-09 & 1.5 & 12 & 126 & 12 & 2.63 & 5.821 & 0.0377206 & 25.177 & 291.6 & 262 \\
\hline 34 & 3270 & 335.63 & 0.0000005 & 5 & 12 & 127 & 12 & 2.63 & 104.305 & 0.2181046 & 71.052 & 291.6 & 460 \\
\hline 35 & 3455 & 317.61 & $1.3 \mathrm{E}-07$ & 4 & 10 & 128 & 10 & 2.59 & 8.921 & 0.0660056 & 207.793 & 173.1 & 358 \\
\hline 36 & 3540 & 370 & 0.0000004 & 5 & 10 & 128 & 10 & 2.55 & 3.091 & 0.1131559 & 61.155 & 320.76 & 367 \\
\hline 37 & 3964 & 360 & $3 \mathrm{E}-08$ & 5 & 10 & 135 & 10 & 2.55 & 21.971 & 0.140137 & 32.61 & 292.15 & 325 \\
\hline 38 & 3465 & 390 & $8 \mathrm{E}-08$ & 4.5 & 12 & 130 & 12 & 2.634 & 25.623 & 0.7122953 & 105.647 & 256.26 & 404 \\
\hline 39 & 3630 & 336.09 & 7.5E-09 & 3.5 & 12 & 160 & 12 & 2.62 & 24.2 & 0.1161791 & 34.224 & 110 & 88 \\
\hline 40 & 3618 & 400 & $1.2 \mathrm{E}-08$ & 4 & 14 & 128 & 14 & 2.6 & 15.296 & 0.0727052 & 81.626 & 242 & 164 \\
\hline 41 & 3782 & 412.56 & $1.7 \mathrm{E}-08$ & 5 & 11 & 132 & 11 & 2.65 & 32.808 & 0.09299 & 149.432 & 284 & 183 \\
\hline 42 & 3676 & 278.79 & $7 \mathrm{E}-09$ & 5 & 12 & 160 & 12 & 2.64 & 34.947 & 0.1148256 & 24.676 & 352 & 296 \\
\hline 43 & 3757 & 391.76 & $1.2 \mathrm{E}-08$ & 2 & 13 & 128 & 13 & 2.67 & 10.927 & 0.1161791 & 22.997 & 251.87 & 203 \\
\hline 44 & 4038 & 338.34 & $1.8 \mathrm{E}-09$ & 5 & 14 & 126 & 14 & 2.62 & 18.743 & 0.0265403 & 17.376 & 222 & 197 \\
\hline 45 & 4020 & 363.33 & $6.5 \mathrm{E}-09$ & 3 & 12 & 170 & 12 & 2.63 & 14.457 & 0.0727052 & 56.682 & 255.15 & 260 \\
\hline 46 & 3642 & 308.16 & $5 \mathrm{E}-08$ & 3 & 13 & 130 & 13 & 2.63 & 17.742 & \begin{tabular}{|l|}
0.2346776 \\
\end{tabular} & 29.304 & 238.2 & 318 \\
\hline 47 & 3417 & 220.17 & $4.65 \mathrm{E}-09$ & 1.5 & 12 & 132 & 12 & 2.61 & 9.761 & 0.04658 & 32.604 & 256.79 & 198 \\
\hline 48 & 3690 & 323.28 & $3.5 \mathrm{E}-09$ & 2.5 & 12 & 160 & 12 & 2.63 & 15.026 & 0.0335496 & 57.789 & 329.87 & 224 \\
\hline 49 & 3603 & 339.68 & $2.1 \mathrm{E}-08$ & 3 & 12 & 120 & 12 & 2.59 & 14.57 & 0.1261105 & 26.555 & 291.05 & 321 \\
\hline 50 & 2692 & 313.35 & $6 \mathrm{E}-08$ & 1.5 & 13 & 128 & 13 & 2.59 & 2.648 & 0.1665776 & 16.981 & 307.8 & 212 \\
\hline 51 & 2964 & 329.86 & $1.8 \mathrm{E}-07$ & 5 & 13 & 120 & 13 & 2.6 & 30.205 & 0.2232765 & 52.139 & 278.18 & 232 \\
\hline 52 & 3293 & 362.54 & $1.7 \mathrm{E}-08$ & 2 & 13 & 128 & 13 & 2.62 & 52.195 & 0.1082913 & 150.786 & 290 & 426 \\
\hline 53 & 3054 & 348.66 & $6 \mathrm{E}-09$ & 3 & 12 & 120 & 12 & 2.59 & 14.084 & 0.0390702 & $\begin{array}{l}78.327 \\
\end{array}$ & 316 & 385 \\
\hline 54 & 2906 & 375 & 7E-09 & 4 & 12 & 128 & 12 & 2.63 & 17.566 & 0.0320134 & 145.791 & 265 & 298 \\
\hline 55 & 3349 & 415.27 & 0.0000001 & 5 & 14 & 124 & 14 & 2.61 & 18.759 & 0.2155637 & 18.832 & 291.6 & 381 \\
\hline 56 & 3472 & 375 & 0.000009 & 3 & 11 & 124 & 11 & 2.6 & 5.953 & 0.5252234 & 16.975 & 311.19 & 226 \\
\hline 57 & 2775 & 285.65 & 4E-09 & 1 & 10 & 122 & 10 & 2.58 & 0.034 & 0.0170029 & 12.886 & 300.53 & 192 \\
\hline 58 & 3378 & 321.61 & $5 E-09$ & 4 & 13 & 120 & 13 & 2.61 & 25.769 & 0.0493886 & 75.677 & 215 & 428 \\
\hline 59 & 2724 & 334.38 & $1.5 \mathrm{E}-08$ & 5 & 10 & 120 & 10 & 2.65 & 26.497 & 0.0983125 & 53.717 & 274.81 & 189 \\
\hline 60 & 3295 & 159.78 & $1 \mathrm{E}-08$ & 5 & 10 & 126 & 10 & 2.64 & 20.607 & \begin{tabular}{|l|}
0.081744 \\
\end{tabular} & 31.581 & 298.91 & 312 \\
\hline 61 & 2884 & 380 & $7 \mathrm{E}-08$ & 5 & 13 & 120 & 13 & 2.5 & 95.111 & 0.185105 & 40.709 & 291.6 & 424 \\
\hline 62 & 2600 & 265.37 & $8 \mathrm{E}-09$ & 5 & 10 & 126 & 10 & 2.62 & 23.226 & 0.0986009 & 38.687 & 274.43 & 220 \\
\hline 63 & 3711 & 395 & $2.5 \mathrm{E}-09$ & 2.7 & 13 & 127 & 13 & 2.65 & 15.569 & 0.0298399 & 58.823 & 316 & 209 \\
\hline 64 & 3181 & 350 & 0.0000005 & 1.7 & 12 & 120 & 12 & 2.61 & 46.96 & \begin{tabular}{|l|}
0.168541 \\
\end{tabular} & 178.779 & 1033.41 & 201 \\
\hline 65 & 3502 & 342.55 & $2.2 \mathrm{E}-08$ & 3 & 11 & 130 & 11 & 2.67 & 17.165 & 0.1070298 & 86.47 & 313.47 & 279 \\
\hline 66 & 3532 & 291.67 & $6 \mathrm{E}-09$ & 4.5 & 13 & 128 & 13 & 2.62 & 32.803 & 0.0908359 & 59.768 & 204 & 196 \\
\hline 67 & 3359 & 288.89 & $1 \mathrm{E}-08$ & 1.7 & 13 & 120 & 13 & 2.62 & 11.798 & 0.0735622 & 71.687 & 252 & 296 \\
\hline 68 & 3365 & 302.43 & $1.4 \mathrm{E}-09$ & 3.5 & 14 & 130 & 14 & 2.53 & 21.19 & 0.0236056 & 32.025 & 257 & 174 \\
\hline 69 & 3159 & 367.24 & 9E-09 & 3 & 11 & 125 & 11 & 2.62 & 30.89 & 0.0761942 & 82.856 & 337 & 208 \\
\hline 70 & 3316 & 400 & $4.5 \mathrm{E}-07$ & 5 & 14 & 127 & 14 & 2.5 & 9.891 & 0.2374436 & 20.512 & 294.7 & 236 \\
\hline 71 & 2668 & 338.62 & $4 \mathrm{E}-08$ & 1 & 13 & 124 & 13 & 2.59 & 4.688 & 0.1447264 & 56.484 & 257.3 & 238 \\
\hline 72 & 3037 & 337.17 & 0.0000003 & 4.8 & 12 & 128 & 12 & 2.62 & 10.416 & 0.4058682 & 30.618 & 255.15 & 177 \\
\hline 73 & 3774 & 390 & 0.0000001 & 4.5 & 12 & 130 & 12 & 2.65 & 25.562 & 0.2539933 & 37.686 & 161.29 & 263 \\
\hline 74 & 3195 & 345.83 & $1.5 \mathrm{E}-07$ & 5 & 12 & 124 & 12 & 2.61 & 60.407 & 0.2319437 & 40.553 & 247.86 & 274 \\
\hline 75 & 3188 & 321.9 & $4.9 \mathrm{E}-09$ & 5 & 11 & 130 & 11 & 2.63 & 88.593 & 0.2161962 & 109.137 & 325.5 & 468 \\
\hline
\end{tabular}



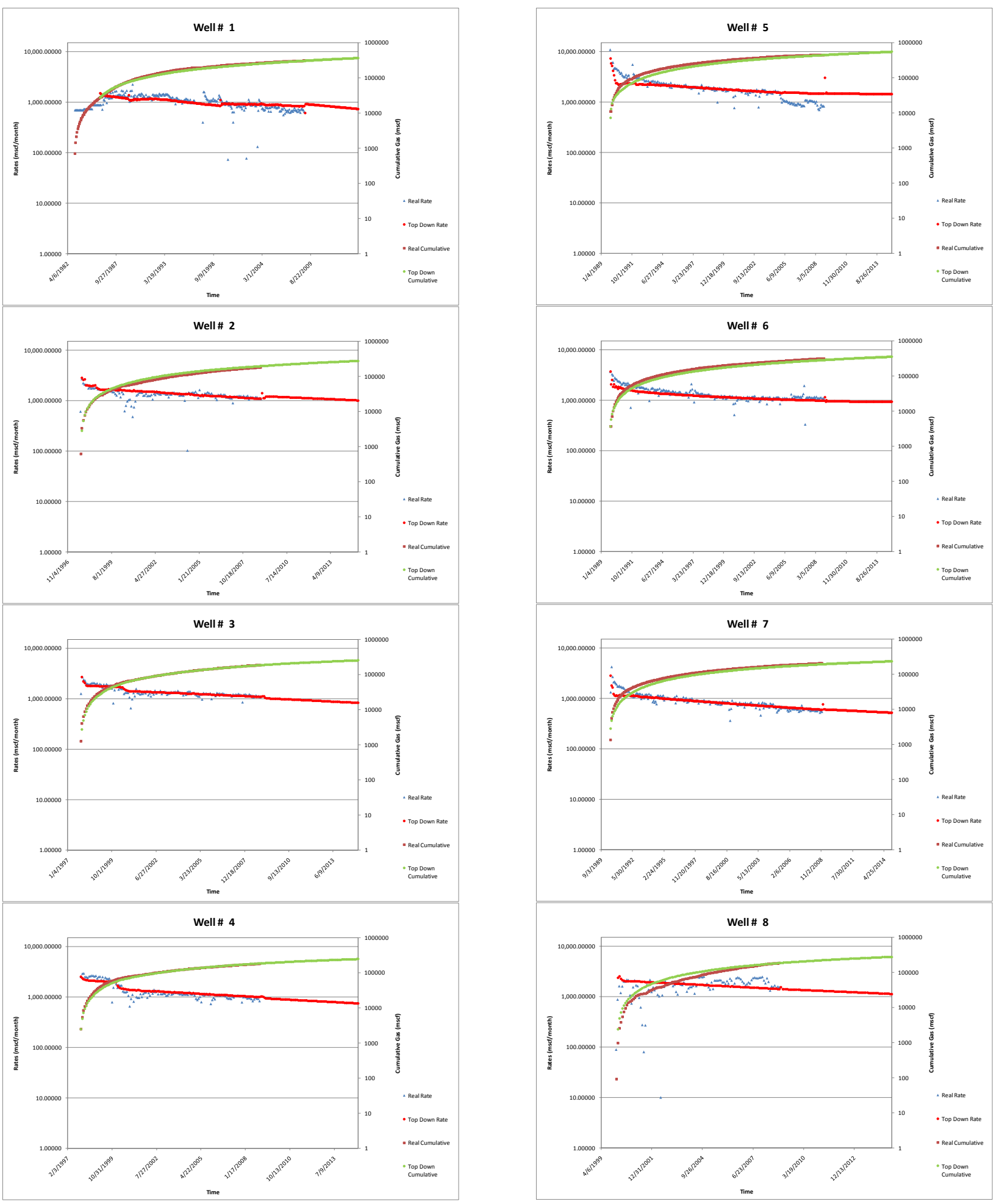

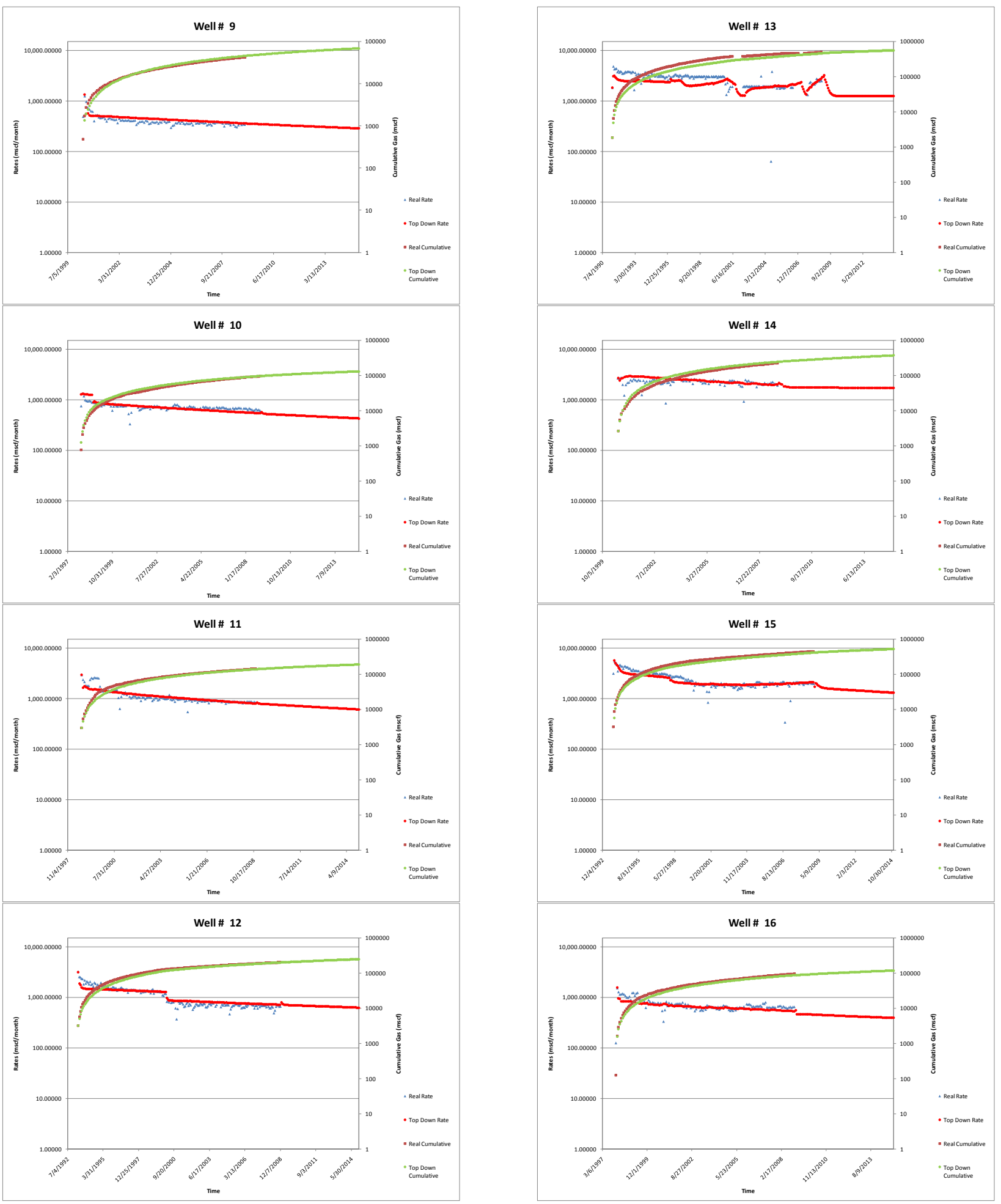

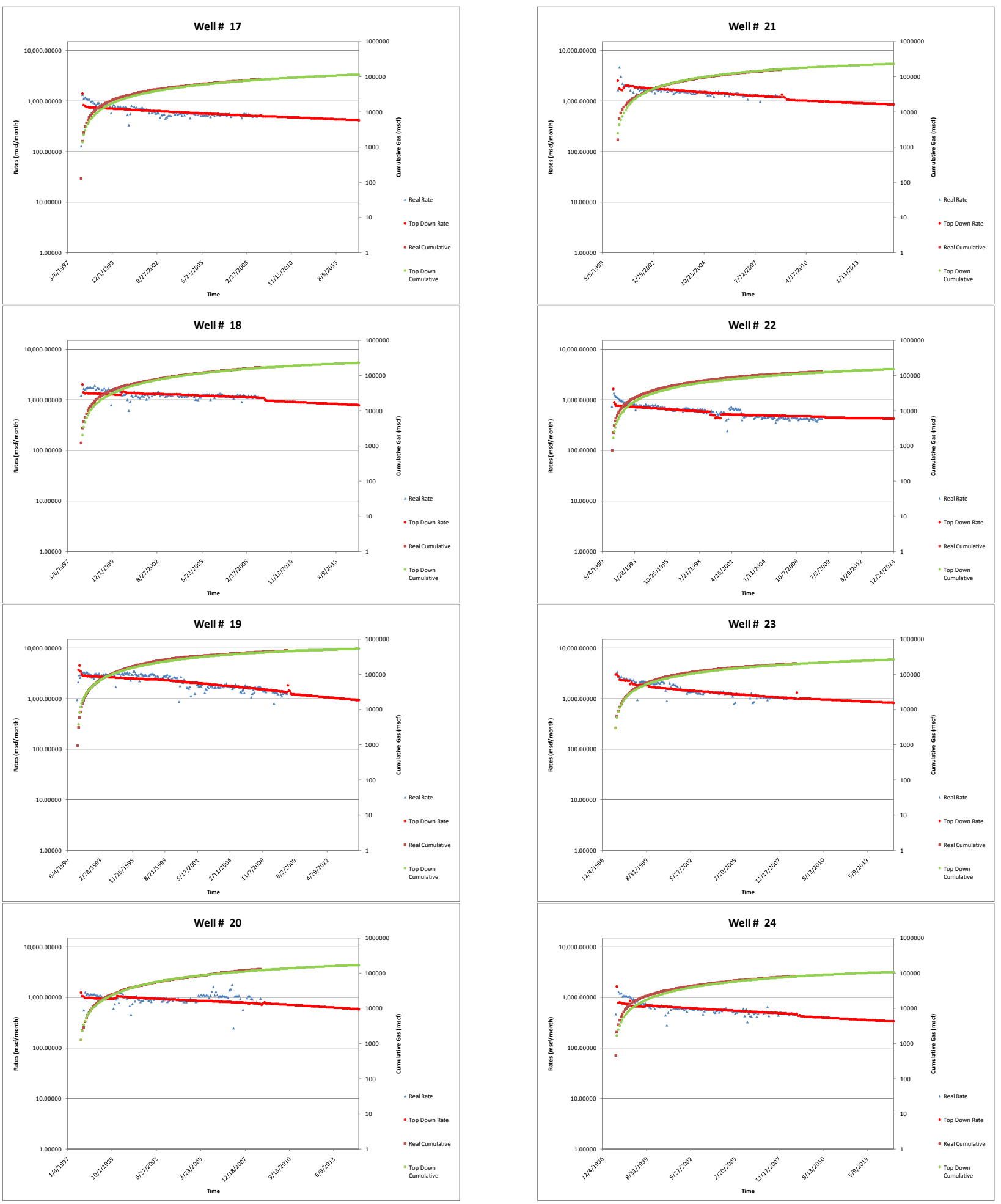

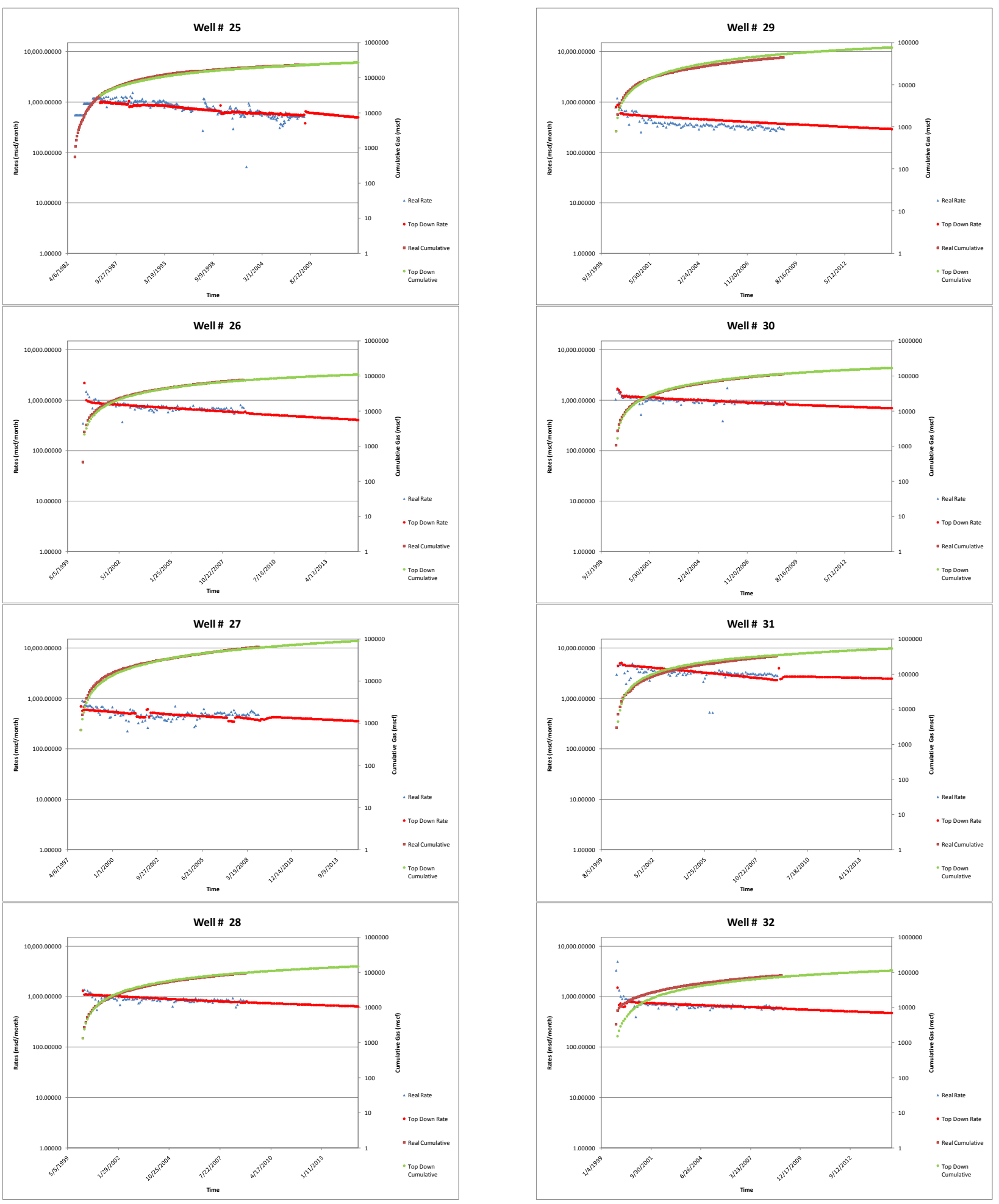

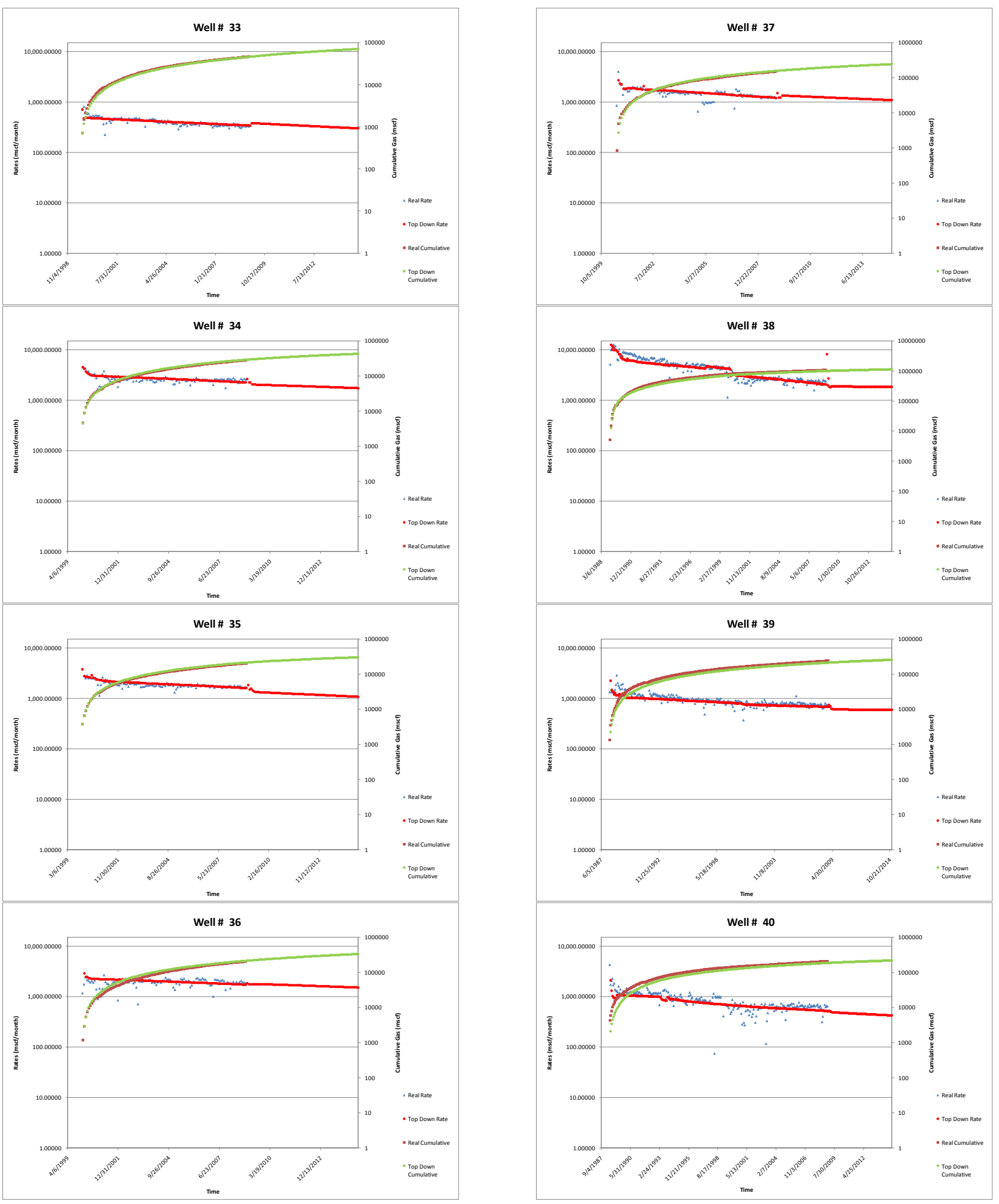

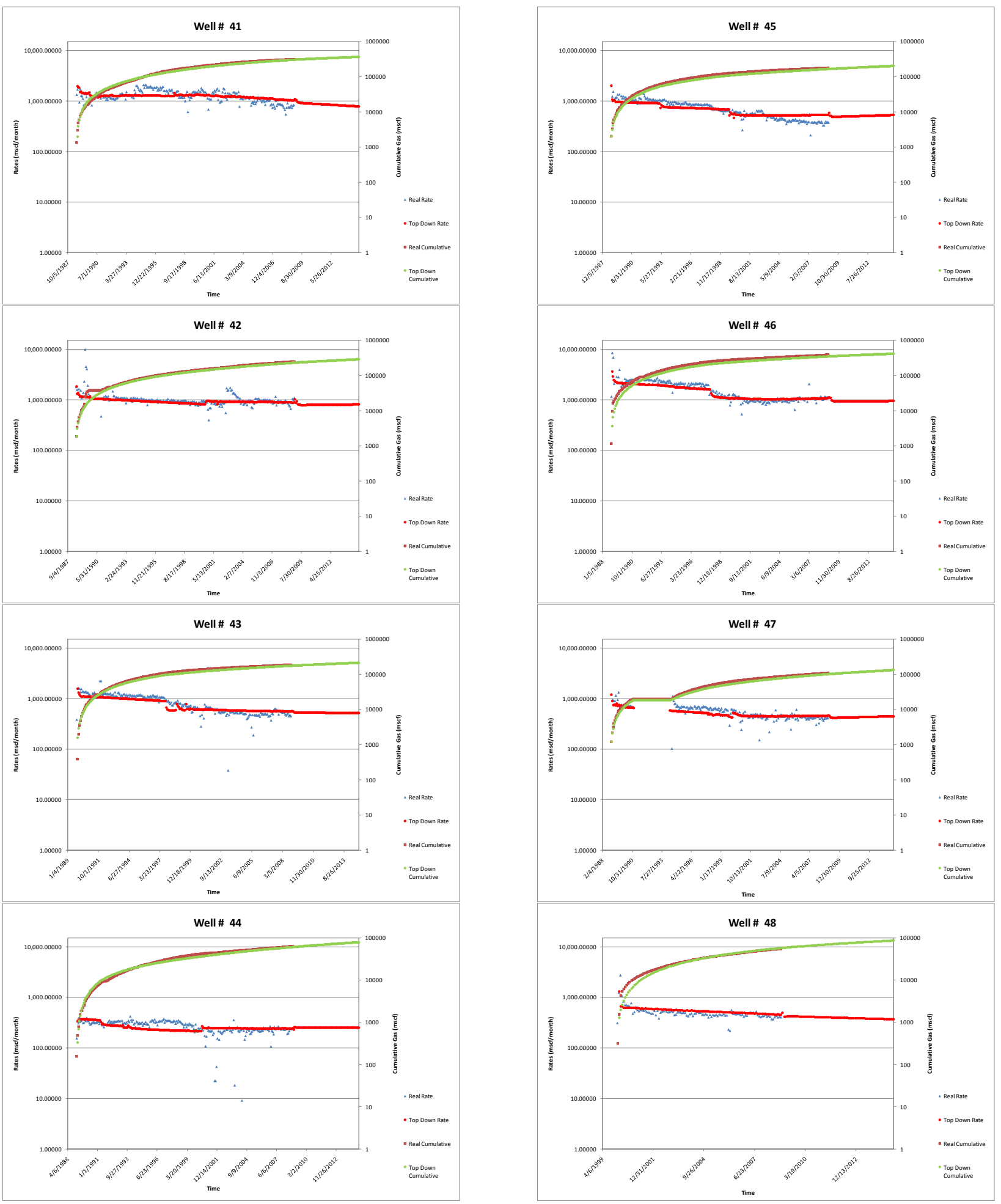

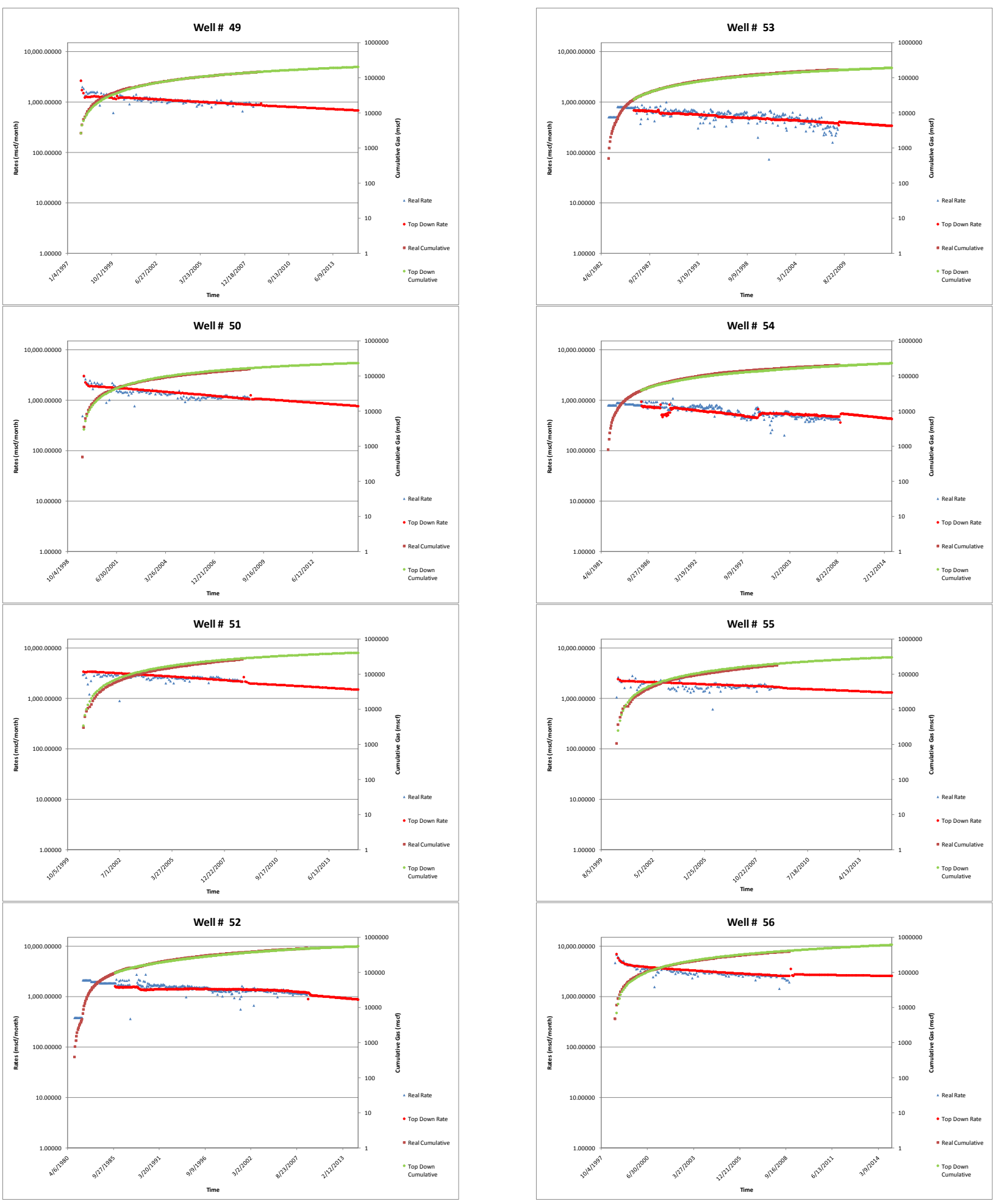

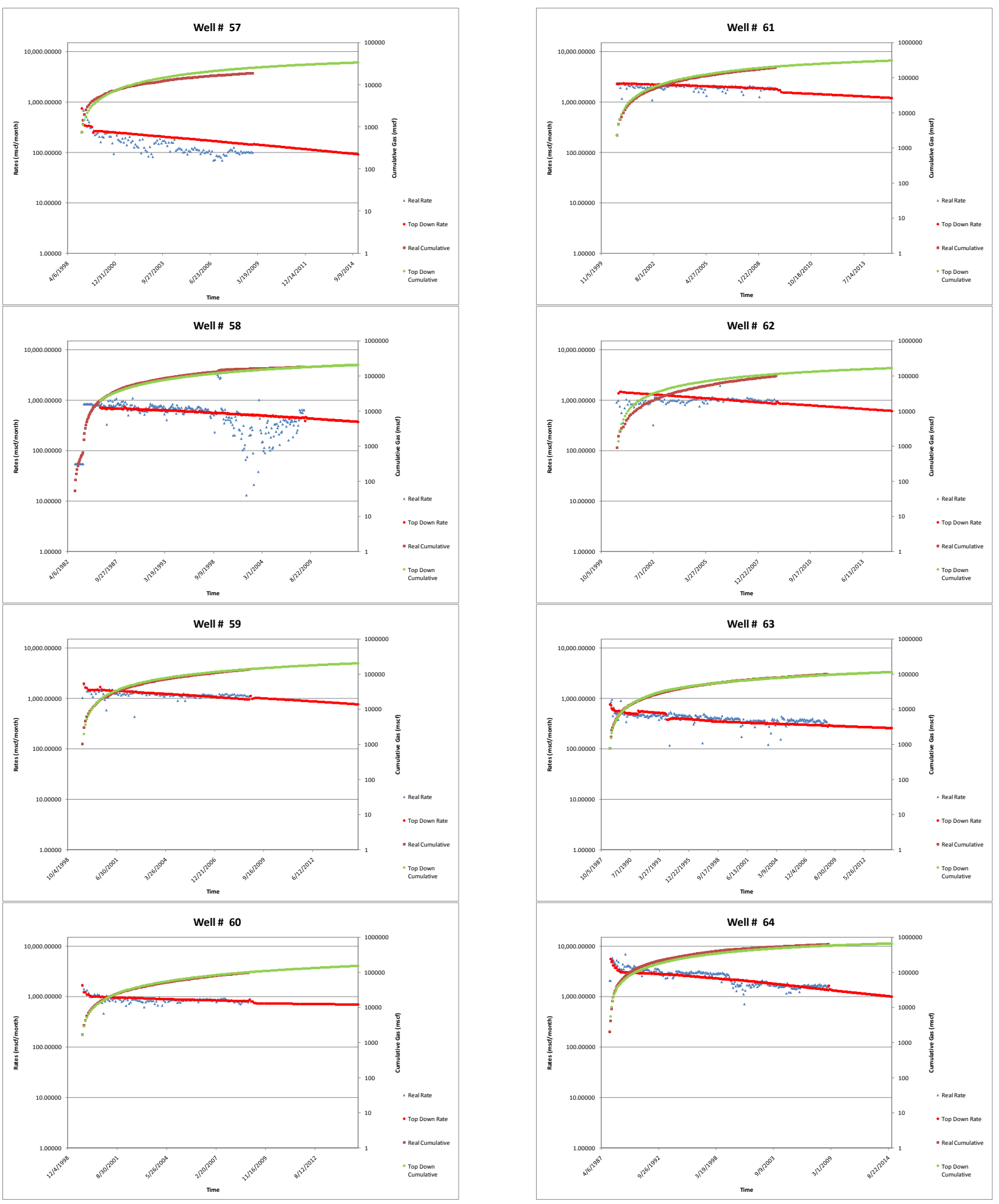

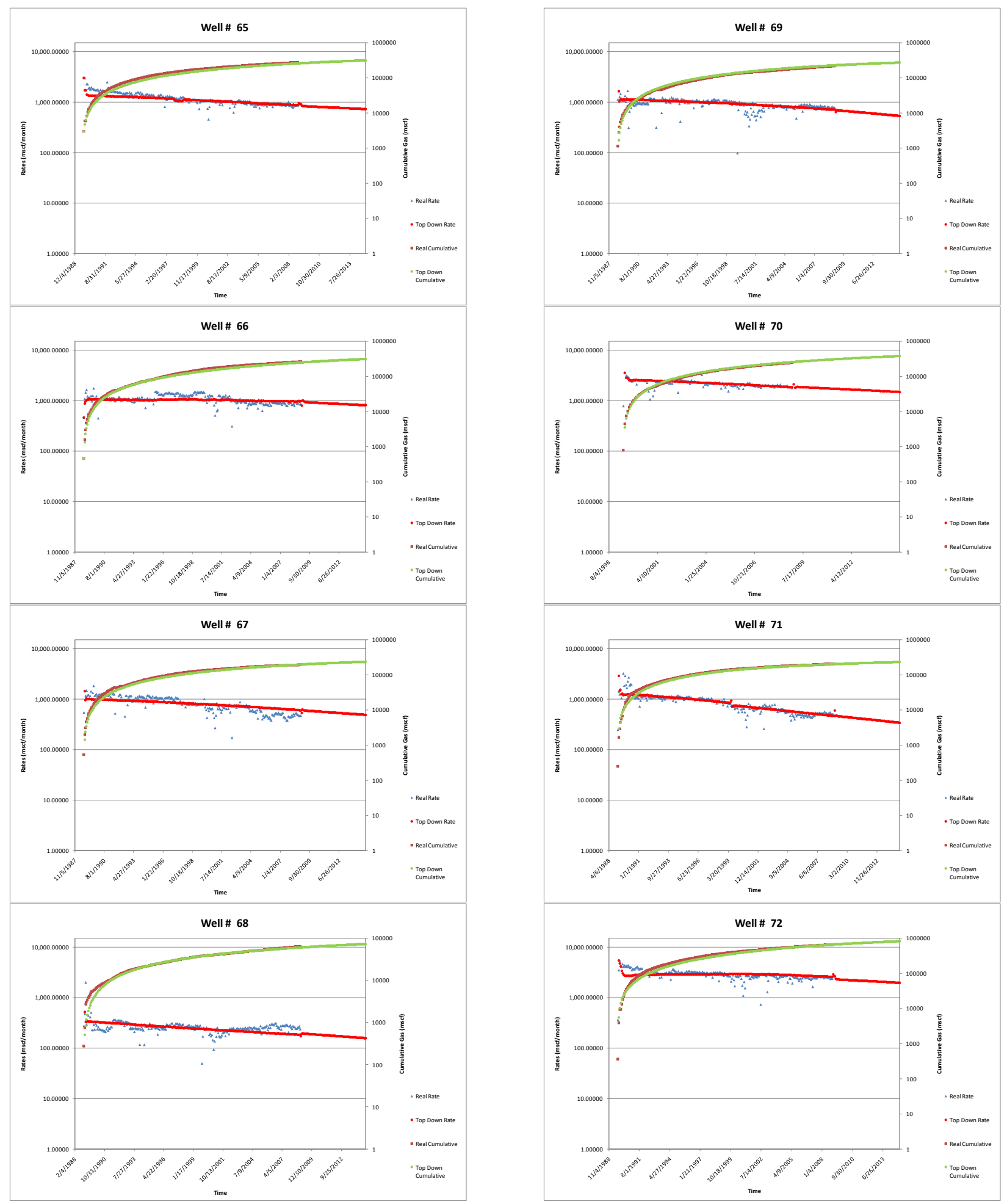

75 


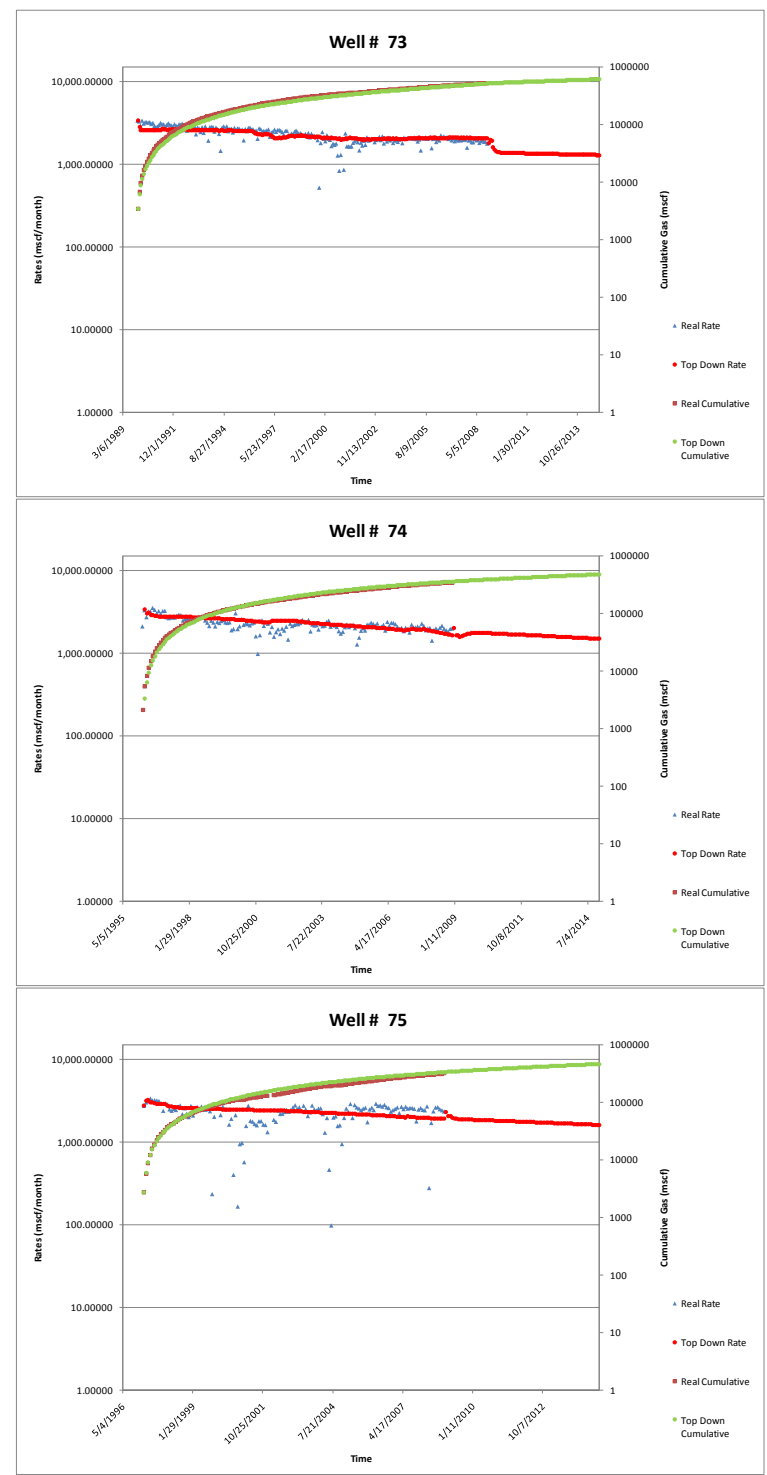

John H.

Virginia University Libraries,

Hagen

Date: 2011.05.05 09:12:58 -04'00' 